\title{
COSMIC APPROXIMATE LIMITS AND FIXED POINTS
}

\author{
J. SEGAL AND T. WATANABE \\ Dedicated to Professor Yukihiro Kodama on his sixtieth birthday
}

\begin{abstract}
We define a condition for approximate inverse systems which implies that the limit of the system has the fixed point property. Moreover, this condition is categorical in the approximate shape category. We investigate the class of complex projective $n$-space like continua with respect to the fixed point property by means of this condition. As a further application we show that the hyperspace $C(X)$ of nonempty subcontinua of an arc-like or circle-like Hausdorff continuum $X$ has the fixed point property. We also prove that $2^{X}$ and $C(X)$ have the fixed point property for any locally connected Hausdorff continuum $X$.
\end{abstract}

\section{INTRODUCTION}

Recently Mardešić and Rubin [MR] defined a generalization of the classical notion of (commutative) inverse limits called approximate limits. In addition, Mardešić and Segal [MS1, MS2, MS3], Mardešić and Watanabe [MW], and Watanabe [W1, W2, W3] have recently dealt with the idea of nearly commutative inverse systems. Since the classical notion of inverse limits has some defects even for nonmetric compacta, it was necessary to introduce this new notion of approximate limits. Approximate limits have proved to be especially useful in attacking problems in dimension theory. In this paper we use the approximate limit approach to obtain results about fixed points.

We define a cosmicality condition for approximate systems which implies that the limit of the system has the fixed point property. Moreover, this property is categorical in the approximate shape category. The significance of the latter statement is that as far as this cosmicality condition is concerned it does not matter which representation as an inverse limit of polyhedra of a Hausdorff continuum is used. As an application we show that the hyperspace $C(X)$ of all nonempty subcontinua of an arc-like or circle-like Hausdorff continuum $X$ has the fixed point property. Such results had been previously obtained only in the metric case for $C(X)$ (see [N1] or [S2]). We also show that a map $f: X \rightarrow X$ has a fixed point if and only if its approximate resolution $\mathbf{f}:(\mathscr{X}, \mathscr{U}) \rightarrow(\mathscr{X}, \mathscr{U})$

Received by the editors May 30,1990. This paper was presented on September 3, 1990 to the General Topology and Geometric Topology Symposium at the University of Tsukuba, Tsukuba, Japan.

1991 Mathematics Subject Classification. Primary 54B25, 54H25, 55M20, 54C56, 54B20.

Key words and phrases. Approximate inverse system, coincidence point, fixed point, hyperspace, cosmic, shape. 
has an approximate fixed point (see (3.2)). Further, we prove that $2^{X}$ and $C(X)$ have the fixed point property for any locally connected Hausdorff continuum $X$. This was previously known in the metric case since in that case both hyperspaces are absolute retracts. However, in the nonmetric (locally connected) case the hyperspace $C(X)$ may not even be contractible (see Example (16.41) of [N1]).

In $\S 1$ we summarize the needed properties of approximate limits and resolutions. In $\S 2$ we investigate the approximate nearness of system maps. In $\S 3$ we consider approximate coincidence points. In $\S 4$ we define and investigate the notion of cosmicality. In $\S 5$ we define the notion of cosmicality for approximate inverse systems and approximate resolutions. We prove that if such a system has this property then its limit space has the fixed point property. Further we generalize the notion of cosmicality to maps of approximate systems. We also exhibit an example of a continuum with the fixed point property, but which does not satisfy the cosmicality condition. In $\S 6$ we investigate the class of complex projective $n$-space like continua with respect to the fixed point property by means of the cosmicality condition. In $\S 7$ we consider nearly extendable maps and approximate resolutions. In $\S 8$ we consider refinable maps and cosmicality. In $\S 9$ we investigate the functorial properties of hyperspaces and then prove that if an approximate resolution for a Hausdorff continuum $X$ induces a cosmic approximate ANR-resolution for $2^{X}$ (or $\left.C(X)\right)$, then $2^{X} \quad(C(X))$ has the fixed point property. As a corollary we have that if $X$ is an arc-like or circle-like Hausdorff continuum, then $C(X)$ has the fixed point property. As another corollary we have if $X$ is a locally connected Hausdorff continuum, then $2^{X}$ and $C(X)$ have the fixed point property.

LOCATION OF SPECIAL NOTATION

\begin{tabular}{|llllll|}
\hline (A1) & 1.1 & $(\mathrm{AN} 1)$ & 2.2 & $(\mathrm{NE})$ & {$[\mathrm{W} 3]$} \\
$(\mathrm{A} 2)$ & 1.1 & $(\mathrm{AN} 2)$ & 2.3 & $(\mathrm{NE})^{*}$ & 7.0 \\
$(\mathrm{~A} 3)$ & 1.1 & $(\mathrm{AS})$ & 1.7 & $(\mathrm{R} 1)$ & 1.10 \\
$(\mathrm{AC})$ & 5.1 & $(\mathrm{AU})$ & 1.2 & $(\mathrm{R} 2)$ & 1.10 \\
$(\mathrm{AC} 1)$ & 5.1 & $(\mathrm{API})$ & 8.0 & $(\mathrm{RF} 1)$ & 1.14 \\
$(\mathrm{AFP})$ & 3.2 & $(\mathrm{API} 1)$ & 8.5 & $(\mathrm{RF} 1)^{\prime}$ & 1.15 \\
$(\mathrm{AI} 3)$ & {$[\mathrm{W} 1]$} & $(\mathrm{B} 1)$ & 1.11 & $(\mathrm{SF})$ & 1.15 \\
$(\mathrm{AM})$ & 1.10 & $(\mathrm{~B} 2)$ & 1.11 & $(\mathrm{U})$ & 1.15 \\
$(\mathrm{AM} 2)$ & {$[\mathrm{W} 1]$} & $(\mathrm{C})$ & 1.3 & $(\mathrm{UL})$ & 1.8 \\
$(\mathrm{AMC})$ & 4.0 & $(\mathrm{CS})$ & 1.7 & $(\mathrm{US})$ & 5.8 \\
$(\mathrm{AMU})$ & 1.10 & $(\mathrm{E} 1)$ & {$[\mathrm{MS}]$} & $(*)$ & 1.15 \\
$(\mathrm{AN})$ & 2.0 & $(\mathrm{E} 2)$ & {$[\mathrm{MS}]$} & $(* *)$ & 7.8 \\
\hline
\end{tabular}




\section{APPROXimate SySTEMS AND RESOlutions}

In this section we summarize the needed properties of approximate resolutions. Without any specification we use the same notation and terminology as in [MW]. In the following we use normal (open) coverings. Recall that these coverings coincide with numerable coverings, i.e., open coverings which admit a subordinate partition of unity. Every normal covering $\mathscr{U}$ of a space $X$ admits a normal covering $\mathscr{U}^{\prime}$ of $X$ such that the star st $\mathscr{U}^{\prime}=\left\{\operatorname{st}\left(U^{\prime}, \mathscr{U}^{\prime}\right): U^{\prime} \in \mathscr{U}^{\prime}\right\}$ refines $\mathscr{U}$. Here, for any subset $X_{0}$ of $X$, st $\left(X_{0}, \mathscr{U}^{\prime}\right)=\bigcup\left\{U^{\prime} \in \mathscr{U}^{\prime}: U^{\prime} \cap X_{0} \neq\right.$ $\varnothing\}$. Inductively, $\mathrm{st}^{0} \mathscr{U}^{\prime}=\mathscr{U}^{\prime}, \mathrm{st}^{1} \mathscr{U}^{\prime}=\mathrm{st} \mathscr{U}^{\prime}$ and $\mathrm{st}^{n} \mathscr{U}^{\prime}=\mathrm{st}\left(\mathrm{st}^{n-1} \mathscr{U}^{\prime}\right)$ for each positive integer $n$. We denote the set of all normal coverings of $X$ by $\operatorname{Cov}(X)$. If $\mathscr{U} \in \operatorname{Cov}(X)$, then also st $\mathscr{U} \in \operatorname{Cov}(X)$. If $\mathscr{U}, \mathscr{V} \in \operatorname{Cov}(X)$ and $\mathscr{V}$ refines $\mathscr{U}$, we write $\mathscr{V}<\mathscr{U}$. For $\mathscr{U}, \mathscr{V} \in \operatorname{Cov}(X)$, put $\mathscr{U} \wedge \mathscr{V}=\{U \cap V$ : $U \in \mathscr{U}$ and $V \in \mathscr{V}\} \in \operatorname{Cov}(X)$. If $f, g: Y \rightarrow X$ are $\mathscr{U}$-near mappings, i.e., if for any $y \in Y$ there exists a $U \in \mathscr{U}$ with $f(y), g(y) \in U$, we write $(f, g)<\mathscr{U}$.

(1.1) Definition. An approximate inverse system (shorter, an approximate system) $\mathscr{X}=\left\{X_{a}, \mathscr{U}_{a}, p_{a a^{\prime}}, A\right\}$ consists of the following data. A preordered set $A=(A,<)$ (we do not assume antisymmetry), which is directed and unbounded (no maximal element), for each $a \in A$, a topological space $X_{a}$ and a normal covering $\mathscr{U}_{a}$ of $X_{a}$ (called the mesh of $X_{a}$ ), and for $a<a^{\prime}$, a (continuous) mapping $p_{a a^{\prime}}: X_{a^{\prime}} \rightarrow X_{a}\left(p_{a a}=1_{X_{a}}\right.$ is the identity mapping on $X_{a}$ ). We require three conditions:

(A1) $\left(p_{a a^{\prime}} p_{a^{\prime} a^{\prime \prime}}, p_{a a^{\prime \prime}}\right)<\mathscr{U}_{a}$ for $a<a^{\prime}<a^{\prime \prime}$.

(A2) For each $a \in A$ and each $\mathscr{U} \in \operatorname{Cov}\left(X_{a}\right)$, there exists an $a^{\prime}>a$ such that $\left(p_{a a_{1}} p_{a_{1} a_{2}}, p_{a a_{2}}\right)<\mathscr{U}$, whenever $a_{2}>a_{1}>a^{\prime}$.

(A3) For each $a \in A$ and each $\mathscr{U} \in \operatorname{Cov}\left(X_{a}\right)$, there exists an $a^{\prime}>a$ such that $\mathscr{U}_{a^{\prime \prime}}<p_{a a^{\prime \prime}}^{-1} \mathscr{U}=\left\{p_{a a^{\prime \prime}}^{-1} U: U \in \mathscr{U}\right\}$, whenever $a^{\prime \prime}>a^{\prime}$.

If all the spaces $X_{a}$ belong to a class $\mathscr{C}$ of spaces, we speak of an approximate inverse system in $\mathscr{C}$ or an approximate $\mathscr{C}$-system. Sometimes we write $(\mathscr{X}, \mathscr{U})$ to emphasize the meshes $\mathscr{U}_{a}, a \in A$.

(1.2) Definition. An approximate system $\mathscr{X}$ is said to be uniform provided it satisfies the additional condition:

$$
\mathscr{U}_{a^{\prime}}<p_{a a^{\prime}}^{-1} \mathscr{U}_{a} \quad \text { for } a<a^{\prime} .
$$

(1.3) Definition. An approximate system $\mathscr{X}$ is said to be commutative provided it satisfies the commutativity condition:

$$
p_{a a^{\prime}} p_{a^{\prime} a^{\prime \prime}}=p_{a a^{\prime \prime}} \quad \text { for } a<a^{\prime}<a^{\prime \prime} .
$$

(1.4) Remark. Uniform commutative approximate systems over cofinite index sets coincide with approximate inverse systems of Watanabe [W1-W3].

(1.5) Definition. By deleting the meshes $\mathscr{U}_{a}$ in an approximate system $\mathscr{X}=$ $\left\{X_{a}, \mathscr{U}_{a}, p_{a a^{\prime}}, A\right\}$, we obtain a system $\mathbb{X}=\left\{X_{a}, p_{a a^{\prime}}, A\right\}$, which we also call an approximate system. We say that $\mathscr{X}$ and $\mathbb{X}$ are associated with each other. We distinguish the two notions by using script and blackboard boldface characters respectively. By definition, $\mathscr{X}$ determines $\mathbb{X}$. Conversely, every $\mathbb{X}$ admits 
an associated $\mathscr{X}$, which is not unique. In our statements and arguments we shall often switch from $\mathscr{X}$ to $\mathbb{X}$ and from $\mathbb{X}$ to $\mathscr{X}$.

(1.6) Remark. Let $\mathbb{X}=\left\{X_{a}, p_{a a^{\prime}}, A\right\}$ be an inverse system with an unbounded indey.ing set $A$ in the usual sense. By the Mardešić trick (see [MS, Theorem 2, p.10]), we can assume that $A$ is cofinite. If each $X_{a}$ is a metric compactum and if the directed set $A$ is cofinite and infinite, then $\mathbb{X}$ is an approximate system (see [W1, Proposition (3.8)]).

For approximate systems of metric compacta Mardešić and Rubin [MR] have defined limits. In [MW] they generalize these results to approximate systems of arbitrary spaces.

(1.7) Definition. An approximate map $\mathbf{p}=\left\{p_{a}: a \in A\right\}: X \rightarrow \mathbb{X}$ into an approximate system $\mathbb{X}=\left\{X_{a}, p_{a a^{\prime}}, A\right\}$ is a collection of maps $p_{a}: X \rightarrow X_{a}$, $a \in A$, such that the following condition holds:

(AS) For any $a \in A$ and any $\mathscr{U} \in \operatorname{Cov}\left(X_{a}\right)$ there exists an $a^{\prime}>a$ such that $\left(p_{a a^{\prime \prime}} p_{a^{\prime \prime}}, p_{a}\right)<\mathscr{U}$ for $a^{\prime \prime}>a^{\prime}$.

$\mathbf{p}: X \rightarrow \mathbb{X}$ is called commutative provided $\mathbb{X}$ is commutative and

$$
p_{a a^{\prime}} p_{a^{\prime}}=p_{a} \quad \text { for } a^{\prime}>a \text {. }
$$

(1.8) Definition. Let $\mathbb{X}=\left\{X_{a}, p_{a a^{\prime}}, A\right\}$ be an approximate system and let $\mathbf{p}=\left\{p_{a}: a \in A\right\}: X \rightarrow \mathbb{X}$ be an approximate map. We say that $\mathbf{p}$ is a limit of $\mathbb{X}$ provided it has the following universal property:

(UL) For any approximate map $\mathbf{q}=\left\{q_{a}: a \in A\right\}: Y \rightarrow \mathbb{X}$ of a space $Y$ there exists a unique map $g: Y \rightarrow X$ such that $p_{a} g=q_{a}$ for any $a \in A$.

(1.9) Remark. If $\mathbf{p}: X \rightarrow \mathbb{X}$ is a limit of $\mathbb{X}$, then the space $X$ is determined up to a unique homeomorphism. Therefore, we often speak of the limit $X$ of $\mathbb{X}$ and we write $X=\lim \mathbb{X}$.

(1.10) Definition. Let $\mathscr{X}=\left\{X_{a}, \mathscr{U}_{a}, p_{a a^{\prime}}, A\right\}$ and $\mathscr{Y}=\left\{Y_{b}, \mathscr{V}_{b}, q_{b b^{\prime}}, B\right\}$ be approximate inverse systems. An approximate map $\mathbf{f}=\left\{f, f_{b}: b \in B\right\}$ : $\mathscr{X} \rightarrow \mathscr{Y}$ consists of a function $f: B \rightarrow A$ and of maps $f_{b}: X_{f(b)} \rightarrow Y_{b}, b \in B$, such that the following condition is satisfied:

(AM) Whenever $b, b^{\prime} \in B, b<b^{\prime}$, there exists an $a \in A, a>f(b), f\left(b^{\prime}\right)$, such that $\left(q_{b b^{\prime}} f_{b^{\prime}} p_{f\left(b^{\prime}\right), a^{\prime}}, f_{b} p_{f(b), a^{\prime}}\right)<\mathrm{st} \mathscr{V}_{b}$ for any $a^{\prime}>a$.

The approximate map $\mathbf{f}$ is called uniform provided $\mathbf{f}$ satisfies the additional condition

(AMU)

$$
\mathscr{U}_{f(b)}<f_{b}^{-1} \mathscr{V}_{b} \quad \text { for } b \in B
$$

We will now define the basic notion of approximate resolution of a space.

Let $\mathscr{P}$ be a collection of spaces. For an approximate map $\mathbf{p}=\left\{p_{a}: a \in A\right\}$ : $X \rightarrow \mathscr{X}$ into an approximate system $\mathscr{X}=\left\{X_{a}, \mathscr{U}_{a}, p_{a a^{\prime}}, A\right\}$ we consider the following conditions:

(R1) For any $P \in \mathscr{P}, \mathscr{V} \in \operatorname{Cov}(P)$, and mapping $f: X \rightarrow P$ there is an $a \in A$ such that for every $a^{\prime}>a$ there exists a mapping $g: X_{a^{\prime}} \rightarrow P$ satisfying $\left(g p_{a^{\prime}}, f\right)<\mathscr{V}$.

(R2) For every $P \in \mathscr{P}$ and $\mathscr{V} \in \operatorname{Cov}(P)$ there is a $\mathscr{V}^{\prime} \in \operatorname{Cov}(P)$ such that for any $a \in A$ and for any two maps $g, g^{\prime}: X_{a} \rightarrow P$, for which $\left(g p_{a}, g^{\prime} p_{a}\right)<$ $\mathscr{V}^{\prime}$, there exists an $a^{\prime}>a$ such that $\left(g p_{a a^{\prime \prime}}, g^{\prime} p_{a a^{\prime \prime}}\right)<\mathscr{V}$ for any $a^{\prime \prime}>a^{\prime}$. 
(1.11) Definition. An approximate resolution of a space $X$ is an approximate map $\mathbf{p}=\left\{p_{a}: a \in A\right\}: X \rightarrow \mathscr{X}$ of $X$ into an approximate system $\mathscr{X}=$ $\left\{X_{a}, \mathscr{U}_{a}, p_{a a^{\prime}}, A\right\}$, which satisfies conditions (R1) and (R2) for the collection $\mathscr{P}=$ POL of all polyhedra (endowed with the $\mathrm{CW}$ topology). If $\mathscr{C}$ is a class of spaces and all $X_{a}, a \in A$, belong to $\mathscr{C}$, we speak of an approximate $\mathscr{C}$ resolution.

Approximate resolutions can also be characterized by conditions of a different nature, which are usually easier to verify than conditions (R1) and (R2):

$$
\begin{gathered}
(\forall \mathscr{U} \in \operatorname{Cov}(X))(\exists a \in A)\left(\forall a^{\prime}>a\right) p_{a^{\prime}}^{-1} \mathscr{U}_{a^{\prime}}<\mathscr{U}, \\
(\forall a \in A)\left(\exists a^{\prime}>a\right)\left(\forall a^{\prime \prime}>a^{\prime}\right) p_{a a^{\prime \prime}}\left(X_{a^{\prime \prime}}\right) \subset \operatorname{st}\left(p_{a}(X), \mathscr{U}_{a}\right) .
\end{gathered}
$$

The following is proved in [MW, Theorem 2.8].

(1.12) Theorem. An approximate map $\mathbf{p}=\left\{p_{a}: a \in A\right\}: X \rightarrow \mathscr{X}=\left\{X_{a}, \mathscr{U}_{a}\right.$, $\left.p_{a a^{\prime}}, A\right\}$ is an approximate resolution of the space $X$ if and only if it satisfies conditions (B1) and (B2).

(1.13) Remark. Let $\mathbb{X}=\left\{X_{a}, p_{a a^{\prime}}, A\right\}$ be an inverse system of compact spaces with an unbounded indexing set $A$ in the usual sense. Let $\mathbf{p}=\left\{p_{a}\right.$ : $a \in A\}: X \rightarrow \mathbb{X}$ be an inverse limit. By Remark (1.6), we may assume that $A$ is cofinite and that $\mathscr{X}=\left\{X_{a}, \mathscr{U}_{a}, p_{a a^{\prime}}, A\right\}$ is an approximate system associated with $\mathbb{X}$. Then $\mathbf{p}: X \rightarrow \mathscr{X}$ is an approximate resolution by (3.9) of [W1]. So any compact Hausdorff space has an approximate APOL-resolution. Moreover, any space has an approximate APOL-resolution by (3.15) of [M1]. Here, APOL denotes the collection of all approximate polyhedra (see [M1]).

For our discussion, we need the following basic lemmas about approximate resolutions of maps.

(1.14) Lemma. Let $\mathbf{q}: Y \rightarrow \mathscr{Y}$ be an approximate resolution over a cofinite index set $B$. Then for each positive integer $n$, there is an $n$-refinement function $s: B \rightarrow B$ of $\mathscr{Y}$ (see (7.4) of [MW]) with the property:

$$
\left(q_{b}, q_{b b^{\prime}} q_{b^{\prime}}\right)<\mathscr{V}_{b} \text { for any } b \text { and any } b^{\prime}>s(b) \text {. }
$$

By (A3) and (AS), for cofinite $B$, we can easily show Lemma (1.14).

(1.15) Lemma. Let $\mathbf{p}: X \rightarrow \mathscr{X}$ be an approximate resolution and let $\mathbf{q}: Y \rightarrow$ $\mathscr{Y}$ be an approximate APOL-resolution over a cofinite index set. Then every map $f: X \rightarrow Y$ admits a uniform approximate map $\mathbf{f}: \mathscr{X} \rightarrow \mathscr{Y}$ such that $(\mathbf{p}, \mathbf{q}, \mathbf{f})$ is an approximate resolution of $f($ see $(6.1)$ of $[\mathrm{MW}])$ and

$$
\left(f_{b} p_{f(b)}, q_{b} f\right)<\mathrm{st} \mathscr{V}_{b} \text { for } b \in B .
$$

Sometimes we say that $\mathbf{f}: \mathscr{X} \rightarrow \mathscr{Y}$ is an approximate resolution of $f$ with respect to $\mathbf{p}$ and $\mathbf{q}$ provided $(\mathbf{p}, \mathbf{q}, \mathbf{f})$ is an approximate resolution of $f$. More simply, when $\mathbf{p}$ and $\mathbf{q}$ are obvious in the paragraph, we say merely that $\mathbf{f}: \mathscr{X} \rightarrow \mathscr{Y}$ is an approximate resolution of $f$ provided that $(\mathbf{p}, \mathbf{q}, \mathbf{f})$ is an approximate resolution of $f$.

Proof. By (6.3) of [MW] there exists an approximate map $\mathbf{f}: \mathscr{X} \rightarrow \mathscr{Y}$ such that $(\mathbf{p}, \mathbf{q}, \mathbf{f})$ is an approximate resolution. By step (21) of the proof of $[\mathrm{MW}$, (6.3)] one may assume $(*)$. 
To modify $\mathbf{f}$ to a uniform $\mathbf{h}: \mathscr{X} \rightarrow \mathscr{Y}$, we choose an increasing function $\psi: B \rightarrow A$ such that $f<\psi$ and

(SF) $\left(p_{f(b) a^{\prime}} p_{a^{\prime} a^{\prime \prime}}, p_{f(b) a^{\prime \prime}}\right)<f_{b}^{-1} q_{b_{0} b}^{-1} \mathscr{V}_{b_{0}}$ for all $b_{0}<b$ and $\psi(b)<a^{\prime}<a^{\prime \prime}$,

$$
p_{f(b) a^{\prime}}^{-1} f_{b}^{-1} \mathscr{V}_{b}>\mathscr{U}_{a^{\prime}} \text { for any } a^{\prime}>\psi(b)
$$

$\left(\mathrm{RF}^{\prime}\right)$

$$
\left(p_{f(b)}, p_{f(b) a^{\prime}} p_{a^{\prime}}\right)<f_{b}^{-1} \mathscr{V}_{b} \text { for any } a^{\prime}>\psi(b)
$$

Such a shift function $\psi$ of $\mathbf{f}$ exists by (8.4) of [MW], (A3), and (AS). By Lemma (1.14), there exists a 2-refinement function $s: B \rightarrow B$ with (RF1). Let $h=\psi s: B \rightarrow A$ and $h_{b}=q_{b s(b)} f_{s(b)} p_{f s(b) \psi s(b)}: X_{h(b)} \rightarrow Y_{b}$ for $b \in B$. Then, using (SF) and (U), the shift $\mathbf{h}=\left\{h, h_{b}: b \in B\right\}: \mathscr{X} \rightarrow \mathscr{Y}$ of $\mathbf{f}$ by $\psi$ and $s$ is a uniform approximate map by $(8.5)$ and (8.6) (see especially the proof of (8.6)) of [MW].

We show that $\mathbf{h}$ is an approximate resolution of $f$. Take any $b \in B$. By $(\mathrm{RF} 1)^{\prime}$ and $(*)$,

$$
\begin{gathered}
\left(f_{s(b)} p_{f s(b)}, f_{s(b)} p_{f s(b) \psi s(b)} p_{\psi s(b)}\right)<\mathscr{V}_{s(b)}, \\
\left(q_{s(b)} f, f_{s(b)} p_{f s(b)}\right)<\mathrm{st} \mathscr{V}_{s(b)} .
\end{gathered}
$$

Since $s$ is a 2-refinement function, by (1) and (2)

$$
\left(q_{b s(b)} q_{s(b)} f, q_{b s(b)} f_{s(b)} p_{f s(b) \psi s(b)} p_{\psi s(b)}\right)<\mathscr{V}_{b} .
$$

By (RF1),

$$
\left(q_{b} f, q_{b s(b)} q_{s(b)} f\right)<\mathscr{V}_{b} .
$$

By (3) and (4), we have the condition $(*)$. By the same argument in the proof of $(5.5)$ of [MW], (*) implies (LAM) (see [MW]). Thus $\mathbf{h}$ is an approximate resolution of $f$. Hence $\mathbf{h}$ has the required properties.

(1.16) Lemma. Let $f: X \rightarrow Y$ and $g: Y \rightarrow Z$ be maps. Let $\mathbf{p}: X \rightarrow \mathscr{X}$, $\mathbf{q}: Y \rightarrow \mathscr{Y}$, and $\mathbf{r}: Z \rightarrow \mathscr{Z}$ be approximate resolutions. Let $\mathbf{f}: \mathscr{X} \rightarrow \mathscr{Y}$ and $\mathbf{g}: \mathscr{Y} \rightarrow \mathscr{Z}$ be uniform approximate maps. Let $(\mathbf{p}, \mathbf{q}, \mathbf{f})$ and $(\mathbf{q}, \mathbf{r}, \mathbf{g})$ be approximate resolutions of $f$ and $g$ with (*) in Lemma (1.15), respectively. If the index set of $\mathscr{Z}$ is cofinite, then there is a 2-refinement function $s$ for $\mathscr{Z}$ such that $s(\mathbf{g f}): \mathscr{X} \rightarrow \mathscr{Z}$ is a uniform approximate map and (p, $\mathbf{r}, s(\mathbf{g f}))$ is an approximate resolution of $g f$ with $(*)$ in Lemma (1.15).

Proof. By Lemma (1.14), there exists a 2-refinement function $s: C \rightarrow C$ for $\mathscr{Z}$ such that

$$
\left(r_{c}, r_{c c^{\prime}} r_{c^{\prime}}\right)<\mathscr{W}_{c} \text { for any } c^{\prime}>s(c) .
$$

Since $C$ is cofinite, we can assume that $s: C \rightarrow C^{*}$ (see (1.6) of [MW] for $C^{*}$ ) is increasing. By the proof of $(8.2)$ of [MW], $s(\mathbf{g f}): \mathscr{X} \rightarrow \mathscr{Z}$ is an approximate map. Since $\mathbf{f}, \mathbf{g}$ are uniform and $s$ is a 2-refinement function, it is easy to show that $s(\mathbf{g f})$ is uniform.

Since $\mathbf{f}$ and $\mathbf{g}$ have the property $(*)$, for any $c \in C$,

$$
\begin{gathered}
\left(r_{s(c)} g, g_{s(c)} q_{g s(c)}\right)<\mathrm{st} \mathscr{W}_{s(c)}, \\
\left(q_{g s(c)} f, f_{g s(c)} p_{f g s(c)}\right)<\mathrm{st} \mathscr{V}_{g s(c)} .
\end{gathered}
$$


Since $\mathbf{g}$ is uniform, (3) implies

$$
\left(g_{s(c)} q_{g s(c)} f, g_{s(c)} f_{g s(c)} p_{f g s(c)}\right)<\mathrm{st} \mathscr{W}_{s(c)} .
$$

By (2)

$$
\left(r_{s(c)} g f, g_{s(c)} q_{g s(c)} f\right)<\mathrm{st} \mathscr{W}_{s(c)} .
$$

Since $s$ is a 2-refinement function, by (4) and (5)

$$
\left(r_{c s(c)} r_{s(c)} g f, r_{c s(c)} g_{s(c)} f_{g s(c)} p_{f g s(c)}\right)<\mathscr{W}_{c} .
$$

$$
\left(r_{c} g f, r_{c s(c)} r_{s(c)} g f\right)<\mathscr{W}_{c} .
$$

By (6) and (7)

$$
\left(r_{c} g f, r_{c s(c)} g_{s(c)} f_{g s(c)} p_{f g s(c)}\right)<\mathrm{st} \mathscr{W}_{c} .
$$

Inequality $(8)$ means that $s($ gf $)$ satisfies $(*)$. Thus by the same argument in the proof of (5.5) of [MW], (8) implies that $s(\mathbf{g f})$ is an approximate resolution of $g f$.

Let $f: X \rightarrow Y$ and $g: Y \rightarrow Z$ be maps. Let $\mathbf{p}: X \rightarrow \mathscr{X}, \mathbf{q}: Y \rightarrow \mathscr{Y}$, and $\mathbf{r}: Z \rightarrow \mathscr{Z}$ be cofinite approximate APOL-resolutions.

(1.17) Corollary. If $\mathbf{f}: \mathscr{X} \rightarrow \mathscr{Y}, \mathbf{g}: \mathscr{Y} \rightarrow \mathscr{Z}$, and $\mathbf{h}: \mathscr{X} \rightarrow \mathscr{Z}$ are approximate resolutions of $f, g$, and $g f$, respectively, then $[\mathbf{g}][\mathbf{f}]=[\mathbf{h}]$.

Proof. By Lemma (1.15) there exist uniform approximate maps $\mathbf{f}_{1}: \mathscr{X} \rightarrow \mathscr{Y}$ and $\mathbf{g}_{1}: \mathscr{Y} \rightarrow \mathscr{Z}$ with $(*)$ such that $\left(\mathbf{p}, \mathbf{q}, \mathbf{f}_{1}\right)$ and $\left(\mathbf{q}, \mathbf{r}, \mathbf{g}_{1}\right)$ are approximate resolutions of $f$ and $g$, respectively. By Lemma (1.16), there exists a 2-refinement function $s$ for $\mathscr{Z}$ such that $s\left(\mathbf{g}_{1} \mathbf{f}_{1}\right): \mathscr{Z} \rightarrow \mathscr{Z}$ is a uniform approximate map and $\left(\mathbf{p}, \mathbf{r}, s\left(\mathbf{g}_{1} \mathbf{f}_{1}\right)\right)$ is a resolution of $g f$. Thus, by (7.6) of $[\mathrm{MW}],[\mathbf{f}]=\left[\mathbf{f}_{1}\right],[\mathbf{g}]=\left[\mathbf{g}_{1}\right]$, and $[\mathbf{h}]=\left[s\left(\mathbf{g}_{1} \mathbf{f}_{1}\right)\right]$. By the definition of composition, $\left[s\left(\mathbf{g}_{1} \mathbf{f}_{1}\right)\right]=\left[\mathbf{g}_{1}\right]\left[\mathbf{f}_{1}\right]$. Thus $[\mathbf{h}]=[\mathbf{g}][\mathbf{f}]$.

(1.18) Corollary. If $f: X \rightarrow Y$ is a homeomorphism, then [f] : $\mathscr{X} \rightarrow \mathscr{Y}$ is an isomorphism in the category APRO-APOL (see (8.16) of [MW]) for any approximate resolution $\mathbf{f}: \mathscr{X} \rightarrow \mathscr{Y}$ of $f$.

(1.19) Lemma. Let $\mathbf{p}=\left\{p_{a}: a \in A\right\}: X \rightarrow(\mathscr{X}, \mathscr{U})=\left\{X_{a}, \mathscr{U}_{a}, p_{a a^{\prime}}, A\right\}$ be an approximate resolution of a space $X$. Let $A=(A,<)$ be a cofinite, antisymmetric, infinite, directed set. Then for each $\mathscr{V}_{a} \in \operatorname{Cov}\left(X_{a}\right)$ with $\mathscr{V}_{a}<\mathscr{U}_{a}$, there exists an order $\ll$ in $A$ with the following conditions:

(i) $A^{*}=(A, \ll)$ is cofinite, antisymmetric, infinite and directed.

(ii) If $a \ll a^{\prime}$, then $a<a^{\prime}$.

(iii) If $a \ll a^{\prime}, a^{\prime}<a^{\prime \prime}$, and $a \neq a^{\prime}$, then $a \ll a^{\prime \prime}$.

There exists an approximate resolution $\mathbf{q}=\left\{q_{a}: a \in A^{*}\right\}: X \rightarrow(\mathscr{Y}, \mathscr{W})=$ $\left\{Y_{a}, \mathscr{W}_{a}, q_{a a^{\prime}}, A^{*}\right\}$ such that

(iv) each $Y_{a}, \mathscr{W}_{a}, q_{a a^{\prime}}$, and $q_{a}$ are $X_{a}, \mathscr{V}_{a}, p_{a a^{\prime}}$, and $p_{a}$, respectively,

(v) $\left(q_{a}, q_{a a^{\prime}} q_{a^{\prime}}\right)<\mathscr{V}_{a}$ for any $a \ll a^{\prime}$.

Moreover, if, in addition, $\mathscr{X}$ and $\mathbf{p}$ are commutative, we can achieve that $\mathscr{Y}$ and $\mathbf{q}$ are commutative.

Proof. Since p: $X \rightarrow(\mathscr{X}, \mathscr{U})$ is an approximate resolution, $(\mathscr{X}, \mathscr{U})$ satisfies the conditions (A1)-(A3) and p satisfies the conditions (AS), (R1), and (R2). 
Take any $\mathscr{V}_{a} \in \operatorname{Cov}\left(X_{a}\right)$. Since $A$ is infinite and directed, by (A2) for ( $\left.\mathscr{X}, \mathscr{U}\right)$ and (AS) for $\mathbf{p}$ there exists a function $\varphi: A \rightarrow A$ such that for each $a \in A$,

$$
\begin{gathered}
\varphi(a)>a \text { and } \varphi(a) \neq a, \\
\left(p_{a a_{1}} p_{a_{1} a_{2}}, p_{a a_{2}}\right)<\mathscr{V}_{a} \text { for any } a_{2}>a_{1}>\varphi(a), \\
\left(p_{a}, p_{a a_{1}} p_{a_{1}}\right)<\mathscr{V}_{a} \text { for any } a_{1}>\varphi(a) .
\end{gathered}
$$

We define a new order $\ll$ in the set $A$ as follows: Let $a, a^{\prime} \in A$. Then $a \ll a^{\prime}$ provided that (*) $a=a^{\prime}$ or $(* *) a \neq a^{\prime}$ and $\varphi(a)<a^{\prime}$. Since $(A,<)$ is cofinite, antisymmetric, infinite, and directed, it is easy, by (1), to show that $A^{*}=(A, \ll)$ satisfies the conditions (i)-(iii).

We define $(\mathscr{Y}, \mathscr{W})=\left\{Y_{a}, \mathscr{W}_{a}, q_{a a^{\prime}}, A^{*}\right\}$ as follows: For each $a \in A^{*}$, $Y_{a}=X_{a}$ and $\mathscr{W}_{a}=\mathscr{V}_{a} \in \operatorname{Cov}\left(Y_{a}\right)$, and for any $a \ll a^{\prime}, q_{a a^{\prime}}=p_{a a^{\prime}}: Y_{a^{\prime}}=$ $X_{a^{\prime}} \rightarrow Y_{b}=X_{a}$.

Claim 1. $(\mathscr{Y}, \mathscr{W})$ is an approximate inverse system.

To show Claim 1 we need to show the conditions (A1), (A2), and (A3) for

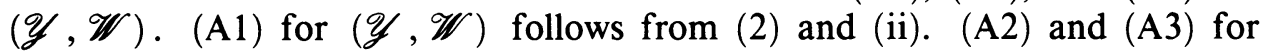
$(\mathscr{Y}, \mathscr{W})$ follows from $(\mathrm{A} 2)$ and $(\mathrm{A} 3)$ for $(\mathscr{X}, \mathscr{U})$, respectively.

Let $q_{a}=p_{a}: X \rightarrow Y_{a}=X_{a}$ for each $a \in A^{*}$. Then $\mathbf{q}=\left\{q_{a}: a \in A^{*}\right\}: X \rightarrow$ $(\mathscr{Y}, \mathscr{W})$ satisfies $(\mathrm{v})$ by (3). By definition, (iv) is satisfied.

Claim 2. $\mathbf{q}: X \rightarrow(\mathscr{Y}, \mathscr{W})$ is an approximate resolution.

To show Claim 2, we need to show conditions (AS), (R1), and (R2) for q. It is not difficult to show (AS), (R1), and (R2) for q by (AS), (R1), (R2) for p and (i)-(iii).

Hence by Claims 1 and 2, $\mathbf{q}$ is an approximate resolution. By the definitions of $(\mathscr{Y}, \mathscr{W})$ and $\mathbf{q}$, if, in addition $\mathscr{X}$ and $\mathbf{p}$ are commutative, then so are $\mathscr{Y}$ and $\mathbf{q}$. Hence this completes the proof of (1.19).

Let $[k]$ be the homotopy class of a map $k: X \rightarrow Y$, and let HTOP and HANR be the homotopy category of all spaces and of all ANR's, respectively.

(1.20) Lemma. Let $\mathbf{p}=\left\{p_{a}: a \in A\right\}: X \rightarrow(\mathscr{X}, \mathscr{U})=\left\{X_{a}, \mathscr{U}_{a}, p_{a a^{\prime}}, A\right\}$ be an approximate ANR resolution of a space $X$. We assume the following condition that for each $a \in A$,

(i) for any space $Y$ and maps $h, k: Y \rightarrow X_{a}$, if $(h, k)<s t^{4} \mathscr{U}_{a}$, then $h$ and $k$ are homotopic,

(ii) $\left(p_{a}, p_{a a^{\prime}} p_{a^{\prime}}\right)<\mathscr{U}_{a}$ for any $a^{\prime}>a$.

Then $H(\mathbf{p})=\left\{\left[p_{a}\right]: a \in A\right\}: X \rightarrow H(\mathscr{X})=\left\{X_{a},\left[p_{a a^{\prime}}\right], A\right\}$ is a ANR expansion (see p. 48 of [MS]).

Proof. By (A1) for $(\mathscr{X}, \mathscr{U})$ and (i), $\left[p_{a a^{\prime \prime}}\right]=\left[p_{a a^{\prime}}\right]\left[p_{a^{\prime} a^{\prime \prime}}\right]$ for $a<a^{\prime}<a^{\prime \prime}$. Thus $H(\mathscr{X})$ is an inverse system in HANR. Moreover, by (i) and (ii), $\left[p_{a}\right]=$ $\left[p_{a a^{\prime}}\right]\left[p_{a^{\prime}}\right]$ for $a<a^{\prime}$. Thus $h(\mathbf{p}): X \rightarrow H(\mathscr{X})$ is a system map. We need to show that

Claim 1. $H(\mathbf{p})$ satisfies the conditions (E1) and (E2) for ANR's (see p. 48 of [MS]).

By [M1] and Theorem 2 of p. 10 of [MS] we have an ANR-resolution $\mathbf{q}=$ $\left\{q_{b}: b \in B\right\}: X \rightarrow \mathscr{Y}=\left\{Y_{b}, q_{b b^{\prime}}, B\right\}$ of $X$ such that

$B$ is cofinite, antisymmetric, infinite, and directed, 
each $Y_{b}$ is an ANR.

By (3.7) of [W1], there exists an approximate resolution $\mathbf{r}=\left\{r_{c}: c \in C\right\}: X \rightarrow$ $(\mathscr{R}, \mathscr{V})=\left\{R_{c}, \mathscr{V}_{c}, r_{c c^{\prime}}, C\right\}$ such that

$C$ is cofinite, antisymmetric, infinite, and directed,

$$
(\mathscr{R}, \mathscr{V}) \text { and } \mathbf{r} \text { are commutative, }
$$
each $R_{c}$ is some $Y_{b}$.

By (4) and (2), each $R_{c}$ is an ANR. So there exists a $\mathscr{W}_{c} \in \operatorname{Cov}\left(R_{c}\right)$ such that for each $c \in C$,

$$
\mathscr{W}_{c}<\mathscr{V}_{c}
$$

for any space $Y$ and any maps $h, k: Y \rightarrow R_{c}$, if $(h, k)<\mathrm{st} \mathscr{W}_{c}$, then $h$ and $k$ are homotopic.

By Lemma (1.19), there exists an approximate resolution $\mathbf{s}=\left\{s_{d}: d \in D\right\}$ : $X \rightarrow(\mathscr{Z}, \mathscr{K})=\left\{Z_{d}, \mathscr{K}_{d}, s_{d d^{\prime}}, D\right\}$ such that

$$
D \text { is cofinite, antisymmetric, infinite, and directed, }
$$$$
(\mathscr{Z}, \mathscr{K}) \text { and } \mathbf{s} \text { are commutative, }
$$

Since $s$ is an approximate resolution, it satisfies the conditions (R1) and (R2) for ANR's. Then, by (9), s : $X \rightarrow \mathscr{Z}=\left\{Z_{d}, s_{d d^{\prime}}, D\right\}$ satisfies the conditions (R1) and (R2) for ANR's in the sense of [MS]. Thus, by (2), (5), and (9), s : $X \rightarrow \mathscr{Z}$ is an ANR-resolution. Hence by Theorem 2 of p. 75 of [MS], $H(\mathbf{s})=\left\{\left[s_{d}\right]: d \in D\right\}: X \rightarrow H(\mathscr{Z})=\left\{Z_{d},\left[s_{d d^{\prime}}\right], D\right\}$ is an HANR-expansion, i.e.,

(11) $\quad H(\mathbf{s}): X \rightarrow H(\mathscr{Z})$ satisfies the conditions (E1) and (E2) for ANR's.

Since $\mathbf{p}: X \rightarrow(\mathscr{X}, \mathscr{U})$ and $\mathbf{s}: X \rightarrow(\mathscr{Z}, \mathscr{K})$ are approximate resolutions with cofinite index sets, by Lemma $(1.15)$ we have uniform approximate maps $\mathbf{f}=\left\{f, f_{d}: d \in D\right\}:(\mathscr{X}, \mathscr{U}) \rightarrow(\mathscr{Z}, \mathscr{K})$ and $\mathbf{g}=\left\{g, g_{a}: a \in A\right\}:(\mathscr{Z}, \mathscr{K}) \rightarrow$ $(\mathscr{X}, \mathscr{U})$ such that $(\mathbf{p}, \mathbf{s}, \mathbf{f})$ and $(\mathbf{s}, \mathbf{p}, \mathbf{g})$ are approximate resolutions of $1_{X}$, i.e.,

$$
\begin{gathered}
\left(f_{d} p_{f(d)}, s_{d} 1_{X}\right)<\mathrm{st} \mathscr{K}_{d} \text { for } d \in D, \\
\left(g_{a} s_{g(a)}, p_{a} 1_{X}\right)<\mathrm{st} \mathscr{U}_{a} \text { for } a \in A, \\
g_{a}^{-1} \mathscr{U}_{a}>\mathscr{K}_{g(a)} \quad \text { for } a \in A .
\end{gathered}
$$

Since $\mathbf{f}$ is an approximate map, for each $d<d^{\prime}$ there exists an $a \in A$ with $a>f(d), f\left(d^{\prime}\right)$ such that

$$
\left(f_{d} p_{f(d) a}, s_{d d^{\prime}} f_{d^{\prime}} p_{f\left(d^{\prime}\right) a}\right)<\mathrm{st} \mathscr{K}_{d} .
$$

By (10), $Z_{d}=Y_{c}$ and $\mathscr{K}_{d}=\mathscr{W}_{c}$ for some $c \in C$. By (7) and (15)

$$
\left[f_{d}\right]\left[p_{f(d) a}\right]=\left[s_{d d^{\prime}}\right]\left[f_{d^{\prime}}\right]\left[p_{f\left(d^{\prime}\right) a}\right] \text {. }
$$


Equality (16) means that $H(\mathbf{f})=\left\{f,\left[f_{d}\right]: d \in D\right\}: H(\mathscr{X}) \rightarrow H(\mathscr{Z})$ is a morphism of inverse systems in HANR. Similarly, $H(\mathbf{g}): H(\mathscr{Z}) \rightarrow H(\mathscr{X})$ is also.

Claim 2. $H(\mathbf{g}) H(\mathbf{f}) \sim 1_{H(\mathscr{L})}$ and $H(\mathbf{f}) H(\mathbf{g}) \sim 1_{H(\mathscr{E})}$.

By (7), (12), and (13),

$$
\begin{aligned}
& {\left[f_{d}\right]\left[p_{f(d)}\right]=\left[s_{d}\right] \quad \text { for } d \in D,} \\
& {\left[g_{a}\right]\left[s_{g(a)}\right]=\left[p_{a}\right] \quad \text { for } a \in A .}
\end{aligned}
$$

For each $d \in D$, there exists a $d^{\prime} \in D$ such that $d^{\prime}>d, g f(d)$. By (17) and (18), $\left[s_{d d^{\prime}}\right]\left[s_{d^{\prime}}\right]=\left[s_{d}\right]=\left[f_{d}\right]\left[p_{f(d)}\right]=\left[f_{d}\right]\left[g_{f(d)}\right]\left[s_{g f(d)}\right]=\left[f_{d}\right]\left[g_{f(d)}\right]\left[s_{g f(d) d^{\prime}}\right]\left[s_{d^{\prime}}\right]$. Since $H(\mathbf{s})$ satisfies (E2) by (11), there exists a $d^{\prime \prime}>d^{\prime}$ such that

$$
\left[s_{d d^{\prime \prime}}\right]=\left[f_{d}\right]\left[g_{f(d)}\right]\left[s_{g f(d) d^{\prime}}\right]\left[s_{d^{\prime} d^{\prime \prime}}\right] .
$$

This means that $H(\mathbf{f}) H(\mathbf{g}) \sim 1_{H(\mathscr{Z})}$.

Now, take any $a \in A$. By (12) and (14), $\left(f_{g(a)} p_{f g(a)}, s_{g(a)}\right)<$ st $\mathscr{K}_{g(a)}<$ $g_{a}^{-1}\left(\mathbf{s t} \mathscr{U}_{a}\right)$. Thus

$$
\left(g_{a} f_{g(a)} p_{f g(a)}, g_{a} s_{g(a)}\right)<\mathrm{st} \mathscr{U}_{a} .
$$

Since $\left(g_{a} f_{g(a)}\right)^{-1} \mathscr{U}_{a} \in \operatorname{Cov}\left(X_{f g(a)}\right)$, by (AS) for p there exists an $a_{1}>f g(a)$ such that $\left(p_{f g(a)}, p_{f g(a) a^{\prime}} p_{a^{\prime}}\right)<\left(g_{a} f_{g(a)}\right)^{-1} \mathscr{U}_{a}$ for any $a^{\prime}>a_{1}$. Then

$$
\left(g_{a} f_{g(a)} p_{f g(a)}, g_{a} f_{g(a)} p_{f g(a) a^{\prime}} p_{a^{\prime}}\right)<\mathscr{U}_{a} \text { for any } a^{\prime}>a_{1} \text {. }
$$

By (AS) for $\mathbf{p}$, there exists an $a_{2}>a, a_{1}$ such that

$$
\left(p_{a}, p_{a a^{\prime}} p_{a^{\prime}}\right)<\mathscr{U}_{a} \text { for } a^{\prime}>a_{2} .
$$

By (21) for $a^{\prime}=a_{2}$ and (20), (13), and (22) for $a^{\prime}=a_{2}$,

$$
\left(g_{a} f_{g(a)} p_{f g(a) a_{2}} p_{a_{2}}, p_{a a_{2}} p_{a_{2}}\right)<\mathrm{st}^{3} \mathscr{U}_{a} .
$$

Since $\mathbf{p}: X \rightarrow(\mathscr{X}, \mathscr{U})$ is an approximate ANR-resolution, by (1.12) it satisfies the conditions (B1) and (B2). By (2.14) of [MW], it satisfies (R3) for ANR's. By (R3) for $\mathbf{p}$ and (23) there exists an $a_{3}>a_{2}$ such that

$$
\left(g_{a} f_{g(a)} p_{f g(a) a_{2}} p_{a_{2}} p_{a_{3}}, p_{a a_{2}} p_{a_{2} a_{3}}\right)<\mathrm{st}^{4} \mathscr{U}_{a} .
$$

By (i) and (24)

$$
\left[g_{a}\right]\left[f_{g(a)}\right]\left[p_{f g(a) a_{3}}\right]=\left[p_{a a_{3}}\right] .
$$

This means that $H(\mathbf{g}) H(\mathbf{f}) \sim 1_{H(\mathscr{L})}$. Hence we have Claim 2.

Now, we show Claim 1. First, we consider the condition (E1). Take any map $h: X \rightarrow P$ into an ANR. Since $H(\mathbf{s})$ satisfies (E1) by (11), there exist a $d \in D$ and a map $k: Z_{d} \rightarrow P$ such that

$$
[h]=\left[k s_{d}\right] .
$$

By (17) and (26), $[h]=\left[k f_{d}\right]\left[p_{f(d)}\right]$. This means that $H(\mathbf{p})$ satisfies (E1) for ANR's.

Next, we consider the condition (E2) for ANR's. Take any $a \in A$ and any maps $h, k: X_{a} \rightarrow P$ into an ANR $P$ such that

$$
\left[h p_{a}\right]=\left[k p_{a}\right] \text {. }
$$


By (18) and (27),

$$
\left[h g_{a} s_{g(a)}\right]=\left[k g_{a} s_{g(a)}\right] \text {. }
$$

Since $H(\mathbf{s})$ satisfies (E2) for ANR's by (11), by (28) there exists a $d_{1}>g(a)$ such that

$$
\left[h g_{a} s_{g(a) d_{1}}\right]=\left[k g_{a} s_{g(a) d_{1}}\right] .
$$

By (16) for $d_{1}>g(a)$, there exists an $a_{0}>a_{3}, f\left(d_{1}\right)$ such that

$$
\left[f_{g(a)} p_{f g(a) a_{0}}\right]=\left[s_{g(a) d_{1}} f_{d_{1}} p_{f\left(d_{1}\right) a_{0}}\right]
$$

Since

$$
\begin{aligned}
{\left[p_{a a_{0}}\right] } & =\left[p_{a a_{3}}\right]\left[p_{a_{3} a_{0}}\right]=\left[g_{a} f_{g(a)} p_{f g(a) a_{3}}\right]\left[p_{a_{3} a_{0}}\right] \\
& =\left[g_{a}\right]\left[f_{g(a)} p_{f g(a) a_{0}}\right]=\left[g_{a}\right]\left[s_{g(a) d_{1}} f_{d_{1}} p_{f\left(d_{1}\right) a_{0}}\right]
\end{aligned}
$$

by $(25)$ and (30),

$$
\left[p_{a a_{0}}\right]=\left[g_{a} s_{g(a) d_{1}} f_{d_{1}} p_{f\left(d_{1}\right) a_{0}}\right] .
$$

By (29) and (31), $\left[h p_{a a_{0}}\right]=\left[h g_{a} s_{g(a) d_{1}}\right]\left[f_{d_{1}} p_{f\left(d_{1}\right) a_{0}}\right]=\left[k g_{a} s_{g(a) d_{1}}\right]\left[f_{d_{1}} p_{f\left(d_{1}\right) a_{0}}\right]=$ $\left[k p_{a a_{0}}\right]$, i.e.,

$$
\left[h p_{a a_{0}}\right]=\left[k p_{a a_{0}}\right] .
$$

(32) means that $H(\mathbf{s})$ satisfies (E2). Thus we have Claim 1 and hence we have completed the proof.

\section{APPROXIMATE NEARNESS OF SYSTEM MAPS}

Let $(\mathscr{X}, \mathscr{U})=\left\{X_{a}, \mathscr{U}_{a}, p_{a a^{\prime}}, A\right\}$ and $(\mathscr{Y}, \mathscr{V})=\left\{Y_{b}, \mathscr{V}_{b}, q_{b b^{\prime}}, B\right\}$ be approximate inverse systems with cofinite directed sets. Let $\mathbf{f}=\left\{f, f_{b} ; b \in B\right\}$, $\mathbf{g}=\left\{g, g_{b}: b \in B\right\}:(\mathscr{X}, \mathscr{U}) \rightarrow(\mathscr{Y}, \mathscr{V})$ be approximate maps. We consider approximate nearness as follows:

(AN) For each $b \in B$, there exist a $b_{0}>b$ and an $a_{0}>f\left(b_{0}\right), g\left(b_{0}\right)$ with the property: for each $a>a_{0}$ there exist an $x \in X_{a}$ and a $V \in \mathscr{V}_{b}$ such that $q_{b b_{0}} f_{b_{0}} p_{f\left(b_{0}\right) a}(x), q_{b b_{0}} g_{b_{0}} p_{g\left(b_{0}\right) a}(x) \in V$.

(2.1) Lemma. Let $\mathbf{f}^{\prime}=\left\{f^{\prime}, f_{b}^{\prime}: b \in B\right\}, \mathbf{g}^{\prime}=\left\{g^{\prime}, g_{b}^{\prime}: b \in B\right\}:(\mathscr{X}, \mathscr{U}) \rightarrow$ $(\mathscr{Y}, \mathscr{V})$ be approximate maps. Let $\mathbf{f} \sim \mathbf{f}^{\prime}$ and $\mathbf{g} \sim \mathbf{g}^{\prime}$. If $\mathbf{f}$ and $\mathbf{g}$ satisfy condition (AN), then so do $\mathbf{f}^{\prime}$ and $\mathbf{g}^{\prime}$.

Proof. Since $\mathbf{f} \sim \mathbf{f}^{\prime}$ and $\mathbf{g} \sim \mathbf{g}^{\prime}$, by (7.8) of [MW] there exist positive integers $n, m$ with the following properties: For each $b \in B$ there exists $a>$ $f(b), f^{\prime}(b), g(b), g^{\prime}(b)$ such that

$$
\begin{aligned}
& \left(f_{b} p_{f(b) a^{\prime}}, \quad f_{b}^{\prime} p_{f^{\prime}(b) a^{\prime}}\right)<\mathbf{s t}^{n} \mathscr{V}_{b}, \\
& \left(g_{b} p_{g(b) a^{\prime}}, \quad g_{b}^{\prime} p_{g^{\prime}(b) a^{\prime}}\right)<\mathbf{s t}^{m} \mathscr{V}_{b}
\end{aligned}
$$

for each $a^{\prime}>a$.

Take any $b \in B$. By (A3) there exists a $b_{1}>b$ such that

$$
q_{b b^{\prime}}^{-1} \mathscr{V}_{b}>\mathrm{st}^{n+m+2} \mathscr{V}_{b^{\prime}} \text { for any } b^{\prime}>b_{1} \text {. }
$$

There exist a $b_{2}>b_{1}$ and an $a_{1}>f\left(b_{2}\right), g\left(b_{2}\right)$ satisfying condition (AN) for $\mathbf{f}, \mathbf{g}$, and $b_{1}$. 
By (AM), there exists an $a_{2}>f\left(b_{1}\right), f\left(b_{2}\right), a_{1}, g\left(b_{1}\right), g\left(b_{2}\right)$ such that

$$
\begin{gathered}
\left(q_{b_{1} b_{2}} f_{b_{2}} p_{f\left(b_{2}\right) a^{\prime}}, f_{b_{1}} p_{f\left(b_{1}\right) a^{\prime}}\right)<\mathrm{st} \mathscr{V}_{b_{1}}, \\
\left(q_{b_{1} b_{2}} g_{b_{2}} p_{g\left(b_{2}\right) a^{\prime}}, g_{b_{1}} p_{g\left(b_{1}\right) a^{\prime}}\right)<\mathrm{st} \mathscr{V}_{b_{1}}
\end{gathered}
$$

for any $a^{\prime}>a_{2}$.

By (1) and (2), there exists an $a_{3}>a_{2}, f^{\prime}\left(b_{1}\right), g^{\prime}\left(b_{1}\right)$ such that

$$
\begin{aligned}
& \left(f_{b_{1}} p_{f\left(b_{1}\right) a^{\prime}}, f_{b_{1}}^{\prime} p_{f^{\prime}\left(b_{1}\right) a^{\prime}}\right)<\mathbf{s t}^{n} \mathscr{V}_{b_{1}}, \\
& \left(g_{b_{1}} p_{g\left(b_{1}\right) a^{\prime}}, g_{b_{1}}^{\prime} p_{g^{\prime}\left(b_{1}\right) a^{\prime}}\right)<\mathbf{s t}^{m} \mathscr{V}_{b_{1}}
\end{aligned}
$$

for any $a^{\prime}>a_{3}$.

Take any $a^{\prime}>a_{3}$. By the choice of $a_{1}$ and $b_{2}$, there exist an $x \in X_{a^{\prime}}$ and a $V_{0} \in \mathscr{V}_{b_{1}}$ such that

$$
q_{b_{1} b_{2}} f_{b_{2}} p_{f\left(b_{2}\right) a^{\prime}}(x), q_{b_{1} b_{2}} g_{b_{2}} p_{g\left(b_{2}\right) a^{\prime}}(x) \in V_{0} .
$$

By (4) and (5), there exist $V_{1}, V_{2} \in \mathrm{st} \mathscr{V}_{b_{1}}$ such that

$$
\begin{aligned}
& q_{b_{1} b_{2}} f_{b_{2}} p_{f\left(b_{2}\right) a^{\prime}}(x), f_{b_{1}} p_{f\left(b_{1}\right) a^{\prime}}(x) \in V_{1}, \\
& q_{b_{1} b_{2}} g_{b_{2}} p_{g\left(b_{2}\right) a^{\prime}}(x), \quad g_{b_{1}} p_{g\left(b_{1}\right) a^{\prime}}(x) \in V_{2} .
\end{aligned}
$$

By (6) and (7), there exist a $V_{3} \in \mathbf{s t}^{n} \mathscr{V}_{b_{1}}$ and a $V_{4} \in \mathbf{s t}^{m} \mathscr{V}_{b_{1}}$ such that

$$
\begin{aligned}
& f_{b_{1}} p_{f\left(b_{1}\right) a^{\prime}}(x), f_{b_{1}}^{\prime} p_{f^{\prime}\left(b_{1}\right) a^{\prime}}(x) \in V_{3}, \\
& g_{b_{1}} p_{g\left(b_{1}\right) a^{\prime}}(x), \quad g_{b_{1}}^{\prime} p_{g^{\prime}\left(b_{1}\right) a^{\prime}}(x) \in V_{4} .
\end{aligned}
$$

By (11), (9), (8), (10), and (12), there exists a $V_{5} \in \mathbf{s t}^{n+m+2} \mathscr{V}_{b_{1}}$ such that

$$
f_{b_{1}}^{\prime} p_{f^{\prime}\left(b_{1}\right) a^{\prime}}(x), \quad g_{b_{1}}^{\prime} p_{g^{\prime}\left(b_{1}\right) a^{\prime}}(x) \in V_{3} \cup V_{1} \cup V_{0} \cup V_{2} \cup V_{4} \subset V_{5} .
$$

By (3), there exists a $V_{6} \in \mathscr{V}_{b}$ such that $q_{b b_{1}}^{-1} V_{6} \supset V_{5}$. Then, by (13), we have that

$$
q_{b b_{1}} f_{b_{1}}^{\prime} p_{f^{\prime}\left(b_{1}\right) a^{\prime}}(x), q_{b b_{1}} g_{b_{1}}^{\prime} p_{g^{\prime}\left(b_{1}\right) a^{\prime}}(x) \in V_{6} .
$$

(14) means that $\mathbf{f}^{\prime}$ and $\mathbf{g}^{\prime}$ satisfy property (AN).

(2.2) Lemma. Let $\mathbf{f}, \mathbf{g}:(\mathscr{X}, \mathscr{U}) \rightarrow(\mathscr{Y}, \mathscr{V})$ be approximate maps. $\mathbf{f}$ and $\mathbf{g}$ satisfy property (AN) if and only if they satisfy the following condition:

(AN1) There exists an integer $k \geq 0$ having the property: for any $b \in B$ there exist an $a>f(b), g(b)$ such that for any $a^{\prime}>a$ there exist an $x \in X_{a^{\prime}}$ and $a V \in \mathrm{st}^{k} \mathscr{V}_{b}$ with $f_{b} p_{f(b) a^{\prime}}(x), g_{b} p_{g(b) a^{\prime}}(x) \in V$.

Proof. (AN) $\Rightarrow$ (AN1). Take any $b \in B$. By (AN), there exist a $b_{0}>b$ and an $a_{0}>f\left(b_{0}\right), g\left(b_{0}\right)$ satisfying property (AN). By (AM) of (1.10) there exists an $a_{1}>a_{0}, f(b), g(b)$ such that

$$
\begin{gathered}
\left(q_{b b_{0}} f_{b_{0}} p_{f\left(b_{0}\right) a^{\prime}}, f_{b} p_{f(b) a^{\prime}}\right)<\mathrm{st} \mathscr{V}_{b}, \\
\left(q_{b b_{0}} g_{b_{0}} p_{g\left(b_{0}\right) a^{\prime}}, g_{b} p_{g(b) a^{\prime}}\right)<\mathrm{st} \mathscr{\mathscr { V }}_{b}
\end{gathered}
$$

for any $a^{\prime}>a_{1}$. 
Take any $a^{\prime}>a_{1}$. By the choices of $a_{0}$ and $b_{0}$, there exist an $x \in X_{a^{\prime}}$ and a $V_{0} \in \mathscr{V}_{b}$ such that

$$
q_{b b_{0}} f_{b_{0}} p_{f\left(b_{0}\right) a^{\prime}}(x), q_{b b_{0}} g_{b_{0}} p_{g\left(b_{0}\right) a^{\prime}}(x) \in V_{0}
$$

By (1) and (2), there exist $V_{1}, V_{2} \in \mathrm{st} \mathscr{V}_{b}$ such that

$$
\begin{aligned}
& q_{b b_{0}} f_{b_{0}} p_{f\left(b_{0}\right) a^{\prime}}(x), \quad f_{b} p_{f(b) a^{\prime}}(x) \in V_{1}, \\
& q_{b b_{0}} g_{b_{0}} p_{g\left(b_{0}\right) a^{\prime}}(x), \quad g_{b} p_{g(b) a^{\prime}}(x) \in V_{2} .
\end{aligned}
$$

By (4), (3), and (5), there exists a $V_{3} \in \mathrm{st}^{2} \mathscr{V}_{b}$ such that $f_{b} p_{f(b) a^{\prime}}(x), g_{b} p_{g(b) a^{\prime}}(x)$ $\in V_{1} \cup V_{0} \cup V_{2} \subset V_{3}$. Therefore, $\mathbf{f}$ and $\mathbf{g}$ satisfy property (AN1) for $k=2$.

$(\mathrm{AN} 1) \Rightarrow(\mathrm{AN})$. By assumption, there exists an integer $k \geq 0$ having the property (AN1). Take any $b \in B$. By (A3) there exist a $b_{0}>b$ such that

$$
q_{b b^{\prime}}^{-1} \mathscr{V}_{b}>\mathrm{st}^{k} \mathscr{V}_{b^{\prime}} \text { for any } b^{\prime}>b_{0}
$$

By the choice of $k$, there exists an $a_{0}>f\left(b_{0}\right), g\left(b_{0}\right)$ with the property: for any $a^{\prime}>a_{0}$ there exist an $x \in X_{a^{\prime}}$ and a $V_{0} \in \mathrm{st}^{k} \mathscr{V}_{b_{0}}$ such that

$$
f_{b_{0}} p_{f\left(b_{0}\right) a^{\prime}}(x), g_{b_{0}} p_{g\left(b_{0}\right) a^{\prime}}(x) \in V_{0} \text {. }
$$

By (6) there exists a $V_{1} \in \mathscr{V}_{b}$ such that $q_{b b_{0}}^{-1} V_{1} \supset V_{0}$. Thus, by (7),

$$
q_{b b_{0}} f_{b_{0}} p_{f\left(b_{0}\right) a^{\prime}}(x), q_{b b_{0}} g_{b_{0}} p_{g\left(b_{0}\right) a^{\prime}}(x) \in V_{1} \text {. }
$$

This means that $\mathbf{f}$ and $\mathbf{g}$ satisfy $(\mathrm{AN})$.

(2.3) Lemma. Condition (AN) is equivalent to the following

(AN2) For each $b \in B$ and each $\mathscr{V} \in \operatorname{Cov}\left(Y_{b}\right)$, there exist $a b_{0}>b$ and an $a_{0}>f\left(b_{0}\right), g\left(b_{0}\right)$ with the property: for each $a^{\prime}>a_{0}$ there exist an $x \in X_{a^{\prime}}$ and $a V \in \mathscr{V}$ such that $q_{b b_{0}} f_{b_{0}} p_{f\left(b_{0}\right) a^{\prime}}(x), q_{b b_{0}} g_{b_{0}} p_{g\left(b_{0}\right) a^{\prime}}(x) \in V$.

Proof. Clearly (AN2) implies (AN). We show that (AN) implies (AN2). Take any $b \in B$ and any $\mathscr{V} \in \operatorname{Cov}\left(Y_{b}\right)$. Take a $\mathscr{V}^{\prime} \in \operatorname{Cov}\left(Y_{b}\right)$ such that

$$
\text { st } \mathscr{V}^{\prime}<\mathscr{V} \text {. }
$$

By (A2) there exists a $b_{1}>b$ such that

$$
\left(q_{b b^{\prime}} q_{b^{\prime} b^{\prime \prime}}, q_{b b^{\prime \prime}}\right)<\mathscr{V}^{\prime} \text { for any } b^{\prime \prime}>b^{\prime}>b_{1} \text {. }
$$

By (A3) there exists a $b_{2}>b_{1}$ such that

$$
q_{b b^{\prime}}^{-1} \mathscr{V}^{\prime}>\mathscr{V}_{b^{\prime}} \text { for any } b^{\prime}>b_{2}
$$

By (AN) there exist a $b_{3}>b_{2}$ and an $a_{0}>f\left(b_{3}\right), g\left(b_{3}\right)$ with the property: for any $a^{\prime}>a_{0}$ there exist an $x \in X_{a^{\prime}}$ and a $V_{0} \in \mathscr{V}_{b_{2}}$ such that

$$
q_{b_{2} b_{3}} f_{b_{3}} p_{f\left(b_{3}\right) a^{\prime}}(x), q_{b_{2} b_{3}} g_{b_{3}} p_{g\left(b_{3}\right) a^{\prime}}(x) \in V_{0} \text {. }
$$

By (3), there exists a $V_{1} \in \mathscr{V}^{\prime}$ such that $q_{b b_{2}}^{-1} V_{1} \supset V_{0}$. Thus, by (4), we have that

$$
q_{b b_{2}} q_{b_{2} b_{3}} f_{b_{3}} p_{f\left(b_{3}\right) a^{\prime}}(x), q_{b b_{2}} q_{b_{2} b_{3}} g_{b_{3}} p_{g\left(b_{3}\right) a^{\prime}}(x) \in V_{1} .
$$

By (2) there exist $V_{2}, V_{3} \in \mathscr{V}^{\prime}$ such that

$$
q_{b b_{2}} q_{b_{2} b_{3}} f_{b_{3}} p_{f\left(b_{3}\right) a^{\prime}}(x), q_{b b_{3}} f_{b_{3}} p_{f\left(b_{3}\right) a^{\prime}}(x) \in V_{2},
$$




$$
q_{b b_{2}} q_{b_{2} b_{3}} g_{b_{3}} p_{g\left(b_{3}\right) a^{\prime}}(x), q_{b b_{3}} g_{b_{3}} p_{g\left(b_{3}\right) a^{\prime}}(x) \in V_{3} .
$$

By (6), (5), (7), and (1), there exists a $V \in \mathscr{V}$ such that

$$
q_{b b_{3}} f_{b_{3}} p_{f\left(b_{3}\right) a^{\prime}}(x), q_{b b_{3}} g_{b_{3}} p_{g\left(b_{3}\right) a^{\prime}}(x) \in V_{2} \cup V_{1} \cup V_{3} \subset \operatorname{st}\left(V_{1}, \mathscr{V}^{\prime}\right) \subset V .
$$

This means that $\mathbf{f}$ and $\mathbf{g}$ satisfy property (AN2).

(2.4) Lemma. Let $\mathbf{f}, \mathbf{g}:(\mathscr{X}, \mathscr{U}) \rightarrow(\mathscr{Y}, \mathscr{V})$ and $\mathbf{h}=\left\{h, h_{c}: c \in C\right\}:$ $(\mathscr{Y}, \mathscr{V}) \rightarrow(\mathscr{Z}, \mathscr{W})=\left\{\mathscr{Z}_{c}, \mathscr{W}_{c}, r_{c c^{\prime}}, C\right\}$ be approximate maps. Let $\mathbf{h}$ be uniform. If $\mathbf{f}$ and $\mathbf{g}$ satisfy property (AN1), then $s(\mathbf{h f}), s(\mathbf{h g}):(\mathscr{X}, \mathscr{U}) \rightarrow$ $(\mathscr{Y}, \mathscr{V})$ satisfy $(\mathrm{AN})$ for some 2-refinement function s of $(\mathscr{Z}, \mathscr{W})$.

Proof. Since $\mathbf{f}$ and $\mathbf{g}$ satisfy property (AN1), there exists an integer $k \geq 0$ having the property (AN1). Let $s: C \rightarrow C$ be a $(k+2)$-refinement function for $(\mathscr{Z}, \mathscr{W})$. Take any $c \in C$. There exists an $a_{0}>f h s(c), g h s(c)$ such that for any $a^{\prime}>a_{0}$ there exist an $x \in X_{a^{\prime}}$ and a $V \in \mathrm{st}^{k} \mathscr{V}_{h s(c)}$ with

$$
f_{h s(c)} p_{f h s(c) a^{\prime}}(x), g_{h s(c)} p_{g h s(c) a^{\prime}}(x) \in V .
$$

Since $\mathbf{h}$ is uniform, $h_{s(c)}^{-1}\left(\mathscr{W}_{s(c)}\right)>\mathscr{V}_{h s(c)}$. Thus

$$
h_{s(c)}^{-1}\left(\mathbf{s t}^{k} \mathscr{W}_{s(c)}\right)>\mathbf{s t}^{k} \mathscr{V}_{h(c)} \text {. }
$$

Since $s$ is a $(k+2)$-refinement,

$$
r_{c, s(c)}^{-1}\left(\mathscr{W}_{c}\right)>\mathrm{st}^{k+2} \mathscr{W}_{s(c)}>\mathrm{st}^{k} \mathscr{W}_{s(c)} .
$$

By (1), (2), and (3), there exists a $W \in \mathscr{W}_{c}$ such that

$$
r_{c s(c)} h_{s(c)} f_{h s(c)} p_{f h s(c) a^{\prime}}(x), r_{c s(c)} h_{s(c)} g_{h s(c)} p_{g h s(c) a^{\prime}}(x) \in W .
$$

This means that $s(\mathbf{h f})$ and $s(\mathbf{h g})$ satisfy condition (AN).

Now, by Lemma (2.1), we can say that [f] and [g] satisfy property (AN) provided any/some representatives $\mathbf{f}$ and $\mathbf{g}$ satisfy property (AN). By Lemmas (2.1), (2.2), and (2.4), we can easily show that

(2.5) Corollary. Let $\mathbf{f}, \mathbf{g}:(\mathscr{X}, \mathscr{U}) \rightarrow(\mathscr{Y}, \mathscr{V})$ and $\mathbf{h}:(\mathscr{Y}, \mathscr{V}) \rightarrow(\mathscr{Z}, \mathscr{W})$ be approximate maps. If $[\mathbf{f}]$ and $[\mathbf{g}]$ satisfy property (AN), then so do $[\mathbf{h}][\mathbf{f}]$ and $[\mathbf{h}][\mathbf{g}]$.

(2.6) Lemma. Let $\mathbf{k}=\left\{k, k_{a}: a \in A\right\}:(\mathscr{Z}, \mathscr{W}) \rightarrow(\mathscr{X}, \mathscr{U})$ and $\mathbf{f}, \mathbf{g}:$ $(\mathscr{X}, \mathscr{U}) \rightarrow(\mathscr{Y}, \mathscr{V})$ be approximate system maps. Let $\mathbf{f}$ and $\mathbf{g}$ be uniform. Let $s: B \rightarrow B$ be a 2-refinement function for $(\mathscr{Y}, \mathscr{V})$. If $s(\mathbf{f k})$ and $s(\mathbf{g k})$ satisfy property (AN), then so do $\mathbf{f}$ and $\mathbf{g}$.

Proof. Take any $b \in B$ and a $\mathscr{V} \in \operatorname{Cov}\left(Y_{b}\right)$ such that

$$
\mathbf{s t}^{2} \mathscr{V}<\mathscr{V}_{b} \text {. }
$$

By (A2) there exists a $b_{1}>b$ such that

$$
\left(q_{b b^{\prime}} q_{b^{\prime} b^{\prime \prime}}, q_{b b^{\prime \prime}}\right)<\mathscr{V}
$$

for any $b^{\prime \prime}>b^{\prime}>b_{1}$. By (2.2) there exists an integer $n \geq 0$ having the property in (AN1) for $s(\mathbf{f k})$ and $s(\mathbf{g k})$. By (A3), there exists a $b_{2}>b_{1}$ such that

$$
q_{b b^{\prime}}^{-1} \mathscr{V}>\mathrm{st}^{n+2} \mathscr{V}_{b^{\prime}}
$$


for any $b^{\prime}>b_{2}$. Take an $a_{1}>f s\left(b_{2}\right), g s\left(b_{2}\right)$ and any $a^{\prime}>a_{1}$. By (AM) there exists a $c_{1}>k f s\left(b_{2}\right), k\left(a^{\prime}\right), k g s\left(b_{2}\right)$ such that

$$
\begin{gathered}
\left(p_{f s\left(b_{2}\right) a^{\prime}} k_{a^{\prime}} r_{k\left(a^{\prime}\right) c^{\prime}}, k_{f s\left(b_{2}\right)} r_{k f s\left(b_{2}\right) c^{\prime}}\right)<s t \mathscr{U}_{f s\left(b_{2}\right)}, \\
\left(p_{g s\left(b_{2}\right) a^{\prime}} k_{a^{\prime}} r_{k\left(a^{\prime}\right) c^{\prime}}, k_{g s\left(b_{2}\right)} r_{k g s\left(b_{2}\right) c^{\prime}}\right)<\mathrm{st} \mathscr{U}_{g s\left(b_{2}\right)}
\end{gathered}
$$

for any $c^{\prime}>c_{1}$.

Since $\mathbf{f}$ and $\mathbf{g}$ are uniform, we have $f_{s\left(b_{2}\right)}^{-1} \mathscr{V}_{s\left(b_{2}\right)}>\mathscr{U}_{f s\left(b_{2}\right)}$ and $g_{s\left(b_{2}\right)}^{-1} \mathscr{V}_{s\left(b_{2}\right)}>$ $\mathscr{U}_{g s\left(b_{2}\right)}$. Thus, by (3), (4), and (5),

$$
\begin{gathered}
\left(q_{b s\left(b_{2}\right)} f_{s\left(b_{2}\right)} p_{f s\left(b_{2}\right) a^{\prime}} k_{a^{\prime}} r_{k\left(a^{\prime}\right) c^{\prime}}, q_{b s\left(b_{2}\right)} f_{s\left(b_{2}\right)} k_{f s\left(b_{2}\right)} r_{k f s\left(b_{2}\right) c^{\prime}}\right)<\mathscr{V}, \\
\left(q_{b s\left(b_{2}\right)} g_{s\left(b_{2}\right)} p_{g s\left(b_{2}\right) a^{\prime}} k_{a^{\prime}} r_{k\left(a^{\prime}\right) c^{\prime}}, \quad q_{b s\left(b_{2}\right)} g_{s\left(b_{s}\right)} k_{g s\left(b_{2}\right)} r_{k g s\left(b_{2}\right) c^{\prime}}\right)<\mathscr{V}
\end{gathered}
$$

for any $c^{\prime}>c_{1}$.

By the choice of $n$, there exists a $c_{2}>c_{1}$ satisfying property (AN1) for $s(\mathbf{f k}), s(\mathbf{g k})$, and $b_{2}$. Take any $c^{\prime}>c_{2}$. By the choice of $c_{2}$, there exists a $z \in Z_{c^{\prime}}$ and a $V_{1} \in \mathrm{st}^{n} \mathscr{V}_{b_{2}}$ such that

$$
q_{b_{2} s\left(b_{2}\right)} f_{s\left(b_{2}\right)} k_{f s\left(b_{2}\right)} r_{k f s\left(b_{2}\right) c^{\prime}}(z), q_{b_{2} s\left(b_{2}\right)} g_{s\left(b_{2}\right)} k_{g s\left(b_{2}\right)} r_{k g s\left(b_{2}\right) c^{\prime}}(z) \in V_{1} .
$$

By (3) and (8), there exists a $V_{2} \in \mathscr{V}$ such that $q_{b b_{2}}\left(V_{1}\right) \subset V_{2}$, i.e.,

(9) $q_{b b_{2}} q_{b_{2} s\left(b_{2}\right)} f_{s\left(b_{2}\right)} k_{f s\left(b_{2}\right)} r_{k f s\left(b_{2}\right) c^{\prime}}(z), q_{b b_{2}} q_{b_{2} s\left(b_{2}\right)} g_{s\left(b_{2}\right)} k_{g s\left(b_{2}\right)} r_{k g s\left(b_{2}\right) c^{\prime}}(z) \in V_{2}$.

By (2) for $s\left(b_{2}\right)>b_{2}>b$, there exist $V_{3}, V_{4} \in \mathscr{V}$ such that

$$
\begin{aligned}
& q_{b b_{2}} q_{b_{2} s\left(b_{2}\right)} f_{s\left(b_{2}\right)} k_{f s\left(b_{2}\right)} r_{k f s\left(b_{2}\right) c^{\prime}}(z), \quad q_{b s\left(b_{2}\right)} f_{s\left(b_{2}\right)} k_{f s\left(b_{2}\right)} r_{k f s\left(b_{2}\right) c^{\prime}}(z) \in V_{3}, \\
& q_{b b_{2}} q_{b_{2} s\left(b_{2}\right)} g_{s\left(b_{2}\right)} k_{g s\left(b_{2}\right)} r_{k g s\left(b_{2}\right) c^{\prime}}(z), \quad q_{b s\left(b_{2}\right)} g_{s\left(b_{2}\right)} k_{g s\left(b_{2}\right)} r_{k g s\left(b_{2}\right) c^{\prime}}(z) \in V_{4} .
\end{aligned}
$$

By (6) and (7), there exist $V_{5}, V_{6} \in \mathscr{V}$ such that

$$
\begin{aligned}
& q_{b s\left(b_{2}\right)} f_{s\left(b_{2}\right)} p_{f s\left(b_{2}\right) a^{\prime}} k_{a^{\prime}} r_{k\left(a^{\prime}\right) c^{\prime}}(z), \quad q_{b s\left(b_{2}\right)} f_{s\left(b_{2}\right)} k_{f s\left(b_{2}\right)} r_{k f s\left(b_{2}\right) c^{\prime}}(z) \in V_{5}, \\
& q_{b s\left(b_{2}\right)} g_{s\left(b_{2}\right)} p_{g s\left(b_{2}\right) a^{\prime}} k_{a^{\prime}} r_{k\left(a^{\prime}\right) c^{\prime}}(z), \quad q_{b s\left(b_{2}\right)} g_{s\left(b_{2}\right)} k_{g s\left(b_{2}\right)} r_{k g s\left(b_{2}\right) c^{\prime}}(z) \in V_{6} .
\end{aligned}
$$

By (12), (10), (9), (11), (13), and (1), there exists a $V \in \mathscr{V}_{b}$ such that

$$
q_{b s\left(b_{2}\right)} f_{s\left(b_{2}\right)} p_{f s\left(b_{2}\right) a^{\prime}}(x), q_{b s\left(b_{2}\right)} g_{s\left(b_{2}\right)} p_{g s\left(b_{2}\right) a^{\prime}}(x) \in V .
$$

Here $x=k_{a^{\prime}} r_{k\left(a^{\prime}\right) c^{\prime}}(z) \in X_{c^{\prime}}$. Thus, (14) means that indices $a_{1}$ and $s\left(b_{2}\right)$ satisfy (AN) for $\mathbf{f}, \mathbf{g}$, and $b$. Hence $\mathbf{f}$ and $\mathbf{g}$ satisfy (AN).

(2.7) Corollary. Let $\mathbf{f}, \mathbf{g}:(\mathscr{X}, \mathscr{U}) \rightarrow(\mathscr{Y}, \mathscr{V})$ and $\mathbf{k}:(Z, \mathscr{W}) \rightarrow(\mathscr{X}, \mathscr{U})$ be approximate maps. If $[\mathbf{f}][\mathbf{k}]$ and $[\mathbf{g}][\mathbf{k}]$ satisfy condition $(\mathrm{AN})$, then so do [f] and $[\mathbf{g}]$.

\section{APPROXIMATE COINCIDENCE POINT}

Let $\mathscr{V} \in \operatorname{Cov}(Y)$. A point $x \in X$ is said to be a $\mathscr{V}$-coincidence point of $f$ and $g$ provided $f(x), g(x) \in V$ for some $V \in \mathscr{V}$. A point $x \in X$ is said to be a $\mathscr{V}$-fixed point of $f$ provided $x, f(x) \in V$ for some $V \in \mathscr{V}$. Let $\mathbf{p}=\left\{p_{a}: a \in A\right\}: X \rightarrow(\mathscr{X}, \mathscr{U})$ and $\mathbf{q}=\left\{q_{b}: b \in B\right\}: Y \rightarrow(\mathscr{Y}, \mathscr{V})$ be approximate polyhedral resolutions with cofinite directed sets. Let $f, g: X \rightarrow$ $Y$ be maps. Let $\mathbf{f}, \mathbf{g}:(\mathscr{X}, \mathscr{U}) \rightarrow(\mathscr{Y}, \mathscr{V})$ be approximate resolutions of $f$ and $g$ with respect to $\mathbf{p}$ and $\mathbf{q}$, respectively. 
(3.1) Lemma. f and $\mathbf{g}$ satisfy condition (AN) if and only if $f$ and $g$ have a $\mathscr{V}$-coincidence point for each $\mathscr{V} \in \operatorname{Cov}(Y)$.

Proof. First, we assume that $\mathbf{f}$ and $\mathbf{g}$ satisfy condition (AN). Take any $\mathscr{V} \in$ $\operatorname{Cov}(Y)$. By $(\mathrm{B} 1)$, there exists a $b \in B$ such that

$$
q_{b}^{-1} \mathscr{V}_{b}<\mathscr{V} \text {. }
$$

Take a $\mathscr{V}^{\prime} \in \operatorname{Cov}\left(Y_{b}\right)$ such that

$$
\text { st } \mathscr{V}^{\prime}<\mathscr{V}_{b} \text {. }
$$

By Lemma (2.2) there exist an integer $k \geq 0$ and an $a_{0}>f(b), g(b)$ satisfying (AN1) for $b \in B$. By (A3) and (AS), there exists a $b_{1}>b$ such that

$$
\begin{gathered}
q_{b b^{\prime}}^{-1} \mathscr{V}^{\prime}>\mathrm{st}^{k+3} \mathscr{V}_{b^{\prime}} \text { for any } b^{\prime}>b_{1}, \\
\left(q_{b b^{\prime}} q_{b^{\prime}}, q_{b}\right)<\mathscr{V}^{\prime} \text { for any } b^{\prime}>b_{1} .
\end{gathered}
$$

By (AS) and (A2), there exists an $a_{1}>a_{0}$ such that

$$
\begin{gathered}
\left(p_{f\left(b_{1}\right) a^{\prime}} p_{a^{\prime}}, p_{f\left(b_{1}\right)}\right)<f_{b_{1}}^{-1} \mathscr{V}_{b_{1}}, \\
\left(p_{g\left(b_{1}\right) a^{\prime}} p_{a^{\prime}}, p_{g\left(b_{1}\right)}\right)<g_{b_{1}}^{-1} \mathscr{V}_{b_{1}}
\end{gathered}
$$

for any $a^{\prime}>a_{1}$,

$$
\begin{gathered}
\left(p_{f\left(b_{1}\right) a^{\prime}} p_{a^{\prime} a^{\prime \prime}}, p_{f\left(b_{1}\right) a^{\prime \prime}}\right)<f_{b_{1}}^{-1} \mathscr{V}_{b_{1}}, \\
\left(p_{g\left(b_{1}\right) a^{\prime}} p_{a^{\prime} a^{\prime \prime}}, p_{g\left(b_{1}\right) a^{\prime \prime}}\right)<g_{b_{1}}^{-1} \mathscr{V}_{b_{1}}
\end{gathered}
$$

for $a^{\prime \prime}>a^{\prime}>a_{1}$.

Let $\mathscr{W}=\left(f_{b_{1}} p_{f\left(b_{1}\right) a_{1}}\right)^{-1} \mathscr{V}_{b_{1}} \wedge\left(g_{b_{1}} p_{g\left(b_{1}\right) a_{1}}\right)^{-1} \mathscr{V}_{b_{1}} \in \operatorname{Cov}\left(X_{a_{1}}\right)$. By (B2), there exists an $a_{2}>a_{1}$ such that

$$
p_{a_{1} a^{\prime}}\left(X_{a^{\prime}}\right) \subset \operatorname{st}\left(p_{a_{1}}(X), \mathscr{W}\right) \text { for any } a^{\prime}>a_{2} \text {. }
$$

By the choice of $a_{0}$, there exist a point $z \in X_{a_{2}}$ and a $V_{0} \in \mathrm{st}^{k} \mathscr{V}_{b_{1}}$ such that

$$
f_{b_{1}} p_{f\left(b_{1}\right) a_{2}}(z), g_{b_{1}} p_{g\left(b_{1}\right) a_{2}}(z) \in V_{0} .
$$

By (9), $p_{a_{1} a_{2}}(z) \in \operatorname{st}\left(p_{a_{1}}(X), \mathscr{W}\right)$. Thus, there exist $V_{1}, V_{2} \in \mathscr{V}_{b_{1}}$ and a point $x \in X$ such that

$$
p_{a_{1} a_{2}}(z), p_{a_{1}}(x) \in\left(f_{b_{1}} p_{f\left(b_{1}\right) a_{1}}\right)^{-1}\left(V_{1}\right) \cap\left(g_{b_{1}} p_{g\left(b_{1}\right) a_{1}}\right)^{-1}\left(V_{2}\right)
$$

By (11),

$$
\begin{aligned}
& f_{b_{1}} p_{f\left(b_{1}\right) a_{1}} p_{a_{1} a_{2}}(z), \quad f_{b_{1}} p_{f\left(b_{1}\right) a_{1}} p_{a_{1}}(x) \in V_{1}, \\
& g_{b_{1}} p_{g\left(b_{1}\right) a_{1}} p_{a_{1} a_{2}}(z), \quad g_{b_{1}} p_{g\left(b_{1}\right) a_{1}} p_{a_{1}}(x) \in V_{2} .
\end{aligned}
$$

By (5) and (6), there exist $V_{3}, V_{4} \in \mathscr{V}_{b_{1}}$ such that

$$
\begin{aligned}
& f_{b_{1}} p_{f\left(b_{1}\right) a_{1}} p_{a_{1}}(x), \quad f_{b_{1}} p_{f\left(b_{1}\right)}(x) \in V_{3}, \\
& g_{b_{1}} p_{g\left(b_{1}\right) a_{1}} p_{a_{1}}(x), \quad g_{b_{1}} p_{g\left(b_{1}\right)}(x) \in V_{4} .
\end{aligned}
$$


By (7) and (8), there exist $V_{5}, V_{6} \in \mathscr{V}_{b_{1}}$ such that

$$
\begin{aligned}
& f_{b_{1}} p_{f\left(b_{1}\right) a_{1}} p_{a_{1} a_{2}}(z), f_{b_{1}} p_{f\left(b_{1}\right) a_{2}}(z) \in V_{5}, \\
& g_{b_{1}} p_{g\left(b_{1}\right) a_{1}} p_{a_{1} a_{2}}(z), \quad g_{b_{1}} p_{g\left(b_{1}\right) a_{2}}(z) \in V_{6} .
\end{aligned}
$$

Since $\mathbf{f}$ and $\mathbf{g}$ are approximate resolutions of $f$ and $g$, respectively, by Lemma (1.15), Lemma (2.1), and (7.6) of [MW] we can assume

$$
\begin{aligned}
& \left(q_{b_{1}} f, f_{b_{1}} p_{f\left(b_{1}\right)}\right)<\mathrm{st} \mathscr{V}_{b_{1}}, \\
& \left(q_{b_{1}} g, g_{b_{1}} p_{g\left(b_{1}\right)}\right)<\mathrm{st} \mathscr{V}_{b_{1}} .
\end{aligned}
$$

By (18) and (19), there exist $V_{7}, V_{8} \in \mathrm{st} \mathscr{V}_{b_{1}}$ such that

$$
\begin{aligned}
& q_{b_{1}} f(x), \quad f_{b_{1}} p_{f\left(b_{1}\right)}(x) \in V_{7}, \\
& q_{b_{1}} g(x), \quad g_{b_{1}} p_{g\left(b_{1}\right)}(x) \in V_{8} .
\end{aligned}
$$

By (20), (14), (12), (16), (10), (17), (13), (15), and (21) there exists a $V \in$ st $^{k+3} \mathscr{V}_{b_{1}}$ such that

$$
q_{b_{1}} f(x), q_{b_{1}} g(x) \in V .
$$

By (3), there exists a $V_{1}^{\prime} \in \mathscr{V}^{\prime}$ such that

$$
q_{b b_{1}} q_{b_{1}} f(x), q_{b b_{1}} q_{b_{1}} g(x) \in V_{1}^{\prime} .
$$

By (4), there exist $V_{2}^{\prime}, V_{3}^{\prime} \in \mathscr{V}^{\prime}$ such that

$$
\begin{aligned}
& q_{b b_{1}} q_{b_{1}} f(x), q_{b} f(x) \in V_{2}^{\prime}, \\
& q_{b b_{1}} q_{b_{1}} g(x), q_{b} g(x) \in V_{3}^{\prime} .
\end{aligned}
$$

By (24), (23), and (25),

$$
q_{b} f(x), q_{b} g(x) \in \mathbf{s t}\left(V_{1}^{\prime}, \mathscr{V}^{\prime}\right) .
$$

By (1) and (2), there exist $V^{\prime} \in \mathscr{V}_{b}^{\prime}$ and $V \in \mathscr{V}$ such that $\operatorname{st}\left(V_{1}^{\prime}, \mathscr{V}^{\prime}\right) \subset V^{\prime}$ and $q_{b}^{-1} V^{\prime} \subset V$. Thus by (26),

$$
f(x), g(x) \in V .
$$

This means that $x$ is a $\mathscr{V}$-coincidence point for $f$ and $g$.

Next, we assume that $f$ and $g$ have a $\mathscr{V}$-coincidence point for any $\mathscr{V} \in$ $\operatorname{Cov}(Y)$. Take any $b \in B$. By the assumption, there exist a point $x \in X$ and a $V_{1} \in \mathscr{V}_{b}$ such that $f(x), g(x) \in q_{b}^{-1} V_{1}$, i.e.,

$$
q_{b} f(x), q_{b} g(x) \in V_{1} \text {. }
$$

Since $\mathbf{f}$ and $\mathbf{g}$ are approximate resolutions, by Lemma (1.15), Lemma (2.1), and (7.6) of [MW] we may assume (18) and (19) for any $b_{1} \in B$. Thus, there exist $V_{2}, V_{3} \in \mathrm{st} \mathscr{V}_{b}$ such that

$$
\begin{aligned}
& q_{b} f(x), \quad f_{b} p_{f(b)}(x) \in V_{2}, \\
& q_{b} g(x), \quad g_{b} p_{g(b)}(x) \in V_{3} .
\end{aligned}
$$


By (AS) for $\mathbf{p}$ there exists an $a_{0}>f(b), g(b)$ such that

$$
\begin{gathered}
\left(p_{f(b) a^{\prime}} p_{a^{\prime}}, p_{f(b)}\right)<f_{b}^{-1} \mathscr{V}_{b}, \\
\left(p_{g(b) a^{\prime}} p_{a^{\prime}}, p_{g(b)}\right)<g_{b}^{-1} \mathscr{V}_{b}
\end{gathered}
$$

for any $a^{\prime}>a_{0}$.

Take any $a^{\prime}>a_{0}$. By (31) and (32), there exist $V_{4}, V_{5} \in \mathscr{V}_{b}$ such that

$$
\begin{aligned}
& f_{b} p_{f(b) a^{\prime}} p_{a^{\prime}}(x), f_{b} p_{f(b)}(x) \in V_{4}, \\
& g_{b} p_{g(b) a^{\prime}} p_{a^{\prime}}(x), g_{b} p_{g(b)}(x) \in V_{5} .
\end{aligned}
$$

We put $z=p_{a^{\prime}}(x) \in X_{a^{\prime}}$. By (33), (29), (28), (30), and (34), there exists a $V \in \mathrm{st}^{4} \mathscr{V}_{b}$ such that $f_{b} p_{f(b) a^{\prime}}(z), g_{b} p_{g(b) a^{\prime}}(z) \in V$. Thus, $\mathbf{f}$ and $\mathbf{g}$ satisfy condition (AN1). Hence, by Lemma (2.2), they satisfy (AN).

(3.2) Corollary. $f: X \rightarrow X$ has a $\mathscr{V}$-fixed point for any $\mathscr{V} \in \operatorname{Cov}(X)$ if and only if any/some approximate resolution $\mathbf{f}:(\mathscr{X}, \mathscr{U}) \rightarrow(\mathscr{X}, \mathscr{U})$ of $f$ satisfies the following condition:

(AFP) For any $a \in A$ there exist $a_{0}>a, a_{1}>a_{0}, f\left(a_{0}\right)$ such that $p_{a a_{0}} p_{a_{0} a^{\prime}}$

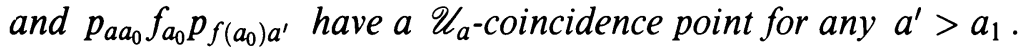

\section{Cosmicality}

An approximate map f: $\mathscr{X} \rightarrow \mathscr{Y}$ is cosmic provided it satisfies the following condition:

(AMC)

$$
\begin{aligned}
& (\forall b)\left(\exists b_{0}>b\right)\left(\exists a_{0}>f\left(b_{0}\right)\right)\left(\forall a^{\prime}>a_{0}\right)\left(\forall h: X_{a^{\prime}} \rightarrow Y_{b_{0}}\right) \\
& \left(\exists x \in X_{a^{\prime}}\right)\left(\exists V \in \mathscr{V}_{b}\right) \quad q_{b b_{0}} h(x), \quad q_{b b_{0}} f_{b_{0}} p_{f\left(b_{0}\right) a^{\prime}}(x) \in V .
\end{aligned}
$$

(4.1) Lemma. Let $\mathbf{f}, \mathbf{g}: \mathscr{X} \rightarrow \mathscr{Y}$ and $\mathbf{f} \sim \mathbf{g}$. If $\mathbf{f}$ satisfies (AMC), then so does $\mathbf{g}$.

Proof. Since $\mathbf{f} \sim \mathbf{g}$, by Lemma (7.8) of [MW] there exists an integer $k \geq 0$ with the property:

$$
\begin{aligned}
& \text { For each } b \in B \text { there exists an } a>f(b), g(b) \text { such that } \\
& \left(f_{b} p_{f(b) a^{\prime}}, g_{b} p_{g(b) a^{\prime}}\right)<\mathrm{st}^{k} \mathscr{V}_{b} \text { for any } a^{\prime}>a .
\end{aligned}
$$

Take any $b \in B$ and a $\mathscr{V} \in \operatorname{Cov}\left(Y_{b}\right)$ such that

$$
\mathbf{s t}^{2} \mathscr{V}<\mathscr{V}_{b} \text {. }
$$

By (A2) and (A3), there exists a $b_{1}>b$ such that

$$
\begin{gathered}
\left(q_{b b^{\prime}} q_{b^{\prime} b^{\prime \prime}}, q_{b b^{\prime \prime}}\right)<\mathscr{V} \text { for any } b^{\prime \prime}>b^{\prime}>b_{1}, \\
q_{b b^{\prime}}^{-1} \mathscr{V}>\mathrm{st}^{k} \mathscr{V}_{b^{\prime}} \quad \text { for any } b^{\prime}>b_{1} .
\end{gathered}
$$

Since $\mathbf{f}$ satisfies (AMC), there exist a $b_{2}>b_{1}$ and an $a_{1}>f\left(b_{1}\right)$ with the property:

For any $a^{\prime}>a_{1}$ and any map $h: X_{a^{\prime}} \rightarrow Y_{b_{2}}$ there exist an $x \in$ $X_{a^{\prime}}$ and a $V \in \mathscr{V}_{b_{1}}$ such that $q_{b_{1} b_{2}} h(x), q_{b_{1} b_{2}} f_{b_{2}} p_{f\left(b_{2}\right) a^{\prime}}(x) \in V$.

By (1) there exists an $a_{2}>f\left(b_{2}\right), g\left(b_{2}\right)$ such that

$$
\left(f_{b_{2}} p_{f\left(b_{2}\right) a^{\prime}}, g_{b_{2}} p_{g\left(b_{2}\right) a^{\prime}}\right)<\mathbf{s t}^{k} \mathscr{V}_{b_{2}}
$$

for any $a^{\prime}>a_{2}$. 
Take an $a_{3}>a_{1}, a_{2}$. Take any $a^{\prime}>a_{3}$ and any map $h: X_{a^{\prime}} \rightarrow Y_{b_{2}}$. By (5), there exist an $x \in X_{a^{\prime}}$ and a $V_{1} \in \mathscr{V}_{b_{1}}$ such that

$$
q_{b_{1} b_{2}} h(x), q_{b_{1} b_{2}} f_{b_{2}} p_{f\left(b_{2}\right) a^{\prime}}(x) \in V_{1} \text {. }
$$

Since $\left(q_{b b_{1}} q_{b_{1} b_{2}}, q_{b b_{2}}\right)<\mathscr{V}$ by (3), we have

$$
\begin{gathered}
\left(q_{b b_{1}} q_{b_{1} b_{2}} h, q_{b b_{2}} h\right)<\mathscr{V}, \\
\left(q_{b b_{1}} q_{b_{1} b_{2}} f_{b_{2}} p_{f\left(b_{2}\right) a^{\prime}}, q_{b b_{2}} f_{b_{2}} p_{f\left(b_{2}\right) a^{\prime}}\right)<\mathscr{V} .
\end{gathered}
$$

By (8) and (9), there exist $V_{2}, V_{3} \in \mathscr{V}$ such that

$$
\begin{gathered}
q_{b b_{1}} q_{b_{1} b_{2}} h(x), q_{b b_{2}} h(x) \in V_{2}, \\
q_{b b_{1}} q_{b_{1} b_{2}} f_{b_{2}} p_{f\left(b_{2}\right) a^{\prime}}(x), q_{b b_{2}} f_{b_{2}} p_{f\left(b_{2}\right) a^{\prime}}(x) \in V_{3} .
\end{gathered}
$$

Since $\left(q_{b b_{2}} f_{b_{2}} p_{f\left(b_{2}\right) a^{\prime}}, q_{b b_{2}} g_{b_{2}} p_{g\left(b_{2}\right) a^{\prime}}\right)<\mathscr{V}$ by $(4)$ and $(6)$, there exists a $V_{4} \in \mathscr{V}$ such that

$$
q_{b b_{2}} f_{b_{2}} p_{f\left(b_{2}\right) a^{\prime}}(x), q_{b b_{2}} g_{b_{2}} p_{g\left(b_{2}\right) a^{\prime}}(x) \in V_{4} .
$$

Since $q_{b b_{1}}^{-1} \mathscr{V}>\mathrm{st}^{k} \mathscr{V}_{b_{1}}>\mathscr{V}_{b_{1}}$ by (4), there exists a $V_{5} \in \mathscr{V}$ such that $q_{b b_{1}}^{-1} V_{5} \supset$ $V_{1}$. By (7) we have

$$
q_{b b_{1}} q_{b_{1} b_{2}} h(x), q_{b b_{1}} q_{b_{1} b_{2}} f_{b_{2}} p_{f\left(b_{2}\right) a^{\prime}}(x) \in V_{5} .
$$

By (10), (13), (11), (12), and (2), there exists a $V \in \mathscr{V}_{b}$ such that

$$
q_{b b_{2}} h(x), q_{b b_{2}} g_{b_{2}} p_{g\left(b_{2}\right) a^{\prime}}(x) \in V_{2} \cup V_{5} \cup V_{3} \cup V_{4} \subset V .
$$

This means that $\mathrm{g}$ satisfies (AMC).

By Lemma (4.1) we say that [f] has property (AMC) provided f has property (AMC).

(4.2) Lemma. Let $\mathbf{f}: \mathscr{X} \rightarrow \mathscr{Y}, \mathbf{g}: \mathscr{Y} \rightarrow \mathscr{Z}$, and $\mathbf{h}: \mathscr{Z} \rightarrow \mathscr{Y}$ be approximate maps. Let $[\mathbf{g}][\mathbf{h}]=\left[1_{\mathscr{Z}}\right]$. If $[\mathbf{f}]$ satisfies $(\mathrm{AMC})$, then so does $[\mathbf{g}][\mathbf{f}]$.

Proof. By (8.6) of [MW] and Lemma (4.1) we may assume that $\mathbf{f}, \mathbf{g}$, and h are uniform. Let $s: C \rightarrow C$ be a 2 -refinement function of $\mathscr{Z}$. Since $\left[1_{\mathscr{Z}}\right]=[\mathbf{g}][\mathbf{h}]=[s(\mathbf{g h})]$, by Lemma $(7.8)$ of $[\mathrm{MW}]$, there exists an integer $k \geq 0$ with the property: for each $c \in C$ there exists a $c^{\prime}>s(c)$, hgs $(c)$ such that

$$
\left(r_{c c^{\prime \prime}}, r_{c, s(c)} g_{s(c)} h_{g s(c)} r_{h s(c), c^{\prime \prime}}\right)<\mathrm{st}^{k} \mathscr{W}_{c}
$$

for any $c^{\prime \prime}>c^{\prime}$.

Take any $c \in C$ and any $\mathscr{W} \in \operatorname{Cov}\left(Z_{c}\right)$ such that

$$
\mathrm{st}^{2} \mathscr{W}<\mathscr{W}_{c} \text {. }
$$

By (A2) and (A3), there exists a $c_{1}>c$ such that

$$
\begin{gathered}
r_{c c^{\prime}}^{-1} \mathscr{W}>s^{k+2} \mathscr{W}_{c^{\prime}} \quad \text { for any } c^{\prime}>c_{1}, \\
\left(r_{c c^{\prime}} r_{c^{\prime} c^{\prime \prime}}, r_{c c^{\prime \prime}}\right)<\mathscr{W} \quad \text { for any } c^{\prime \prime}>c^{\prime}>c_{1} .
\end{gathered}
$$

By (1), there exists a $c_{2}>s\left(c_{1}\right), h g s\left(c_{1}\right)$ such that

$$
\left(r_{c_{1} c^{\prime}}, r_{c_{1}, s\left(c_{1}\right)} g_{s\left(c_{1}\right)} h_{g s\left(c_{1}\right)} r_{h g s\left(c_{1}\right), c^{\prime}}\right)<\mathbf{s t}^{k} \mathscr{W}_{c_{1}} \quad \text { for any } c^{\prime}>c_{2} \text {. }
$$


Since $\mathbf{f}$ satisfies (AMC), there exist a $b_{1}>g s\left(c_{1}\right)$ and an $a_{1}>f\left(b_{1}\right)$ satisfying the condition (AMC) for $\mathbf{f}$ and $g s\left(c_{1}\right)$. By (AM) there exists a $c_{3}>h\left(b_{1}\right), c_{2}$ such that

(6) $\quad\left(q_{g s\left(c_{1}\right), h_{1}} h_{b_{1}} r_{h\left(b_{1}\right), c^{\prime}}, h_{g s\left(c_{1}\right)} r_{h g s\left(c_{1}\right), c^{\prime}}\right)<s t \mathscr{V}_{g s\left(c_{1}\right)}$ for any $c^{\prime}>c_{3}$.

By (AM), there exists an $a_{2}>f\left(b_{1}\right), f g s\left(c_{1}\right)$ such that

(7) $\quad\left(q_{g s\left(c_{1}\right), b_{1}} f_{b_{1}} p_{f\left(b_{1}\right), a^{\prime}}, f_{g s\left(c_{1}\right)} p_{f g s\left(c_{1}\right), a^{\prime}}\right)<$ st $\mathscr{V}_{g s\left(c_{1}\right)} \quad$ for any $a^{\prime}>a_{2}$.

By (AM) for $s(\mathbf{g f})$, there exists an $a_{3}>a_{2}, f g s\left(c_{3}\right)$ such that

(8) $\quad\left(r_{c_{1} c_{3}} r_{c_{3}, s\left(c_{3}\right)} g_{s\left(c_{3}\right)} f_{g s\left(c_{3}\right)} p_{f g s\left(c_{3}\right), a^{\prime}}, r_{c_{1}, s\left(c_{1}\right)} g_{s\left(c_{1}\right)} f_{g s\left(c_{1}\right)} p_{f g s\left(c_{1}\right), a^{\prime}}\right)<s t \mathscr{W}_{c_{1}}$

for any $a^{\prime}>a_{3}$.

We show that $s$ (gf) satisfies (AMC), i.e., indices $a_{3}$ and $c_{3}$ are the required ones. To do so, take any $a^{\prime}>a_{3}$ and any map $\alpha: X_{a^{\prime}} \rightarrow Z_{c_{3}}$. By the choice of $a_{1}$, there exist a point $x \in X_{a^{\prime}}$ and a $V_{0} \in \mathscr{V}_{g s\left(c_{1}\right)}$ such that

$$
q_{g s\left(c_{1}\right), b_{1}} f_{b_{1}} p_{f\left(b_{1}\right) a^{\prime}}(x), q_{g s\left(c_{1}\right), b_{1}} h_{b_{1}} r_{h\left(b_{1}\right), c_{3}} \alpha(x) \in V_{0} \text {. }
$$

Since $\mathbf{g}$ is uniform, there exists a $W_{0} \in \mathscr{W}_{c_{1}}$ such that $g_{s\left(c_{1}\right)}^{-1} r_{c_{1} s\left(c_{1}\right)}^{-1}\left(W_{0}\right) \supset V_{0}$. Then, by (9),

$$
r_{c_{1}, s\left(c_{1}\right)} g_{s\left(c_{1}\right)} q_{g s\left(c_{1}\right), b_{1}} f_{b_{1}} p_{f\left(b_{1}\right), a^{\prime}}(x), r_{c_{1}, s\left(c_{1}\right)} g_{s\left(c_{1}\right)} q_{g s\left(c_{1}\right), b_{1}} h_{b_{1}} r_{h\left(b_{1}\right), c_{3}} \alpha(x) \in W_{0}
$$

Since $\mathbf{g}$ is uniform and $s$ is a 2-refinement function, (5), (6), and (7) imply that

$$
\begin{gathered}
\left(r_{c_{1} c_{3}} \alpha, r_{c_{1} s\left(c_{1}\right)} g_{s\left(c_{1}\right)} h_{g s\left(c_{1}\right)} r_{h g s\left(c_{1}\right), c_{3}} \alpha\right)<s^{k} \mathscr{W}_{c_{1}}, \\
\left(r_{c_{1} s\left(c_{1}\right)} g_{s\left(c_{1}\right)} q_{g s\left(c_{1}\right), b_{1}} h_{b_{1}} r_{h\left(b_{1}\right), c_{3}} \alpha, r_{c_{1} s\left(c_{1}\right)} g_{s\left(c_{1}\right)} h_{g s\left(c_{1}\right)} r_{h g s\left(c_{1}\right), c_{3}} \alpha\right)<\mathscr{W}_{c_{1}}, \\
\left(r_{c_{1}, s\left(c_{1}\right)} g_{s\left(c_{1}\right)} q_{g s\left(c_{1}\right), b_{1}} f_{b_{1}} p_{f\left(b_{1}\right) a^{\prime}}, r_{c_{1}, s\left(c_{1}\right)} g_{s\left(c_{1}\right)} f_{g s\left(c_{1}\right)} p_{f g s\left(c_{1}\right), a^{\prime}}\right)<\mathscr{W}_{c_{1}} .
\end{gathered}
$$

By (11), (12), (10), and (13), there exists a $W_{1} \in \mathrm{st}^{k+2} \mathscr{W}_{c_{1}}$ such that

(14) $r_{c_{1} c_{3}} \alpha(x), r_{c_{1} s\left(c_{1}\right)} g_{s\left(c_{1}\right)} f_{g s\left(c_{1}\right)} p_{f g s\left(c_{1}\right) a^{\prime}}(x) \in \mathbf{s t}\left(\mathbf{s t}\left(W_{0}, \mathscr{W}_{c_{1}}\right), \mathbf{s t}^{k} \mathscr{W}_{c_{1}}\right) \subset W_{1}$.

By (3) and (14), there exists a $W_{2} \in \mathscr{W}$ such that

$$
r_{c c_{1}} r_{c_{1} c_{3}} \alpha(x), r_{c c_{1}} r_{c_{1} s\left(c_{1}\right)} g_{s\left(c_{1}\right)} f_{g s\left(c_{1}\right)} p_{f g s\left(c_{1}\right), a^{\prime}}(x) \in r_{c c_{1}}\left(W_{1}\right) \subset W_{2} .
$$

By (4) there exist $W_{3}, W_{4} \in \mathscr{W}$ such that

$$
\begin{gathered}
r_{c c_{1}} r_{c_{1} c_{3}} \alpha(x), r_{c c_{3}} \alpha(x) \in W_{3}, \\
r_{c c_{1}} r_{c_{1} c_{3}} r_{c_{3}, s\left(c_{3}\right)} g_{s\left(c_{3}\right)} f_{g s\left(c_{3}\right)} p_{f g s\left(c_{3}\right), a^{\prime}}(x), \\
r_{c c_{3}} r_{c_{3}, s\left(c_{3}\right)} g_{s\left(c_{3}\right)} f_{g s\left(c_{3}\right)} p_{f g s\left(c_{3}\right), a^{\prime}}(x) \in W_{4} .
\end{gathered}
$$

By (8) and (3), there exists a $W_{5} \in \mathscr{W}$ such that

$$
\begin{aligned}
& r_{c c_{1}} r_{c_{1} c_{3}} r_{c_{3}, s\left(c_{3}\right)} g_{s\left(c_{3}\right)} f_{g s\left(c_{3}\right)} p_{f g s\left(c_{3}\right), a^{\prime}}(x), \\
& r_{c c_{1}} r_{c_{1}, s\left(c_{1}\right)} g_{s\left(c_{1}\right)} f_{g s\left(c_{1}\right)} p_{f g s\left(c_{1}\right), a^{\prime}}(x) \in W_{5} .
\end{aligned}
$$

By (16), (15), (17), (18), and (2), there exists a $W_{6} \in \mathscr{W}_{c}$ such that

$$
\begin{aligned}
& r_{c c_{3}} r_{c_{3}, s\left(c_{3}\right)} g_{s\left(c_{3}\right)} f_{g s\left(c_{3}\right)} p_{f g s\left(c_{3}\right), a^{\prime}}(x), \\
& r_{c c_{3}} \alpha(x) \in W_{4} \cup W_{3} \subset \operatorname{st}\left(\operatorname{st}\left(W_{2}, \mathscr{W}\right), \mathrm{st} \mathscr{W}\right) \subset W_{6} .
\end{aligned}
$$

This means that $s(\mathbf{g f})$ satisfies $(\mathrm{AMC})$. Hence $[s(\mathbf{g f})]=[\mathbf{g}][\mathbf{f}]$ has (AMC).

The proof of the next lemma is similar to the proof of Lemma (4.2). 
(4.3) Lemma. Let $\mathbf{f}: \mathscr{X} \rightarrow \mathscr{Y}, \mathbf{g}: \mathscr{Y} \rightarrow \mathscr{Z}$, and $\mathbf{u}=\left\{u, u_{a}: a \in A\right\}: \mathscr{Y} \rightarrow$ $\mathscr{Z}$ be approximate maps. Let $[\mathbf{f}][\mathbf{u}]=\left[1_{\mathscr{y}}\right]$. If $[\mathbf{g}]$ has (AMC), then so does [g][f].

(4.4) Lemma. Let $\mathbf{f}: \mathscr{Z} \rightarrow \mathscr{Y}$ and $\mathbf{g}: \mathscr{Y} \rightarrow \mathscr{Z}$ be approximate maps. If [g][f] satisfies (AMC), then so does [g].

Proof. We may assume that $\mathbf{f}$ and $\mathbf{g}$ are uniform. Let $w: C \rightarrow C$ be a 2-refinement function for $\mathscr{Z}$. Take any $c \in C$ and a $\mathscr{W} \in \operatorname{Cov}\left(Z_{c}\right)$ such that

$$
\mathrm{st}^{2} \mathscr{W}<\mathscr{W}_{c} \text {. }
$$

By (A2) and (A3), there exists a $c_{1}>c$ such that

$$
\begin{gathered}
\left(r_{c c^{\prime \prime}}, r_{c c^{\prime}} r_{c^{\prime} c^{\prime \prime}}\right)<\mathscr{W} \quad \text { for any } c^{\prime \prime}>c^{\prime}>c_{1}, \\
r_{c c^{\prime}}^{-1} \mathscr{W}>\mathscr{W}_{c^{\prime}} \quad \text { for any } c^{\prime}>c_{1} .
\end{gathered}
$$

By the assumption, $w\left(\right.$ gf) satisfies (AMC). Then, there exist indices $c_{2}>c_{1}$ and $a_{1}>f g w\left(c_{2}\right)$ satisfying the condition (AMC) for $w$ (gf) and $c_{1}$.

We show that $\mathrm{g}$ satisfies (AMC), i.e., we show the indices $w\left(c_{2}\right)>c$ and $g w\left(c_{2}\right)>g w\left(c_{2}\right)$ satisfy the condition (AMC) for $\mathbf{g}$ and $c$. Take any $b^{\prime}>$ $g w\left(c_{2}\right)$ and any map $\alpha: Y_{b^{\prime}} \rightarrow Z_{w\left(c_{2}\right)}$. By (AM) for $\mathbf{f}$, there exists an $a_{2}>a_{1}$, $f\left(b^{\prime}\right)$ such that

(4) $\left(f_{g w\left(c_{2}\right)} p_{f g w\left(c_{2}\right) a^{\prime}}, q_{g w\left(c_{2}\right), b^{\prime}} f_{b^{\prime}} p_{f\left(b^{\prime}\right) a^{\prime}}\right)<\mathrm{st} \mathscr{V}_{g w\left(c_{2}\right)}$ for any $a^{\prime}>a_{2}$.

By the choice of $a_{1}$ and $c_{2}$, there exist a point $x \in X_{a_{2}}$ and a $W_{1} \in \mathscr{W}_{c_{1}}$ such that

(5) $\quad r_{c_{1} c_{2}} r_{c_{2} w\left(c_{2}\right)} g_{w\left(c_{2}\right)} f_{g w\left(c_{2}\right)} p_{f g w\left(c_{2}\right) a_{2}}(x), r_{c_{1} c_{2}} r_{c_{2} w\left(c_{2}\right)} \alpha f_{b^{\prime}} p_{f\left(b^{\prime}\right) a_{2}}(x) \in W_{1}$.

By (5) and (3), there exists a $W_{2} \in \mathscr{W}$ such that

$$
\begin{aligned}
& r_{c c_{1}} r_{c_{1} c_{2}} r_{c_{2} w\left(c_{2}\right)} g_{w\left(c_{2}\right)} f_{g w\left(c_{2}\right)} p_{f g w\left(c_{2}\right) a_{2}}(x), \\
& r_{c c_{1}} r_{c_{1} c_{2}} r_{c_{2} w\left(c_{2}\right)} \alpha f_{b^{\prime}} p_{f\left(b^{\prime}\right) a_{2}}(x) \in r_{c c_{1}}\left(W_{1}\right) \subset W_{2} .
\end{aligned}
$$

By (2),

$$
\begin{gathered}
\left(r_{c c_{2}} r_{c_{2} w\left(c_{2}\right)}, r_{c w\left(c_{2}\right)}\right)<\mathscr{W}, \\
\left(r_{c c_{1}} r_{c_{1} c_{2}} r_{c_{2} w\left(c_{2}\right)}, r_{c c_{2}} r_{c_{2} w\left(c_{2}\right)}\right)<\mathscr{W} .
\end{gathered}
$$

By (8), there exist $W_{3}, W_{4} \in \mathscr{W}$ such that

$$
\begin{gathered}
r_{c c_{1}} r_{c_{1} c_{2}} r_{c_{2} w\left(c_{2}\right)} g_{w\left(c_{2}\right)} f_{g w\left(c_{2}\right)} p_{f g w\left(c_{2}\right) a_{2}}(x), \\
r_{c c_{2}} r_{c_{2} w\left(c_{2}\right)} g_{w\left(c_{2}\right)} f_{g w\left(c_{2}\right)} p_{f g w\left(c_{2}\right) a_{2}}(x) \in W_{3}, \\
r_{c c_{1}} r_{c_{1} c_{2}} r_{c_{2} w\left(c_{2}\right)} \alpha f_{b^{\prime}} p_{f\left(b^{\prime}\right) a_{2}}(x), r_{c c_{2}} r_{c_{2} w\left(c_{2}\right)} \alpha f_{b^{\prime}} p_{f\left(b^{\prime}\right) a_{2}}(x) \in W_{4} .
\end{gathered}
$$

By (7), there exist $W_{5}, W_{6} \in \mathscr{W}$ such that

$$
\begin{gathered}
r_{c c_{2}} r_{c_{2} w\left(c_{2}\right)} g_{w\left(c_{2}\right)} f_{g w\left(c_{2}\right)} p_{f g w\left(c_{2}\right) a_{2}}(x), \\
r_{c w\left(c_{2}\right)} g_{w\left(c_{2}\right)} f_{g w\left(c_{2}\right)} p_{f g w\left(c_{2}\right) a_{2}}(x) \in W_{5}, \\
r_{c c_{2}} r_{c_{2} w\left(c_{2}\right)} \alpha f_{b^{\prime}} p_{f\left(b^{\prime}\right) a_{2}}(x), r_{c w\left(c_{2}\right)} \alpha f_{b^{\prime}} p_{f\left(b^{\prime}\right) a_{2}}(x) \in W_{6} .
\end{gathered}
$$


Since $\mathbf{g}$ is uniform, by $(3) g_{w\left(c_{2}\right)}^{-1} r_{c w\left(c_{2}\right)}^{-1} \mathscr{W}>\mathscr{V}_{g w\left(c_{2}\right)}$. Then, by (4),

$$
\left(r_{c w\left(c_{2}\right)} g_{w\left(c_{2}\right)} f_{g w\left(c_{2}\right)} p_{f g w\left(c_{2}\right) a_{2}}, \quad r_{c w\left(c_{2}\right)} g_{w\left(c_{2}\right)} q_{g w\left(c_{2}\right), b^{\prime}} f_{b^{\prime}} p_{f\left(b^{\prime}\right) a_{2}}\right)<\mathrm{st} \mathscr{W} .
$$

By (13), there exists a $W_{7} \in$ st $\mathscr{W}$ such that

$$
r_{c w\left(c_{2}\right)} g_{w\left(c_{2}\right)} f_{g w\left(c_{2}\right)} p_{f g w\left(c_{2}\right) a_{2}}(x), r_{c w\left(c_{2}\right)} g_{w\left(c_{2}\right)} q_{g w\left(c_{2}\right) b^{\prime}} f_{b^{\prime}} p_{f\left(b^{\prime}\right) a_{2}}(x) \in W_{7}
$$

By (12), (10), (6), (9), (11), (14), and (1), there exists a $W \in \mathscr{W}_{c}$ such that

$$
\begin{gathered}
r_{c w\left(c_{2}\right)} \alpha(y), r_{c w\left(c_{2}\right)} g_{w\left(c_{2}\right)} q_{g w\left(c_{2}\right) b^{\prime}}(y) \in W_{6} \cup W_{4} \cup W_{2} \cup W_{3} \cup W_{5} \cup W_{7} \\
\subset \operatorname{st}\left(\operatorname{st}\left(W_{3}, \mathscr{W}\right), \text { st } \mathscr{W}\right) \subset W .
\end{gathered}
$$

Here $y=f_{b^{\prime}} p_{f\left(b^{\prime}\right) a_{2}}(x) \in Y_{b^{\prime}}$. Therefore, (15) means that g satisfies (AMC). Hence [g] satisfies (AMC).

The proof of the following lemma is similar to the proof of Lemma (4.2).

(4.5) Lemma. Let $\mathbf{f}: \mathscr{X} \rightarrow \mathscr{Y}, \mathbf{g}: \mathscr{Y} \rightarrow \mathscr{Z}$, and $\mathbf{h}: \mathscr{Z} \rightarrow \mathscr{Y}$ be approximate maps. Let $[\mathbf{h}][\mathbf{g}]=\left[1_{y}\right]$. If $[\mathbf{g}][\mathbf{f}]$ satisfies $(\mathrm{AMC})$, then so does [f] .

Let $\mathbf{p}^{\prime}: X \rightarrow \mathscr{X}^{\prime}$ and $\mathbf{q}^{\prime}: Y \rightarrow \mathscr{Y}^{\prime}$ be cofinite approximate APOLresolutions. Let $\mathbf{f}^{\prime}: \mathscr{X}^{\prime} \rightarrow \mathscr{Y}^{\prime}$ be an approximate resolution of $f$.

(4.6) Lemma. Let ( $\mathbf{p}, \mathbf{q}, \mathbf{f})$ and $\left(\mathbf{p}^{\prime}, \mathbf{q}^{\prime}, \mathbf{f}^{\prime}\right)$ be approximate resolutions of $f$.

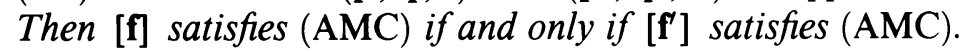

Proof. By (6.3) of [MW] there exist approximate resolutions (p, $\left.\mathbf{p}^{\prime}, \mathbf{u}\right)$, $\left(\mathbf{q}, \mathbf{q}^{\prime}, \mathbf{v}\right)$, and $\left(\mathbf{p}, \mathbf{q}^{\prime}, \mathbf{h}\right)$ of $1_{X}, 1_{Y}$, and $f$, respectively. Then by Corollary (1.17),

$$
[\mathbf{v}][\mathbf{f}]=[\mathbf{h}]=\left[\mathbf{f}^{\prime}\right][\mathbf{u}] .
$$

We assume that [f] satisfies (AMC). Since [v] is an isomorphism by Corollary

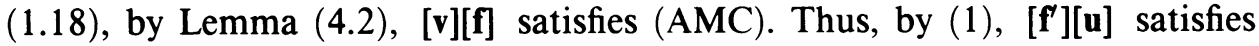
(AMC). Hence by Lemma (4.4), [f'] satisfies (AMC).

Similarly we can show the converse. Hence, we have Lemma (4.6).

By Lemma (4.6), we say a map $f: X \rightarrow Y$ satisfies (AMC) provided some/any approximate APOL-resolution $\mathbf{f}: \mathscr{X} \rightarrow \mathscr{Y}$ of $f$ satisfies (AMC).

(4.7) Lemma. If $f: X \rightarrow Y$ satisfies (AMC), then for any map $g: X \rightarrow Y$ and for any $\mathscr{V} \in \operatorname{Cov}(Y), f$ and $g$ have a $\mathscr{V}$-coincidence point.

Proof. Take any map $g: X \rightarrow Y$. Let $\mathbf{p}: X \rightarrow \mathscr{X}$ and $\mathbf{q}: Y \rightarrow \mathscr{Y}$ be cofinite APOL-resolutions. Let $\mathbf{f}: \mathscr{X} \rightarrow \mathscr{Y}$ and $\mathbf{g}: \mathscr{X} \rightarrow \mathscr{Y}$ be approximate resolutions of $f$ and $g$, respectively.

We show that $\mathbf{f}$ and $\mathbf{g}$ satisfy condition (AN). By the assumption on $f, \mathbf{f}$ satisfies (AMC). Take any $b \in B$. Thus there exist a $b_{0}>b$ and an $a_{0}>f\left(b_{0}\right)$ satisfying condition (AMC). Take an $a_{1}>a_{0}, g\left(b_{0}\right)$. We easily show that the indices $a_{1}$ and $b_{0}$ satisfy condition (AN). Thus $\mathbf{f}$ and $g$ satisfy (AN). Hence, by (3.1), $f$ and $g$ have a $\mathscr{V}$-coincidence point for any $\mathscr{V} \in \operatorname{Cov}(Y)$.

(4.8) Lemma. Let $Y$ be an approximate polyhedron, and let $f: X \rightarrow Y$ be a map. Then $f$ satisfies (AMC) if and only if 
(i) for any map $g: X \rightarrow Y$ and any $\mathscr{V} \in \operatorname{Cov}(Y), f$ and $g$ have $a \mathscr{V}$. coincidence point.

Proof. Let $B$ be the set of all nonempty finite sets $b=\left\{\mathscr{V}_{1}, \ldots, \mathscr{V}_{n}\right\}, \mathscr{V}_{i} \in$ $\operatorname{Cov}(Y)$. An order $<$ in $B$ is defined as follows: $b<b^{\prime}$ provided $b \subset b^{\prime}$. Thus $(B,<)$ is a cofinite directed ordered set.

For each $b=\left\{\mathscr{V}_{1}, \ldots, \mathscr{V}_{n}\right\}$, let $\mathscr{V}_{b}=\mathscr{V}_{1} \wedge \cdots \wedge \mathscr{V}_{n}=\left\{V_{1} \cap \cdots \cap V_{n}\right.$ : $\left.V_{i} \in \mathscr{V}_{i}, i=1,2, \ldots, n\right\} \in \operatorname{Cov}(Y), Y_{b}=Y, q_{b}=1_{Y}: Y \rightarrow Y_{b}$, and $q_{b b^{\prime}}=1_{Y}: Y_{b^{\prime}}=Y \rightarrow Y=Y_{b}$ for $b<b^{\prime}$. It is easy to show that $\mathbf{q}=$ $\left\{q_{b}: b \in B\right\}: Y \rightarrow \mathscr{Y}=\left\{Y_{b}, \mathscr{V}_{b}, q_{b b^{\prime}}, B\right\}$ satisfies (B1) and (B2). Hence, by (2.8) of [MW], $\mathbf{q}$ is an approximate APOL-resolution of $Y$. This is called the rudimentary (approximate) resolution of $Y$.

Let $\mathbf{p}: X \rightarrow \mathscr{X}$ be a cofinite approximate APOL-resolution and $\mathbf{f}: \mathscr{X} \rightarrow \mathscr{Y}$ be an approximate resolution of $f$. By Lemma (1.15), we may assume that

$$
\left(f_{b} p_{f(b)}, q_{b} f\right)<\mathrm{st} \mathscr{V}_{b} \text { for } b \in B .
$$

We show that (i) implies (AMC) for $\mathbf{f}$. To do so take any $b=\left\{\mathscr{V}_{1}, \ldots, \mathscr{V}_{n}\right\}$ $\in B$. Take a $\mathscr{V} \in \operatorname{Cov}(Y)$ such that

$$
\mathrm{st}^{2} \mathscr{V}<\mathscr{V}_{b}
$$

Let $b_{0}=\left\{\mathscr{V}_{1}, \ldots, \mathscr{V}_{n}, \mathscr{V}\right\} \in B$. By (AS) for $\mathbf{p}$, there exists $a_{0}>f\left(b_{0}\right)$ such that

$$
\left(p_{f\left(b_{0}\right)}, p_{f\left(b_{0}\right) a^{\prime}} p_{a^{\prime}}\right)<f_{b_{0}}^{-1}\left(\mathscr{V}_{b_{0}}\right)
$$

for any $a^{\prime}>a_{0}$.

We show that the indices $a_{0}$ and $b_{0}$ satisfy property (AMC) for $\mathbf{f}$. Take any $a^{\prime}>a_{0}$ and any map $h: X_{a^{\prime}} \rightarrow Y_{b_{0}}$. By the assumption (i), there exist a point $x \in X$ and a $V_{1} \in \mathscr{V}_{b_{0}}$ such that

$$
f(x), h p_{a^{\prime}}(x) \in V_{1} .
$$

By (1) for $b_{0}$, there exists a $V_{2} \in$ st $\mathscr{V}_{b_{0}}$ such that

$$
f_{b_{0}} p_{f\left(b_{0}\right)}(x), q_{b_{0}} f(x) \in V_{2} \text {. }
$$

By (3), there exists a $V_{3} \in \mathscr{V}_{b_{0}}$ such that

$$
f_{b_{0}} p_{f\left(b_{0}\right)}(x), f_{b_{0}} p_{f\left(b_{0}\right) a^{\prime}} p_{a^{\prime}}(x) \in V_{3} .
$$

Since $q_{b_{0}}=1_{Y}$, by (4), (5), and (6)

$$
h p_{a^{\prime}}(x), f_{b_{0}} p_{f\left(b_{0}\right) a^{\prime}} p_{a^{\prime}}(x) \in \operatorname{st}\left(V_{2}, \text { st } \mathscr{V}_{b_{0}}\right) .
$$

Since $q_{b b_{0}}=1_{Y}$, by (2) and the choice of $b_{0}, q_{b b_{0}}^{-1} \mathscr{V}_{b}=\mathscr{V}_{b}>\mathbf{s t}^{2} \mathscr{V}>$ $\operatorname{st}^{2}\left(\mathscr{V}_{1} \wedge \cdots \wedge \mathscr{V}_{n} \wedge \mathscr{V}\right)=\mathrm{st}^{2} \mathscr{V}_{b_{0}}$. Thus, by (7), there exists a $V_{4} \in \mathscr{V}_{b}$ such that

$$
q_{b b_{0}} h\left(x^{\prime}\right), q_{b b_{0}} f_{b_{0}} p_{f\left(b_{0}\right) a^{\prime}}\left(x^{\prime}\right) \in q_{b b_{0}}\left(\operatorname{st}\left(V_{2}, \text { st } \mathscr{V}_{b_{0}}\right)\right) \subset V_{4} .
$$

Here $x^{\prime}=p_{a^{\prime}}(x) \in X_{a^{\prime}}$. (8) means that $\mathbf{f}$ satisfies (AMC). Hence $f$ satisfies (AMC).

The converse assertion follows from Lemma (4.7).

\section{COSMIC SYSTEMS AND RESOLUTIONS}

In this section we generalize the notion of universality due to Holsztynski [H] and Mioduszewski and Rochowski [MiR] to approximate systems and resolutions. It should be noted that the version of universality we describe (condition 
$(\mathrm{AC}))$ is more than just a generalization of the previous notion to approximate systems. We not only show that the limit of an ANR-resolution satisfying condition (AC) has the fixed point property (5.12), but that condition (AC) also is an approximate shape invariant (see (5.3)). In other words, condition (AC) is categorical and hence does not depend on the particular expansion chosen. This is in contradistinction to the notion of universality due to Holsztynski (see Example (5.7)).

(5.1) Lemma. The following conditions are equivalent.

(i) $\left[1_{\mathscr{Z}}\right]: \mathscr{X} \rightarrow \mathscr{X}$ satisfies (AMC).

(ii) $\mathscr{Z}$ satisfies condition (AC):

$$
\begin{aligned}
& (\forall a \in A)\left(\exists a_{0}>a\right)\left(\forall a^{\prime}>a_{0}\right)\left(\forall h: X_{a^{\prime}} \rightarrow X_{a_{0}}\right) \\
& \left(\exists x \in X_{a^{\prime}}\right)\left(\exists U \in \mathscr{U}_{a}\right) p_{a a_{0}} h(x), p_{a a_{0}} p_{a_{0} a^{\prime}}(x) \in U .
\end{aligned}
$$

(iii) $\mathscr{X}$ satisfies condition (AC1):

$$
\begin{aligned}
& (\forall a \in A)\left(\forall \mathscr{U} \in \operatorname{Cov}\left(X_{a}\right)\right)\left(\exists a_{0}>a\right)\left(\forall a^{\prime}>a_{0}\right)\left(\forall h: X_{a^{\prime}} \rightarrow X_{a_{0}}\right) \\
& \left(\exists x_{0} \in X_{a^{\prime}}\right)(\exists U \in \mathscr{U}) p_{a a_{0}} h\left(x_{0}\right), p_{a a_{0}} p_{a_{0} a^{\prime}}\left(x_{0}\right) \in U .
\end{aligned}
$$

Proof. We first show that (i) implies (ii). Take any $a \in A$ and a $\mathscr{U} \in \operatorname{Cov}\left(X_{a}\right)$ such that

$$
\mathrm{st}^{2} \mathscr{U}<\mathscr{U}_{a} \text {. }
$$

By (A2) and (A3), there exists an $a_{0}>a$ such that

$$
\begin{gathered}
\left(p_{a a^{\prime \prime}}, p_{a a^{\prime}} p_{a^{\prime} a^{\prime \prime}}\right)<\mathscr{U} \text { for any } a^{\prime \prime}>a^{\prime}>a_{0}, \\
p_{a a^{\prime}}^{-1} \mathscr{U}>\mathscr{U}_{a^{\prime}} \quad \text { for any } a^{\prime}>a_{0} .
\end{gathered}
$$

By (i), there exist indices $a_{2}>a_{1}>a_{0}$ satisfying the condition (AMC) for $1_{\mathscr{Z}}$ and $a_{0}$.

We show that the index $a_{2}$ satisfies (AC) for $a$. To do so take any $a^{\prime}>a_{2}$ and any map $h: X_{a^{\prime}} \rightarrow X_{a_{2}}$. Since $p_{a_{1} a_{2}} h: X_{a^{\prime}} \rightarrow X_{a_{1}}$, by the choices of $a_{1}$ and $a_{2}$ there exist an $x \in X_{a^{\prime}}$ and a $U_{1} \in \mathscr{U}_{a_{0}}$ such that

$$
p_{a_{0} a_{1}} p_{a_{1} a_{2}} h(x), p_{a_{0} a_{1}} p_{a_{1} a^{\prime}}(x) \in U_{1} \text {. }
$$

By (3), there exists a $U_{2} \in \mathscr{U}$ such that $p_{a a_{0}}^{-1} U_{2} \supset U_{1}$. Thus, by (4),

$$
p_{a a_{0}} p_{a_{0} a_{1}} p_{a_{1} a_{2}} h(x), p_{a a_{0}} p_{a_{0} a_{1}} p_{a_{1} a^{\prime}}(x) \in p_{a a_{0}}\left(U_{1}\right) \subset U_{2} \text {. }
$$

Since $\left(p_{a a_{0}} p_{a_{0} a_{1}}, p_{a a_{1}}\right)<\mathscr{U}$ by $(2)$,

$$
\begin{gathered}
\left(p_{a a_{0}} p_{a_{0} a_{1}} p_{a_{1} a_{2}} h, p_{a a_{1}} p_{a_{1} a_{2}} h\right)<\mathscr{U}, \\
\left(p_{a a_{0}} p_{a_{0} a_{1}} p_{a_{1} a^{\prime}}, p_{a a_{1}} p_{a_{1} a^{\prime}}\right)<\mathscr{U} .
\end{gathered}
$$

Since $\left(p_{a a_{1}} p_{a_{1} a_{2}}, p_{a a_{2}}\right)<\mathscr{U}$ by $(2)$

$$
\begin{gathered}
\left(p_{a a_{1}} p_{a_{1} a_{2}} p_{a_{2} a^{\prime}}, \quad p_{a a_{2}} p_{a_{2} a^{\prime}}\right)<\mathscr{U}, \\
\left(p_{a a_{1}} p_{a_{1} a_{2}} h, \quad p_{a a_{2}} h\right)<\mathscr{U} .
\end{gathered}
$$

Since $\left(p_{a_{1} a_{2}} p_{a_{2} a^{\prime}}, p_{a_{1} a^{\prime}}\right)<\mathscr{U}_{a_{1}}$ by $(\mathrm{A} 1)$ and $p_{a a_{1}}^{-1} \mathscr{U}>\mathscr{U}_{a_{1}}$ by (3),

$$
\left(p_{a a_{1}} p_{a_{1} a_{2}} p_{a_{2} a^{\prime}}, p_{a a_{1}} p_{a_{1} a^{\prime}}\right)<\mathscr{U} \text {. }
$$


By (9), (6), (5), (7), (10), (8), and (1), there exists a $U \in \mathscr{U}_{a}$ such that

$$
p_{a a_{2}} h(x), p_{a a_{2}} p_{a_{2} a^{\prime}}(x) \in \operatorname{st}\left(\operatorname{st}\left(U_{2}, \mathscr{U}\right), \text { st } \mathscr{U}\right) \subset U .
$$

This means that $a_{2}$ satisfies the condition (AC) for $a$. Thus $\mathscr{X}$ satisfies (AC).

We show that (ii) implies (i). Take any $a \in A$. By (ii), there exists an $a_{0}>a$ satisfying (AC) for $\mathscr{X}$ and $a$. We show that the indices $a_{0}>1_{A}\left(a_{0}\right)$ and $a_{0}>a$ satisfy (AMC) for $1_{\mathscr{L}}$ and $a$. To do so take any $a^{\prime}>a_{0}$ and any map $h: X_{a^{\prime}} \rightarrow X_{a_{0}}$. By the choice of $a_{0}$, there exist an $x \in X_{a^{\prime}}$ and a $U \in \mathscr{U}_{a}$ such that $p_{a a_{0}} h(x), p_{a a_{0}} p_{a_{0} a^{\prime}}(x) \in U$. Since $p_{a a_{0}} p_{a_{0} a^{\prime}}=p_{a a_{0}} 1_{X_{a_{0}}} p_{a_{0} a^{\prime}}$, this means that $1_{\mathscr{X}}$ satisfies (AMC).

We now consider (ii) and (iii). Clearly, $(\mathrm{ACl}) \Rightarrow(\mathrm{AC})$. We show that $(\mathrm{AC}) \Rightarrow(\mathrm{AC} 1)$. To do so take any $a \in A$ and any $\mathscr{U} \in \operatorname{Cov}\left(X_{a}\right)$. Take a $\mathscr{V} \in \operatorname{Cov}\left(X_{a}\right)$ such that

$$
\text { st } \mathscr{V}<\mathscr{U} \text {. }
$$

By (A2) and (A3), there exists an $a_{1}>a$ such that

$$
\begin{gathered}
\left(p_{a a^{\prime}} p_{a^{\prime} a^{\prime \prime}}, p_{a a^{\prime \prime}}\right)<\mathscr{V} \text { for any } a^{\prime \prime}>a^{\prime}>a_{1}, \\
p_{a a^{\prime}}^{-1} \mathscr{V}>\text { st } \mathscr{U}_{a^{\prime \prime}} \text { for any } a^{\prime}>a_{1} .
\end{gathered}
$$

By the assumption, there exists an $a_{2}>a_{1}$ satisfying condition (AC) for $a_{1}$.

We show that $a_{2}$ is the required index. Take any $a^{\prime \prime}>a_{2}$ and any map $h: X_{a^{\prime \prime}} \rightarrow X_{a_{2}}$. By the choice of $a_{2}$ and (AC), there exist a $z \in X_{a^{\prime \prime}}$ and a $U_{1} \in$ st $\mathscr{U}_{a_{1}}$ such that

$$
p_{a_{1} a_{2}} h(z), p_{a_{1} a_{2}} p_{a_{2} a^{\prime \prime}}(z) \in U_{1} .
$$

By (14), there exists a $V_{1} \in \mathscr{V}$ such that $p_{a a_{1}}^{-1} V_{1} \supset U_{1}$. Then, by (15),

$$
p_{a a_{1}} p_{a_{1} a_{2}} h(z), p_{a a_{1}} p_{a_{1} a_{2}} p_{a_{2} a^{\prime \prime}}(z) \in V_{1} \text {. }
$$

By (13), $\left(p_{a a_{1}} p_{a_{1} a_{2}}, p_{a a_{2}}\right)<\mathscr{V}$. Thus, there exist $V_{2}, V_{3} \in \mathscr{V}$ such that

$$
\begin{gathered}
p_{a a_{1}} p_{a_{1} a_{2}} h(z), p_{a a_{2}} h(z) \in V_{2}, \\
p_{a a_{1}} p_{a_{1} a_{2}} p_{a_{2} a^{\prime \prime}}(z), p_{a a_{2}} p_{a_{2} a^{\prime \prime}}(z) \in V_{3} .
\end{gathered}
$$

By (16), (17), (18), and (12), there exists a $U \in \mathscr{U}$ such that

$$
p_{a a_{2}} h(z), p_{a a_{2}} p_{a_{2} a^{\prime \prime}}(z) \in V_{1} \cup V_{2} \cup V_{3} \subset \operatorname{st}\left(V_{1}, \mathscr{V}\right) \subset U .
$$

This means that $a_{2}$ is the required index. Hence $\mathscr{X}$ satisfies (AC1).

We say that an approximate resolution $\mathbf{p}: X \rightarrow \mathscr{X}$ satisfies $(\mathrm{AC})$ or is cosmic provided $\mathscr{X}$ satisfies (AC).

(5.2) Lemma. Let $\mathbf{p}: X \rightarrow \mathscr{X}$ and $\mathbf{q}: Y \rightarrow \mathscr{Y}$ be cofinite approximate APOL-resolutions. Let $X$ and $Y$ be homeomorphic. Then $\mathbf{p}$ satisfies (AC) if and only if $\mathbf{q}$ satisfies (AC).

Proof. Let $f: X \rightarrow Y$ be a homeomorphism. By (1.15) there exists an approximate resolution ( $\mathbf{p}, \mathbf{q}, \mathbf{f})$ of $f$. By Corollary (1.18), [f] is an isomorphism.

First, we assume that p satisfies (AC), i.e., $\mathscr{X}$ satisfies $(\mathrm{AC})$. Then by Lemma (5.1), [1 $\left.1_{\mathscr{Z}}\right]$ satisfies (AMC). By Lemma (4.2), [f][1 $\left.1_{\mathscr{Z}}\right]$ satisfies (AMC). Since $[\mathbf{f}]\left[1_{\mathscr{Z}}\right]=[\mathbf{f}]=\left[1_{\mathscr{y}}\right][\mathbf{f}],\left[1_{\mathscr{y}}\right][\mathbf{f}]$ satisfies $(\mathrm{AMC})$. Thus, by Lemma (4.4), [1 1$]$ 
satisfies (AMC). Hence, by Lemma (5.1), $\mathscr{Y}$ satisfies (AC), i.e., q satisfies (AC).

Applying the above fact to the homeomorphism $f^{-1}: Y \rightarrow X$, we have the converse assertion.

(5.3) Corollary. Let $\mathbf{p}: X \rightarrow \mathscr{X}$ and $\mathbf{p}^{\prime}: X \rightarrow \mathscr{X}^{\prime}$ be cofinite approximate APOL-resolutions. Then $\mathbf{p}$ satisfies (AC) if and only if $\mathbf{p}^{\prime}$ satisfies (AC).

By Corollary (5.3), we say that a space $X$ satisfies (AC) or is cosmic provided any/some cofinite approximate APOL-resolution $\mathbf{p}: X \rightarrow \mathscr{X}$ of $X$ satisfies $(\mathrm{AC})$.

By Lemma (5.2), we have that

(5.4) Corollary. Property (AC) is a topological invariant.

Lemmas (4.4) and (1.17) imply that

(5.5) Corollary. Let $f: X \rightarrow Y$ and $g: Y \rightarrow Z$ be maps. If $g f: X \rightarrow Z$ satisfies (AMC), then so does $g$.

(5.6) Corollary. If $f: X \rightarrow Y$ satisfies (AMC), then $Y$ satisfies (AC).

(5.7) Example. Holsztynski [H] only considers universality in terms of a fixed morphism between commutative inverse systems and the induced map on the limit. In our approach a given map may be induced by many different morphisms of approximate systems associated with the given map. Some of the expansions of a given map may be universal in the sense of Holsztynski and others may not. To see this consider a metric continuum $X$ which is the inverse limit of both a sequence of arcs and of a sequence of circles, i.e., $X$ is arc-like and circle-like. Any indecomposable arc-like continuum has this property (see [Bu, Theorem 7]). Then $X$ has an expansion $\mathbb{X}$ whose coordinate spaces are all arcs and an expansion of the identity map $1_{X}: X \rightarrow X, \mathbf{f}: \mathbb{X} \rightarrow \mathbb{X}$ given by $f_{i}=1_{X_{i}}$, which is universal in the sense of Holsztynski. On the other hand, $X$ also has an expansion $\mathbb{X}^{\prime}$ whose coordinate spaces are all circles and an expansion of $1_{X}, \mathbf{f}^{\prime}: \mathbb{X}^{\prime} \rightarrow \mathbb{X}^{\prime}$ given by $f_{i}^{\prime}=1_{X_{i}^{\prime}}$, which is not universal in the sense of Holsztynski. Therefore universality in the sense of Holsztynski depends on which expansion one uses and so it is not categorical.

We easily show

(5.8) Lemma. If $\mathscr{X}$ satisfies condition (US), then it satisfies (AC).

$$
\left(\forall a, \forall a^{\prime} \in A\right)\left(a^{\prime}>a\right)\left(\forall h: X_{a^{\prime}} \rightarrow X_{a}\right)\left(\exists x \in X_{a^{\prime}}\right) h(x)=p_{a a^{\prime}}(x) .
$$

(5.9) Remark. In (5.8), if $\mathscr{X}$ is commutative, then the condition (US) is equivalent to unversality in the sense of Holsztynski $[\mathrm{H}]$ and Mioduszewski and Rochowski [MiR]. Note that the condition (US) is not an approximate shape invariant, i.e., it depends on (approximate) polyhedral resolutions (see Example (5.7)).

Let $X$ be a compact Hausdorff space and $f, g: X \rightarrow Y$ be continuous maps. Let $\mathscr{U}$ be an open covering of $Y$. We say that a point $x \in X$ is a $\mathscr{U}$-coincidence point of $f$ and $g$ provided that $f(x), g(x) \in U$ for some $U \in \mathscr{U}$. 
(5.10) Lemma. Let $X$ be compact Hausdorff and $Y$ Tychonoff. If $f$ and $g$ have a $\mathscr{U}$-coincidence point for any $\mathscr{U} \in \operatorname{Cov}(Y)$, then $f$ and $g$ have a coincidence point. Moreover, if $g$ is the identity map, then $f$ has a fixed point.

Proof. Let $F(\mathscr{U})=\{x \in X ; x$ is a $\mathscr{U}$-coincidence point of $f$ and $g\}$ for any $\mathscr{U} \in \operatorname{Cov}(Y)$. By the definition, we have

Claim 1. If $\mathscr{V}<\mathscr{U}$, then $F(\mathscr{V}) \subset F(\mathscr{U})$.

Now, we show that

Claim 2. If st $\mathscr{V}<\mathscr{U}$, then $\overline{F(\mathscr{V})} \subset F(\mathscr{U})$.

To prove Claim 2, take any $x \in \overline{F(\mathscr{V})}$. There exist $V_{1}, V_{2} \in \mathscr{V}$ such that

$$
f(x) \in V_{1} \text { and } g(x) \in V_{2} \text {. }
$$

Since $f^{-1} V_{1} \cap g^{-1} V_{2}$ is an open neighborhood of $x$ by (1), $f^{-1} V_{1} \cap g^{-1} V_{2} \cap$ $F(\mathscr{V}) \neq \varnothing$. So there exists a point $y$ such that

$$
y \in f^{-1} V_{1} \cap g^{-1} V_{2} \cap F(\mathscr{V}) .
$$

By (2),

$$
f(y) \in V_{1} \text { and } g(y) \in V_{2} .
$$

Since $y \in F(\mathscr{V})$, there exists a $V_{3} \in \mathscr{V}$ such that

$$
f(y), g(y) \in V_{3} .
$$

By (1), (3), and (4), we have that $f(x), g(x) \in V_{1} \cup V_{2} \cup V_{3} \subset \operatorname{st}\left(V_{3}, \mathscr{V}\right)$. Since st $\mathscr{V}<\mathscr{U}$, there exists a $U \in \mathscr{U}$ such that $\operatorname{st}\left(V_{3}, \mathscr{V}\right) \subset U$. Then, $f(x), g(x) \in U$, i.e., $x$ is a $\mathscr{U}$-coincidence point of $f$ and $g$. Therefore we have Claim 2.

Let $j_{\mathscr{U}, \mathscr{V}}: \overline{F(\mathscr{V})} \rightarrow \overline{F(\mathscr{U})}$ be the inclusion map for each $\mathscr{V}, \mathscr{U} \in \operatorname{Cov}(Y)$ with $\mathscr{V}<\mathscr{U}$. Thus $\mathbb{F}(f, g)=\left\{\overline{\boldsymbol{F}(\mathscr{U})}, j_{\mathscr{U}, \mathscr{V}}, \operatorname{Cov}(Y)\right\}$ forms an inverse system. Let $F(f, g)$ be the set of all coincidence points of $f$ and $g$.

Claim 3. $F(f, g)=\lim \mathbb{F}(f, g)$.

Clearly $\lim \mathbb{F}(f, g)=\bigcap\{\overline{F(\mathscr{U})}: \mathscr{U} \in \operatorname{Cov}(Y)\}$. Since $F(f, g) \subset F(\mathscr{U}) \subset$ $\overline{F(\mathscr{U})}$ for any $\mathscr{U} \in \operatorname{Cov}(Y), F(f, g) \subset \bigcap\{\overline{F(\mathscr{U})}: \mathscr{U} \in \operatorname{Cov}(Y)\}$.

We must show that $\bigcap\{\overline{F(\mathscr{U})}: \mathscr{U} \in \operatorname{Cov}(Y)\} \subset F(f, g)$. Take any $x \in$ $\bigcap\{\overline{F(\mathscr{U})}: \mathscr{U} \in \operatorname{Cov}(Y)\}$. We assume that $x \notin F(f, g)$, i.e., $f(x) \neq g(x)$. Since $Y$ is Tychonoff, there exists a $\mathscr{U} \in \operatorname{Cov}(Y)$ such that

$$
\operatorname{st}(f(x), \mathscr{U}) \cap \operatorname{st}(g(x), \mathscr{U})=\varnothing .
$$

There exists $\mathscr{V} \in \operatorname{Cov}(Y)$ such that st $\mathscr{V}<\mathscr{U}$. By Claim 2 and the choice of $x$,

$$
x \in \overline{F(\mathscr{V})} \subset F(\mathscr{U}) .
$$

By (6), $x \in F(\mathscr{U})$, i.e., there exists a member $K \in \mathscr{U}$ such that $f(x), g(x) \in$ $K$. By (5), such a $K$ does not exist. Thus, we have a contradiction. Thus, $f(x)=g(x)$, i.e., $x \in F(f, g)$. Therefore, $\bigcap\{\overline{F(\mathscr{U})}: \mathscr{U} \in \operatorname{Cov}(Y)\} \subset$ $F(f, g)$ and we have Claim 3.

Since $\mathbb{F}(f, g)$ is an inverse system consisting of nonempty compact Hausdorff spaces $\overline{F(\mathscr{U})}, \lim \mathbb{F}(f, g)$ is not empty. By Claim $3, F(f, g)$ is not empty. Hence, $f$ and $g$ have a coincidence point and the proof is complete. 
(5.11) Theorem. Let $f, g: X \rightarrow Y$ be maps from a compact Hausdorff space $X$ to a Tychonoff space $Y$. Then their approximate APOL-resolutions satisfy (AN) if and only if $f$ and $g$ have a coincidence point.

Theorem (5.11) follows from Lemmas (3.1) and (5.10).

(5.12) Lemma. If $X$ satisfies (AC), then any map $f: X \rightarrow X$ has a $\mathscr{V}$-fixed point for any $\mathscr{V} \in \operatorname{Cov}(X)$.

Proof. Let $\mathbf{p}: X \rightarrow \mathscr{Z}$ be a cofinite approximate APOL-resolution. By the assumption, $\mathscr{X}$ satisfies (AC). By Lemma (5.1), [1 $\mathscr{X}]: \mathscr{X} \rightarrow \mathscr{X}$ satisfies (AMC). Since $1_{\mathscr{X}}: \mathscr{X} \rightarrow \mathscr{X}$ is an approximate resolution of $1_{X}: X \rightarrow X$, then $1_{X}$ satisfies the condition (i) in Lemma (4.8). Hence, we have the required property.

(5.13) Theorem. If $X$ is a compact Hausdorff space with a cosmic approximate ANR-resolution, then $X$ has the fixed point property.

Theorem (5.13) follows from Lemmas (5.10) and (5.12).

(5.14) Corollary. Let $\mathbf{p}: X \rightarrow(\mathscr{X}, \mathscr{U})$ be an approximate resolution of compact Hausdorff spaces $X_{a}$. If $\mathscr{Z}$ satisfies (US), then $X$ has the fixed point property.

(5.15) Corollary. If $X$ is an arc-like Hausdorff continuum, then $X$ has the fixed point property.

Proof. By [MM] $X$ has an approximate resolution $\mathbf{p}=\left\{p_{a}: a \in A\right\}: X \rightarrow$ $\mathscr{X}=\left\{X_{a}, \mathscr{U}_{a}, p_{a a^{\prime}}, A\right\}$ such that each $X_{a}$ is an arc and each $p_{a a^{\prime}}$ is surjective. Since $p_{a a^{\prime}}$ is surjective, $p_{a a^{\prime}}$ is universal by Proposition 8 of $[\mathrm{H}]$ and so $\mathscr{X}$ satisfies property (US). Hence, by (5.14), $X$ has the fixed point property.

(5.16) Remark. This corollary extends a similar result in $[\mathrm{H}]$ to the Hausdorff case. Mardešić $[\mathrm{M}]$ constructed an arc-like continuum which is not an inverse limit of any (commutative) inverse system of arcs. Therefore, we need the notion of approximate resolution for our proof. The approximate limit approach also allows for a generalization of fixed point results for tree-like continua to the nonmetric case. Marsh [Ma] has introduced a class of maps between trees, called $u$-maps, which are cosmic. So if a tree-like Hausdorff continuum $X$ has an approximate resolution as trees and $u$-mappings as bonding maps, then $X$ has the fixed point property.

(5.17) Corollary. If $X$ is an essentially ( $n$-cell)-like Hausdorff continuum, $n \geq$ 2 , then $X$ has the fixed point property.

Proof. Let $B^{n}$ be an $n$-cell and $S^{n-1}$ be its boundary. The hypothesis means $X$ has an approximate resolution with $n$-cells $B^{n}$ as coordinate spaces $X_{a}$ and bonding maps $p_{a a^{\prime}}: X_{a^{\prime}} \rightarrow X_{a}$ such that $p_{a a^{\prime}} \mid p_{a a^{\prime}}^{-1}\left(S^{n-1}\right): p_{a a^{\prime}}^{-1}\left(S^{n-1}\right) \rightarrow$ $S^{n-1}$ is essential (i.e., cannot be extended to a map of $B^{n}$ to $S^{n-1}$ ). Then by Proposition 10 of $[\mathrm{H}], \mathscr{X}$ satisfies (US). Therefore $X$ has the fixed point property by (5.14).

(5.18) Remark. Note that essentiality is necessary since there are (3-cell)-like continua without the fixed point property (see [Bol] or [B, Theorem 20]). 
We now give an example of continuum having the fixed point property but which is not cosmic.

(5.19) Example. The Warsaw Circle is a continuum $X$ which has the fixed point property, but does not satisfy property (AC).

We consider points $a_{n}, b_{n}, n=0,1,2, \ldots, c_{0}$ and $c_{1}$ in the plane as follows: $a_{0}=(0,-1), a_{n}=\left(\frac{1}{n},-1\right)$ for $n=1,2, \ldots, c_{0}=(0,0), c_{1}=$ $(1,0), b_{0}=(0,1), b_{n}=\left(\frac{1}{n}, 1\right)$ for each odd $n$, and $b_{n}=\left(\frac{1}{n}, 0\right)$ for each even $n$. Then $X=\overline{b_{0} a_{0}} \cup \overline{a_{0} a_{1}} \cup \overline{a_{1} b_{1}} \cup \bigcup_{i=1}^{\infty} \overline{b_{i} b_{i+1}}$ is a continuum in the plane $R^{2}$.

Let

$$
X_{n}=\overline{b_{0} c_{0}} \cup \overline{c_{0} a_{0}} \cup \overline{a_{0} a_{1}} \cup \overline{a_{1} c_{1}} \cup \overline{c_{1} b_{1}} \cup \bigcup_{i=1}^{2 n-1} \overline{b_{i} b_{i+1}} \cup \overline{b_{2 n} b_{0}}
$$

be a simplicial complex in $R^{2}$ for $n \geq 1$. Let $p_{n m}: X_{m} \rightarrow X_{n}$ be a simplicial map defined for $m \geq n$ by

$$
p_{n m}(v)= \begin{cases}b_{0} & \text { if } v=b_{j}, j \text { is odd and } 2 m \geq j>2 n \\ c_{0} & \text { if } v=b_{j}, j \text { is even and } 2 m \geq j>2 n \\ v & \text { otherwise }\end{cases}
$$

It is easy to show that $p_{m_{1} m_{2}} p_{m_{2} m_{3}}=p_{m_{1} m_{3}}$ for $m_{1} \leq m_{2} \leq m_{3}$. Then $\mathbb{X}=$ $\left\{X_{n}, p_{n m}, N^{+}\right\}$is an (commutative) inverse sequence. Here $N^{+}$is the set of all positive integers with the usual order.

Let $\varphi_{n}:\left\{b_{0}, c_{0}, a_{0}, a_{1}, c_{1}\right\} \cup\left\{b_{i}: i=1,2, \ldots\right\} \rightarrow X_{n}^{0}=\left\{b_{0}, c_{0}, a_{0}, a_{1}, c_{1}\right\}$ $\cup\left\{b_{i}: i=1,2, \ldots, 2 n\right\}$ be a map defined by

$$
\varphi_{n}(v)= \begin{cases}b_{0} & \text { if } v=b_{j}, j \text { is odd and } j>2 n \\ c_{0} & \text { if } v=b_{j}, j \text { is even and } j>2 n \\ v & \text { otherwise }\end{cases}
$$

Clearly $\varphi_{n}$ defines a map $p_{n}: X \rightarrow X_{n}$. Since $\varphi_{n}=p_{n m} \varphi_{m}$ for $n \leq m$, we have $p_{n}=p_{n m} p_{m}$. Thus $\mathbf{p}=\left\{p_{n}: n \in N^{+}\right\}: X \rightarrow \mathbb{X}$ is a (commutative) system map. It is not difficult to show that $\mathbf{p}: X \rightarrow \mathbb{X}$ is an inverse limit. By Theorem 8 of [M1], $\mathbf{p}$ is a resolution. Then by (3.9) of [W1], there are coverings $\mathscr{U}_{i} \in \operatorname{Cov}\left(X_{i}\right)$ such that $\mathbf{p}: X \rightarrow(\mathscr{X}, \mathscr{U})=\left\{X_{n}, \mathscr{U}_{n}, p_{n m}, N^{+}\right\}$is an (commutative) approximate resolution of $X$.

We show that $(\mathscr{X}, \mathscr{U})$ does not satisfy property $(\mathrm{AC})$. We assume that $(\mathscr{X}, \mathscr{U})$ satisfies condition (AC). By Lemma (5.1), it satisfies condition (AC1). Take any $n \in N^{+}$and any covering $\mathscr{V}_{n}$ of $X_{n}$ such that the mesh of $\mathscr{V}_{n}<1$. Then by $(\mathrm{ACl} 1)$, there exists an $n_{0} \geq n$ satisfying

(1) for each $m \geq n_{0}$ and each map $h: X_{m} \rightarrow X_{n_{0}}$, there exists a point $x \in X_{m}$ and a $U \in \mathscr{V}_{n}$ such that $p_{n m}(x), p_{n n_{0}} h(x) \in U$.

Take any $m \geq n_{0}$. We define a map $h: X_{m} \rightarrow X_{m}$ as follows: We subdivide $X_{m}$ by

$$
\overline{b_{0} c_{0}} \cup \overline{c_{0} a_{0}} \cup \overline{a_{0} a_{2 m}} \cup \bigcup_{j=1}^{2 m-1} \overline{a_{j+1} a_{j}} \cup \overline{a_{1} c_{1}} \cup \overline{c_{1} b_{1}} \cup \bigcup_{j=1}^{2 m-1} \overline{b_{j} b_{j+1}} \cup \overline{b_{2 m} b_{0}}
$$


Let $h: X_{m} \rightarrow X_{m}$ be a simplicial map defined by

$$
h(v)= \begin{cases}a_{1} & \text { if } v=b_{0}, \\ c_{1} & \text { if } v=c_{0}, \\ b_{1} & \text { if } v=a_{0}, \\ b_{j+2} & \text { if } v=a_{2 m-j}, 0 \leq j \leq 2 m-2, \\ b_{0} & \text { if } v=a_{1}, \\ c_{0} & \text { if } v=c_{1}, \\ a_{0} & \text { if } v=b_{1}, \\ a_{2 m+2-j} & \text { if } v=b_{j}, 2 \leq j \leq 2 m .\end{cases}
$$

It is easy to show that

(2) if $x \in \overline{b_{0} c_{0}} \cup \overline{c_{0} a_{0}} \cup \overline{a_{1} c_{1}} \cup \overline{c_{1} b_{1}}=K$, then $\left|\pi_{1} p_{n m}(x)-\pi_{1} p_{n m} h(x)\right|=1$,

(3) if $x \in X_{m}-K$, then $\left|\pi_{2} p_{n m}(x)-\pi_{2} p_{n m} h(x)\right| \geq 1$.

Here $\pi_{i}: R^{2} \rightarrow R$ is the projection to the $i$ th coordinate for each $i=1,2$. By (2) and (3), for each $x \in X_{m}$, the distance between $p_{n m}(x)$ and $p_{n m} h(x)$ is not less than 1 . Since the mesh of $\mathscr{V}_{n}<1$, we cannot find a member $U \in \mathscr{V}_{n}$ containing both points $p_{n m}(x)$ and $p_{n m} h(x)$ for each $x \in X_{m}$. Thus, this contradicts $(1)$. Hence $(\mathscr{X}, \mathscr{U})$ does not satisfy property (AC). However, it is well known that $X$ has the fixed point property (see Theorem 13 of [B]).

\section{COMPLEX PROJECTIVE $n$-SPACE LIKE CONTINUA AND THE FIXED POINT PROPERTY}

Let $P^{n}(\mathbb{C})$ be the $n$-dimensional complex projective space. Note that the geometrical dimension of $P^{n}(\mathbb{C})$ is $2 n$. The following data is well known:

$$
\begin{aligned}
H_{j}\left(P^{n}(\mathbb{C}) ; \mathbb{Z}\right) & = \begin{cases}\mathbb{Z} & \text { for } j=0,2,4, \ldots, 2 n, \\
0 & \text { otherwise },\end{cases} \\
\pi_{j}\left(P^{n}(\mathbb{C})\right) & = \begin{cases}0, & j \leq 2 n \text { and } j \neq 2, \\
\mathbb{Z}, & j=2 .\end{cases}
\end{aligned}
$$

Here, $\mathbb{Z}$ is the additive group of all integers, and $\mathbb{C}$ is the field of all complex numbers. Let $\mathbb{Q}$ be the field of all rational numbers.

$$
\begin{aligned}
& P^{n}(\mathbb{C}) \text { is a } 2 n \text {-dimensional orientable manifold without bound- } \\
& \text { ary. Moreover, it is simply connected. }
\end{aligned}
$$

Let $f: P^{n}(\mathbb{C}) \rightarrow P^{n}(\mathbb{C})$ be a map. Then, $f$ induces a homomorphism $f_{*}: H_{2}\left(P^{n}(\mathbb{C}) ; \mathbb{Z}\right) \rightarrow H_{2}\left(P^{n}(\mathbb{C}) ; \mathbb{Z}\right)$. Since $H_{2}\left(P^{n}(\mathbb{C}) ; \mathbb{Z}\right) \cong \mathbb{Z}$ by $(1)$, we take a generator $\alpha$ of $H_{2}\left(P^{n}(\mathbb{C}) ; \mathbb{Z}\right)$ and then $H_{2}\left(P^{n}(\mathbb{C}) ; \mathbb{Z}\right)=\{k \alpha: k \in \mathbb{Z}\}$. We may define a $\lambda \in \mathbb{Z}$ by $f_{*}(\alpha)=\lambda \alpha$. We say that $\lambda$ is the 2-dimensional degree of $f$ and write it as $\lambda=\operatorname{deg}(f)$. Note that $\lambda$ does not depend on the choice of the generator $\alpha$. (See the proof of (7).)

By the universal coefficient theorem, we have the following exact sequence:

$$
\begin{aligned}
0 & \rightarrow \operatorname{Ext}\left(H_{j-1}\left(P^{n}(\mathbb{C}) ; \mathbb{Z}\right), \mathbb{Q}\right) \rightarrow H^{j}\left(P^{n}(\mathbb{C}) ; \mathbb{Q}\right) \\
& \rightarrow \operatorname{Hom}\left(H_{j}\left(P^{n}(\mathbb{C}) ; \mathbb{Z}\right), \mathbb{Q}\right) \rightarrow 0 .
\end{aligned}
$$

By (1) and (4), we have

$$
H^{j}\left(P^{n}(\mathbb{C}) ; \mathbb{Q}\right) \cong \begin{cases}\mathbb{Q} & \text { for } j=0,2,4, \ldots, 2 n, \\ 0 & \text { otherwise. }\end{cases}
$$


By the naturality of the universal coefficient theorem,

$$
\begin{aligned}
& H^{2}\left(P^{n}(\mathbb{C}) ; \mathbb{Q}\right) \cong \operatorname{Hom}\left(H_{2}\left(P^{n}(\mathbb{C}) ; \mathbb{Z}\right), \mathbb{Q}\right) \cong \operatorname{Hom}(\mathbb{Z}, \mathbb{Q}) \cong \mathbb{Q} \\
& \quad f^{*} \downarrow \quad \operatorname{Hom}\left(f_{*}, 1_{\mathbb{Q}}\right) \downarrow \\
& H^{2}\left(P^{n}(\mathbb{C}) ; \mathbb{Q}\right) \cong \operatorname{Hom}\left(H_{2}\left(P^{n}(\mathbb{C}) ; \mathbb{Z}\right), \mathbb{Q}\right) \cong \operatorname{Hom}(\mathbb{Z}, \mathbb{Q}) \cong \mathbb{Q},
\end{aligned}
$$

where $\lambda=\operatorname{deg}(f)$ and $\times \lambda$ is the homomorphism determined by multiplication by $\lambda$. Let $\beta$ be the generator of $H^{2}\left(P^{n}(\mathbb{C}) ; \mathbb{Q}\right)$ determined by $\varphi^{-1}(1)$, where $\varphi$ is an isomorphism in $(5)$ and $1 \in \mathbb{Q}$. Thus $H^{2}\left(P^{n}(\mathbb{C}) ; \mathbb{Q}\right)=\{r \beta: r \in \mathbb{Q}\}$ and $f^{*}(r \beta)=\lambda r \beta$ for each $r \in \mathbb{Q}$.

Let $\beta^{\prime}$ be another generator of $H^{2}\left(P^{n}(\mathbb{C}) ; \mathbb{Q}\right)$. We put $f^{*}\left(\beta^{\prime}\right)=\lambda^{\prime} \beta^{\prime}, \lambda^{\prime} \in$ $\mathbb{Q}$. We show that

$$
\lambda=\lambda^{\prime} .
$$

Since $\beta, \beta^{\prime}$ are generators, $\beta \neq 0$ and $\beta^{\prime} \neq 0$. We put $\beta=t \beta^{\prime}$, where $t=\frac{t_{1}}{t_{2}} \in \mathbb{Q}$ and $t_{1}, t_{2} \in \mathbb{Z}$. Since $\beta \neq 0$ and $\beta^{\prime} \neq 0$, then $t_{1} \neq 0$ and $t_{2} \neq 0$. Since $t_{2} \beta=t_{1} \beta^{\prime}, t_{2} \lambda \beta=t_{2} f_{*}(\beta)=f_{*}\left(t_{2} \beta\right)=f_{*}\left(t_{1} \beta^{\prime}\right)=t_{1} f_{*}\left(\beta^{\prime}\right)=t_{1} \lambda^{\prime} \beta^{\prime}$, i.e., $t_{1} \lambda^{\prime} \beta^{\prime}=t_{2} \lambda \beta$. Since $\beta=\frac{t_{1}}{t_{2}} \beta^{\prime}, t_{1} \lambda^{\prime} \beta^{\prime}=t_{2} \lambda \beta=t_{2} \lambda t_{t_{2}} \beta^{\prime}=\lambda t_{1} \beta^{\prime}$. Since $\beta^{\prime} \neq 0$ and $t_{1} \neq 0, t_{1} \lambda^{\prime} \beta^{\prime}=\lambda t_{1} \beta^{\prime}$ implies $\lambda^{\prime}=\lambda$ and we have (7). Thus we have that

$$
\begin{aligned}
& f^{*}: H^{2}\left(P^{n}(\mathbb{C}) ; \mathbb{Q}\right) \rightarrow H^{2}\left(P^{n}(\mathbb{C}) ; \mathbb{Q}\right) \text { satisfies } f^{*}(\beta)=\lambda \beta \text { for } \\
& \text { any generator } \beta \text { of } H^{2}\left(P^{n}(\mathbb{C}) ; \mathbb{Q}\right) .
\end{aligned}
$$

Hence $\lambda=\operatorname{deg}(f)$.

Now, $\mathbb{Q}$ is a commutative field. It is well known that $H^{*}\left(P^{n}(\mathbb{C}) ; \mathbb{Q}\right)$ forms a cohomological ring such that $H^{*}\left(P^{n}(\mathbb{C}) ; \mathbb{Q}\right)$ is a truncated polynomial ring over $\mathbb{Q}$ generated by a generator $x$ of $H^{2}\left(P^{n}(\mathbb{C}) ; \mathbb{Q}\right)$ and height $n+1$, i.e.,

$$
H^{*}\left(P^{n}(\mathbb{C}) ; \mathbb{Q}\right) \cong P_{\mathbb{Q}}[x] /\left(x^{n+1}\right) .
$$

Here $P_{\mathbb{Q}}[x]$ is the ring of polynomials generated by $x$ with rational coefficients, and $\left(x^{n+1}\right)$ is an ideal generated by $x^{n+1}$. Note that the cup product induces the ring product structure of $H^{*}\left(P^{n}(\mathbb{C}) ; \mathbb{Q}\right)$. (9) means that

$$
x^{j} \text { is a generator of } H^{2 j}\left(P^{n}(\mathbb{C}) ; \mathbb{Q}\right) .
$$

By (8), we have that

$$
f^{*}(x)=\lambda x, \quad \text { where } f^{*}: H^{2}\left(P^{n}(\mathbb{C}) ; \mathbb{Q}\right) \rightarrow H^{2}\left(P^{n}(\mathbb{C}) ; \mathbb{Q}\right) .
$$

By $(10)$ and $(11), f^{*}: H^{2 j}\left(P^{n}(\mathbb{C}) ; \mathbb{Q}\right) \rightarrow H^{2 j}\left(P^{n}(\mathbb{C}) ; \mathbb{Q}\right)$ has the property:

$$
f^{*}\left(x^{j}\right)=f^{*} \overbrace{(x \cdots x)}^{j \text { times }}=\overbrace{f^{*}(x) \cdots f^{*}(x)}^{j \text { times }}=(\lambda x) \cdots(\lambda x)=\lambda^{j} x^{j} .
$$

Hence

$$
f^{*}\left(x^{j}\right)=\lambda^{j} x^{j} \quad \text { for } j=1,2, \ldots, n .
$$

Take any $j=0,1,2, \ldots, n$. Since $f$ induces a homomorphism $f_{*}$ : $H_{2 j}\left(P^{n}(\mathbb{C}) ; \mathbb{Z}\right) \rightarrow H_{2 j}\left(P^{n}(\mathbb{C}) ; \mathbb{Z}\right)$, by $(1)$ there exists a $\tilde{\lambda}_{2 j} \in \mathbb{Z}$ such that $f_{*}\left(\alpha_{2 j}\right)=\tilde{\lambda}_{2 j} \alpha_{2 j}$, where $\alpha_{2 j} \in H_{2 j}\left(P^{n}(\mathbb{C}) ; \mathbb{Z}\right)$ is a generator. Note that $\tilde{\lambda}_{2 j}$ does not depend on the choice of the generator $\alpha_{2 j}$. Using the same argument as $(6)-(8)$ we have by $(5)$ that for $f^{*}: H^{2 j}\left(P^{n}(\mathbb{C}) ; \mathbb{Q}\right) \rightarrow H^{2 j}\left(P^{n}(\mathbb{C}) ; \mathbb{Q}\right)$, 
$f^{*}\left(\beta_{2 j}\right)=\tilde{\lambda}_{2 j} \beta_{2 j}$ for any generator $\beta_{2 j}$ of $H^{2 j}\left(P^{n}(\mathbb{C}) ; \mathbb{Q}\right)$ for $j=0,1, \ldots, n$. However, by (10) and (12), we have $\tilde{\lambda}_{2 j}=\lambda^{j}$. Hence, for $f_{*}: H_{2 j}\left(P^{n}(\mathbb{C}) ; \mathbb{Z}\right) \rightarrow$ $H_{2 j}\left(P^{n}(\mathbb{C}) ; \mathbb{Z}\right)$,

$$
f_{*}\left(\beta_{2 j}\right)=\lambda^{j} \beta_{2 j}
$$

for any generator $\beta_{2 j} \in H_{2 j}\left(P^{n}(\mathbb{C}) ; \mathbb{Z}\right)$ and $j=0,1,2, \ldots, n$.

By the universal coefficient theorem, we have

(14) $0 \rightarrow H_{j}\left(P^{n}(\mathbb{C}) ; \mathbb{Z}\right) \otimes \mathbb{Q} \stackrel{\psi}{\rightarrow} H_{j}\left(P^{n}(\mathbb{C}) ; \mathbb{Q}\right) \rightarrow \operatorname{Tor}\left(H_{j-1}\left(P^{n}(\mathbb{C}) ; \mathbb{Z}\right), \mathbb{Q}\right) \rightarrow 0$

for each $j$. Thus, by (1),

$$
H_{j}\left(P^{n}(\mathbb{C}) ; \mathbb{Q}\right) \cong \begin{cases}H_{j}\left(P^{n}(\mathbb{C}) ; \mathbb{Z}\right) \otimes \mathbb{Q} \cong \mathbb{Z} \otimes \mathbb{Q} \cong \mathbb{Q} & \text { for } j=0,2, \ldots, 2 n, \\ 0 & \text { otherwise. }\end{cases}
$$

By (14) for $j=0,1,2, \ldots, n$,

$$
\begin{array}{cc}
H_{2 j}\left(P^{n}(\mathbb{C}) ; \mathbb{Z}\right) \otimes \mathbb{Q} \underset{\psi}{\lfloor} H_{2 j}\left(P^{n}(\mathbb{C}) ; \mathbb{Q}\right) \\
\downarrow_{*} \otimes 1_{\mathbb{Q}} & \downarrow_{*} \\
H_{2 j}\left(P^{n}(\mathbb{C}) ; \mathbb{Z}\right) \otimes \mathbb{Q} \underset{\psi}{\longrightarrow} H_{2 j}\left(P^{n}(\mathbb{C}) ; \mathbb{Q}\right)
\end{array}
$$

is commutative. Since $\psi$ is an isomorphism, by (13) and (16)

$$
f_{*}\left(\widetilde{\beta}_{2 j}\right)=\lambda^{j} \widetilde{\beta}_{2 j},
$$

where $\widetilde{\beta}_{2 j}=\psi\left(\beta_{2 j} \otimes 1\right)$.

By the same way as in (7), we can show that $\lambda^{j}$ does not depend on the choice of the generator $\beta_{2 j}$, i.e., for $f_{*}: H_{2 j}\left(P^{n}(\mathbb{C}) ; \mathbb{Q}\right) \rightarrow H_{2 j}\left(P^{n}(\mathbb{C}) ; \mathbb{Q}\right)$,

$$
f_{*}\left(\beta_{2 j}\right)=\lambda^{j} \beta_{2 j}
$$

for any generator $\beta_{2 j} \in H_{2 j}\left(P^{n}(\mathbb{C}) ; \mathbb{Q}\right)$ and $j=0,1, \ldots, n$.

Now, by (3) we have the Poincaré duality theorem: There exists an isomorphism

$$
D_{j}: H^{j}\left(P^{n}(\mathbb{C}) ; \mathbb{Q}\right) \rightarrow H_{2 n-j}\left(P^{n}(\mathbb{C}) ; \mathbb{Q}\right)
$$

for any $j=0,1,2, \ldots, 2 n$.

We define a Gysin homomorphism $f !: H^{j}\left(P^{n}(\mathbb{C}) ; \mathbb{Q}\right) \rightarrow H^{j}\left(P^{n}(\mathbb{C}) ; \mathbb{Q}\right)$ such that $f !=D_{j}^{-1} f_{*} D_{j}$, i.e.,

$$
\begin{array}{ccc}
H^{j}\left(P^{n}(\mathbb{C}) ; \mathbb{Q}\right) & \underset{f !}{\longrightarrow} & H^{j}\left(P^{n}(\mathbb{C}) ; \mathbb{Q}\right) \\
\cong \downarrow D_{j} & & \cong D_{j} \\
H_{2 n-j}\left(P^{n}(\mathbb{C}) ; \mathbb{Q}\right) & \longrightarrow f_{*} & H_{2 n-j}\left(P^{n}(\mathbb{C}) ; \mathbb{Q}\right)
\end{array}
$$

is commutative.

We show that for $f !: H^{2 j}\left(P^{n}(\mathbb{C}) ; \mathbb{Q}\right) \rightarrow H^{2 j}\left(P^{n}(\mathbb{C}) ; \mathbb{Q}\right)$,

$$
f !\left(x^{j}\right)=\lambda^{n-j} x^{j} \quad \text { for } j=0,1, \ldots, n .
$$


By $(10)$ and (19), $D_{2 j}\left(x^{j}\right)$ is a generator of $H_{2 n-2 j}\left(P^{n}(\mathbb{C}) ; \mathbb{Q}\right)$. By (18),

$$
f_{*}\left(D_{2 j}\left(x^{j}\right)\right)=\lambda^{n-j} D_{2 j}\left(x^{j}\right) \text {. }
$$

Thus,

$$
\begin{aligned}
f !\left(x^{j}\right) & =D_{2 j}^{-1} f_{*} D_{2 j}\left(x^{j}\right)=D_{2 j}^{-1}\left(\lambda^{n-j} D_{2 j}\left(x^{j}\right)\right) \\
& =\lambda^{n-j} D_{2 j}^{-1} D_{2 j}\left(x^{j}\right)=\lambda^{n-j} x^{j} .
\end{aligned}
$$

Thus we have (21).

We calculate the Lefschetz number $\lambda(f, g)$ for maps $f, g: P^{n}(\mathbb{C}) \rightarrow P^{n}(\mathbb{C})$ as follows.

(6.1) Lemma. Let $f, g: P^{n}(\mathbb{C}) \rightarrow P^{n}(\mathbb{C})$ be maps. Then $\lambda(f, g)=\lambda^{n}+$ $\lambda^{n-1} \mu+\lambda^{n-2} \mu^{2}+\cdots+\lambda \mu^{n-1}+\mu^{n}$. Here $\lambda=\operatorname{deg}(f)$ and $\mu=\operatorname{deg}(g)$.

Proof. Note that $\lambda(f, g)$ is defined by

$$
\lambda(f, g)=\sum_{j=0}^{\infty}(-1)^{j} \operatorname{trace}\left(g !^{j} f^{* j}\right)
$$

Here

$$
H^{j}\left(P^{n}(\mathbb{C}) ; \mathbb{Q}\right) \stackrel{f^{* j}}{\longrightarrow} H^{j}\left(P^{n}(\mathbb{C}) ; \mathbb{Q}\right) \stackrel{g !^{j}}{\longrightarrow} H^{j}\left(P^{n}(\mathbb{C}) ; \mathbb{Q}\right)
$$

Take any $j=0,1, \ldots, n$. By (12)

$$
f^{* 2 j}\left(x^{j}\right)=f^{*}\left(x^{j}\right)=\lambda^{j} x^{j} .
$$

By $(21)$, since $\mu=\operatorname{deg}(g)$,

$$
g !^{2 j}\left(x^{j}\right)=\mu^{n-j} x^{j}
$$

By (23) and (24)

$$
g !^{2 j} f^{* 2 j}\left(x^{j}\right)=\lambda^{j} \mu^{n-j} x^{j}
$$

Since $H^{2 j}\left(P^{n}(\mathbb{C}) ; \mathbb{Q}\right) \cong \mathbb{Q}$,

$$
\operatorname{trace}\left(g !^{2 j} f^{* 2 j}\right)=\lambda^{j} \mu^{n-j} \quad \text { for } j=0,1, \ldots, n \text {. }
$$

Let $j$ be an odd integer. By (5)

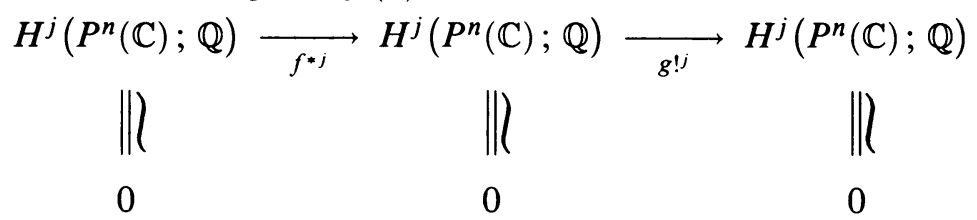

must be the zero homomorphism. Thus

$$
\operatorname{trace}\left(g !^{j} f^{* j}\right)=0 \quad \text { for any odd integer } j \text {. }
$$

Hence, by (26) and (27),

$$
\begin{aligned}
\lambda(f, g) & =\sum_{j=0}^{2 n}(-1)^{j} \operatorname{trace}\left(g !^{j} f^{* j}\right) \\
& =\sum_{j: \text { odd }}(-1)^{j} \operatorname{trace}\left(g !^{j} f^{* j}\right)+\sum_{j=0}^{n}(-1)^{2 j} \operatorname{trace}\left(g !^{2 j} f^{* 2 j}\right) \\
& =\sum_{j=0}^{n} \lambda^{j} \mu^{n-j} .
\end{aligned}
$$

Therefore, we have Lemma (6.1). 
(6.2) Lemma. Under the conditions of Lemma 6.1, we have

(i) Let $n$ be an even integer. Then $\lambda(f, g)=0$ if and only if $\operatorname{deg}(f)=$ $\operatorname{deg}(g)=0$.

(ii) Let $n$ be an odd integer. Then $\lambda(f, g)=0$ if and only if $\operatorname{deg}(f)=$ $-\operatorname{deg}(g)$.

Proof. Let $\operatorname{deg}(f)=\operatorname{deg}(g)=0$. Thus $\lambda=\mu=0$. By Lemma (6.1), $\lambda(f, g)=0$. Next, we assume that $\lambda(f, g)=0$. We show that

$$
\text { if } \lambda=0 \text {, then } \mu=0 \text {. }
$$

Since $0=\lambda(f, g)=\lambda^{n}+\lambda^{n-1} \mu+\cdots+\lambda \mu^{n-1}+\mu^{n}=\mu^{n}, \mu=0$. Similarly, we have

$$
\text { if } \mu=0 \text {, then } \lambda=0 \text {. }
$$

We show that

$$
\text { if } \lambda \neq 0, \text { then } \lambda \neq \mu \text {. }
$$

We assume that $\lambda \neq 0$ and $\lambda=\mu$. Thus,

$$
0=\lambda(f, g)=\lambda^{n}+\lambda^{n-1} \mu+\cdots+\lambda \mu^{n-1}+\mu^{n}=(n+1) \lambda^{n} .
$$

Thus $\lambda^{n}=0$ and hence $\lambda=0$. This contradicts $\lambda \neq 0$. Therefore $\lambda \neq \mu$.

We show that

$$
\text { if } \lambda \neq 0 \text {, then } \frac{\mu}{\lambda} \neq 0,1 \text {. }
$$

If $\frac{\mu}{\lambda}=0$, then $\mu=0$. By (2), $\lambda=0$. This contradicts the assumption $\lambda \neq 0$. Therefore $\frac{\mu}{\lambda} \neq 0$. Next, we put $\frac{\mu}{\lambda}=1$. Since $\frac{\mu}{\lambda}=1$, then $\lambda=\mu$. This contradicts (3). Hence $\frac{\mu}{\lambda} \neq 0,1$.

We assume that $\lambda \neq 0$. By (4), $y=\frac{\mu}{\lambda} \neq 0,1$. Since $\lambda(f, g)=0,0=$ $\lambda(f, g)=\lambda^{n}\left(1+\frac{\mu}{\lambda}+\left(\frac{\mu}{\lambda}\right)^{2}+\cdots+\left(\frac{\mu}{\lambda}\right)^{n}\right)$ and then

$$
1+y+\cdots+y^{n}=0 \text {. }
$$

Since $1+y+\cdots+y^{n}=\frac{1-y^{n+1}}{1-y}$, by $(5)$

$$
y^{n+1}=1 .
$$

Let $w=\cos \frac{2 \pi}{n+1}+i \sin \frac{2 \pi}{n+1}$. It is well known that the solutions of (6) are $y=1, w, w^{2}, \ldots, w^{n}$. Since $y \neq 1$, the solutions of $(5)$ are

$$
y=w, w^{2}, \ldots, w^{n}
$$

We now show (i). First let $\lambda \neq 0$. Since $y=w^{j}=\cos \frac{2 j}{n+1} \pi+i \sin \frac{2 j}{n+1} \pi$ for some $j, 1 \leq j \leq n$, by (7) and $y=\frac{\mu}{\lambda} \in \mathbb{Q}, \sin \frac{2 j}{n+1} \pi=0$. Hence $\frac{2 j \pi}{n+1}=0$ or $\frac{2 j \pi}{n+1}=\pi$. If $\frac{2 j}{n+1} \pi=0$, then $j=0$. This contradicts $1 \leq j \leq n$. If $\frac{2 j}{n+1} \pi=\pi$, then $j=\frac{n+1}{2}$. Since $n$ is even, $\frac{n+1}{2}$ is not an integer, but $j$ must be an integer. Hence $j=\frac{n+1}{2}$ is a contradiction. Next let $\lambda=0$. By (1), $\lambda=\mu=0$. Hence we have (i). 
Now, we consider (ii). If $\lambda=-\mu$, then

$$
\begin{aligned}
\lambda(f, g) & =\lambda^{n} \mu^{0}+\lambda^{n-1} \mu+\cdots+\lambda \mu^{n-1}+\lambda^{0} \mu^{n} \\
& =\sum_{j=0}^{\frac{n+1}{2}}\left(\lambda^{j} \mu^{n-j}+\lambda^{n-j} \mu^{j}\right)=\sum_{j=0}^{\frac{n+1}{2}}\left((-1)^{j}+(-1)^{n-j}\right) \lambda^{n} .
\end{aligned}
$$

Since $n$ is odd, $(-1)^{j}+(-1)^{n-j}=0$. Thus $\lambda(f, g)=0$.

We assume that $\lambda(f, g)=0$. First let $\lambda \neq 0$. Since $\cos \frac{2 j}{n+1} \pi+i \sin \frac{2 j}{n+1} \pi=$ $y=\frac{\mu}{\lambda} \in \mathbb{Q}$ for some $j, 1 \leq j \leq n$, by (7), $\sin \frac{2 \pi j}{n+1}=0$. Thus $\frac{2 \pi j}{n+1}=0$ or $\frac{2 \pi j}{n+1}=\pi$. If $\frac{2 \pi j}{n+1}=0$, then $j=0$. This contradicts $1 \leq j \leq n$. Hence $\frac{2 \pi j}{n+1}=\pi$, i.e., $j=\frac{n+1}{2}$. In this case $y=w^{j}=\cos \frac{1}{n+1} \cdot \frac{n+1}{2} \cdot 2 \pi+i \sin \frac{1}{n+1} \cdot \frac{n+1}{2} \cdot 2 \pi=$ $\cos \pi+i \sin \pi=-1$. Hence $y=-1$, i.e., $\frac{\mu}{\lambda}=-1$, so $\lambda=-\mu$. Next let $\lambda=0$. Then by (1) $\lambda=\mu=0$. Hence, we have (ii).

(6.3) Corollary. Let $\operatorname{deg}(f) \neq 0$.

(i) If $n$ is even, then $\lambda(f, g) \neq 0$ for any $g: P^{n}(\mathbb{C}) \rightarrow P^{n}(\mathbb{C})$.

(ii) If $n$ is odd, then $\lambda(f, g) \neq 0$ for any map $g: P^{n}(\mathbb{C}) \rightarrow P^{n}(\mathbb{C})$ with $\operatorname{deg}(g) \neq-\operatorname{deg}(f)$.

(6.4) Lemma. Let $n$ be even and $f: P^{n}(\mathbb{C}) \rightarrow P^{n}(\mathbb{C})$. If $\operatorname{deg}(f) \neq 0$, then $f$ is cosmic.

Proof. By (i) of Corollary (6.3), $\lambda(f, g) \neq 0$ for any $g$. By the coincidence point theorem of Lefschetz (see p. 320 of [L]), $f$ and $g$ have a coincidence point. Hence $f$ is cosmic.

(6.5) Lemma. Let $n$ be odd. If $\operatorname{deg}(f) \neq 0$, then $f$ and $g$ have a coincidence point for any $g: P^{n}(\mathbb{C}) \rightarrow P^{n}(\mathbb{C})$ with $\operatorname{deg}(g) \neq-\operatorname{deg}(f)$.

By (ii) of Corollary (6.3) and the Lefschetz coincidence point theorem [L] we have Lemma (6.5).

Let $[f]$ be the homotopy class of a map $f: P^{n}(\mathbb{C}) \rightarrow P^{n}(\mathbb{C})$, and let $\left[P^{n}(\mathbb{C}), P^{n}(\mathbb{C})\right]$ be the set of all homotopy classes of all maps between $P^{n}(\mathbb{C})$ and itself. Since $f_{*}: H_{2}\left(P^{n}(\mathbb{C}) ; \mathbb{Z}\right) \rightarrow H_{2}\left(P^{n}(\mathbb{C}) ; \mathbb{Z}\right)$ depends only on $[f]$, we define $\operatorname{deg}([f])$ by $\operatorname{deg}(f)$.

(6.6) Lemma. $\operatorname{deg}:\left[P^{n}(\mathbb{C}), P^{n}(\mathbb{C})\right] \rightarrow \mathbb{Z}$ is bijective.

To prove Lemma (6.6) we need some general results: Let $Y$ be an $(n-1)$ connected and $n$-simple space. By the universal coefficient theorem and the Hurewicz theorem, we have isomorphisms

$$
\begin{aligned}
H^{n}\left(Y ; \pi_{n}(Y)\right) & \stackrel{K}{\cong} \operatorname{Hom}\left(H_{n}(Y ; \mathbb{Z}), \pi_{n}(Y)\right), \\
\pi_{n}(Y) & \stackrel{E}{\cong} H_{n}(Y ; \mathbb{Z}) .
\end{aligned}
$$

Thus we may define a cohomology class $\gamma_{Y} \in H^{n}\left(Y ; \pi_{n}(Y)\right)$ by $K\left(\gamma_{Y}\right)=E^{-1}$. This cohomology class $\gamma_{Y}$ is called the $n$-characteristic cohomology class of $Y$.

(6.7) Lemma (Hopf-Eilenberg [Sp]). Let $X$ be a CW complex with $\operatorname{dim} X=$ $k$. Let $Y$ be $(n-1)$-connected and $j$-simple for $n \leq j \leq k$ such that $H^{j+1}\left(X ; \pi_{j}(Y)\right)=0$ for $n<j<k$, and $H^{j}\left(X ; \pi_{j}(Y)\right)=0$ for $n<j \leq k$. 
Then there is a bijection $\varphi:[X, Y] \rightarrow H^{n}\left(X ; \pi_{n}(Y)\right)$. This $\varphi$ is defined by $[f] \rightarrow f^{*}\left(\gamma_{Y}\right)$.

Proof of Lemma (6.6). In the first paragraph of this section we described algebraic data (1), (2), and (3) for $P^{n}(\mathbb{C})$. Using this data we can apply Lemma (6.7) with $X=Y=P^{n}(\mathbb{C}), n=2, k=2 n$. Thus we have a bijection

$$
\varphi:\left[P^{n}(\mathbb{C}), P^{n}(\mathbb{C})\right] \rightarrow H^{2}\left(P^{n}(\mathbb{C}) ; \pi_{2}\left(P^{n}(\mathbb{C})\right)\right),
$$

which is defined by $\varphi([f])=f^{*}(\gamma)$ for any $[f]$. Take any $f: P^{n}(\mathbb{C}) \rightarrow P^{n}(\mathbb{C})$. We have the commutative diagrams:

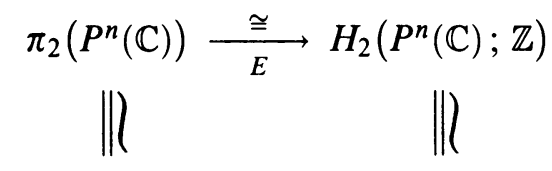

$\mathbb{Z}$

$\mathbb{Z}$

$$
\begin{array}{ccc}
H^{2}\left(P^{n}(\mathbb{C}) ; \pi_{2}\left(P^{n}(\mathbb{C})\right)\right) \stackrel{\cong}{\leftrightarrows} \operatorname{Hom}\left(H_{2}\left(P^{n}(\mathbb{C}) ; \mathbb{Z}\right), \pi_{2}\left(P^{n}(\mathbb{C})\right)\right) & \downarrow \operatorname{Hom}\left(f_{*}, 1\right) \\
\left\lfloor f^{*}\right. & & \cong \\
H^{2}\left(P^{n}(\mathbb{C}) ; \pi_{2}\left(P^{n}(\mathbb{C})\right)\right) & \left.\stackrel{\cong}{K} \operatorname{Hom}\left(H_{2}\left(P^{n}(\mathbb{C})\right) ; \mathbb{Z}\right), \pi_{2}\left(P^{n}(\mathbb{C})\right)\right) .
\end{array}
$$

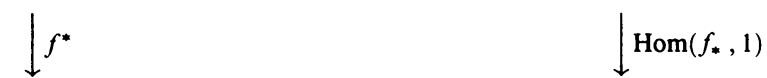

(3)

Let $\alpha$ and $\beta$ be generators of $H_{2}\left(P^{n}(\mathbb{C}) ; \mathbb{Z}\right)$ and $\pi_{2}\left(P^{n}(\mathbb{C})\right)$, respectively. Since $P^{n}(\mathbb{C})$ is simply connected, the Hurewicz homomorphism $E: \pi_{2}\left(P^{n}(\mathbb{C})\right.$ ) $=\mathbb{Z} \beta \rightarrow H_{2}\left(P^{n}(\mathbb{C}) ; \mathbb{Z}\right)=\mathbb{Z} \alpha$ is an isomorphism. Thus

$$
E(\beta)=( \pm 1) \alpha \text {. }
$$

However, when we replace a generator $\beta$ by $-\beta$, without loss of generality, we may assume that $E(\beta)=\alpha$, i.e.,

$$
E^{-1}(\alpha)=\beta
$$

Therefore, $E^{-1}: H_{2}\left(P^{n}(\mathbb{C}) ; \mathbb{Z}\right)=\mathbb{Z} \alpha \rightarrow \pi_{2}\left(P^{n}(\mathbb{C})\right)=\mathbb{Z} \beta$ is defined by $k \alpha \rightarrow$ $k \beta$ for any $k \in \mathbb{Z}$.

It is well known that for any homomorphism $h: \mathbb{Z} \alpha \rightarrow \mathbb{Z} \beta$, there exists a unique integer $\psi(h) \in \mathbb{Z}$ such that

$$
h(k \alpha)=\psi(h) k \beta
$$

for each $k \in \mathbb{Z}$.

For any integer $n_{0} \in \mathbb{Z}$, we define the homomorphism $n_{0} E^{-1}: \mathbb{Z} \alpha \rightarrow \mathbb{Z} \beta$ by

$$
n_{0} E^{-1}(k \alpha)=n_{0} k \beta \quad \text { for any } k \in \mathbb{Z} \text {. }
$$

Therefore, (6) and (7) mean that

$$
h=\psi(h) E^{-1} .
$$

Clearly,

$$
\psi: \operatorname{Hom}\left(H_{2}\left(P^{n}(\mathbb{C}) ; \mathbb{Z}\right), \pi_{2}\left(P^{n}(\mathbb{C})\right)\right) \rightarrow \mathbb{Z}
$$

is bijective. Moreover it is group isomorphic. By (8),

$$
\psi\left(E^{-1}\right)=1 .
$$


By (3), $K f^{*}(\gamma)=K f^{*} K^{-1}\left(E^{-1}\right)=\operatorname{Hom}\left(f_{*}, 1\right) K K^{-1}\left(E^{-1}\right)=\operatorname{Hom}\left(f_{*}, 1\right)\left(E^{-1}\right)$ $=E^{-1} f_{*}$, i.e.,

$$
K f^{*}(\gamma)=E^{-1} f_{*}
$$

By the definition and (5), $E^{-1} f_{*}(k \alpha)=E^{-1}((\operatorname{deg} f) k \alpha)=(\operatorname{deg} f) k \beta$, i.e.,

$$
E^{-1} f_{*}=(\operatorname{deg} f) E^{-1} \text {. }
$$

By (11) and (12), $\psi\left(K f^{*}(\gamma)\right)=\operatorname{deg} f$. Thus by (1),

$$
\psi K \varphi([f])=\operatorname{deg} f .
$$

This means that

$$
\psi K \varphi=\text { deg. }
$$

Since $\varphi, K$, and $\psi$ are bijective, by (14) deg is also bijective. Hence we have completed the proof of Lemma (6.6).

(6.8) Lemma. $\operatorname{deg}[g \circ f]=(\operatorname{deg}[g])(\operatorname{deg}[f])$ for any $f, g: P^{n}(\mathbb{C}) \rightarrow P^{n}(\mathbb{C})$.

This lemma is trivial by using the definition of deg.

(6.9) Lemma. Let $n$ be even. Let $X$ be a $P^{n}(\mathbb{C})$-like Hausdorff continuum. If $X$ is not movable, then it is cosmic.

Proof. Since $X$ is a $P^{n}(\mathbb{C})$-like Hausdorff continuum, by [MM] there exists an approximate resolution $\mathbf{p}=\left\{p_{a}: a \in A\right\}: X \rightarrow(\mathscr{X}, \mathscr{U})=\left\{X_{a}, \mathscr{U}_{a}, p_{a a^{\prime}}, A\right\}$ such that

(1) each $X_{a}$ is $P^{n}(\mathbb{C})$,

(2) $A$ is cofinite, antisymmetric, infinite and directed.

Since each $X_{a}$ is an ANR by (1), there exists a $\mathscr{V}_{a} \in \operatorname{Cov}\left(X_{a}\right), \mathscr{V}_{a}<\mathscr{W}_{a}$, with the property (see [HU]):

(3) For any space $Y$ and any maps $h, k: Y \rightarrow X_{a}$, if $(h, k)<s t^{4} \mathscr{V}_{a}$, then $h$ and $k$ are homotopic.

By (1.19) there exists an approximate resolution $\mathbf{q}=\left\{q_{a}: a \in A^{*}\right\}: X \rightarrow$ $(\mathscr{Y}, \mathscr{W})=\left\{Y_{a}, \mathscr{W}_{a}, q_{a a^{\prime}}, A^{*}\right\}$ such that

(4) $A^{*}$ is $A$ as sets,

(5) $Y_{a}, \mathscr{W}_{a}, q_{a a^{\prime}}$, and $q_{a}$ are $X_{a}, \mathscr{V}_{a}, p_{a a^{\prime}}$, and $p_{a}$ for each $a \in A^{*}$,

(6) $\left(q_{a}, q_{a a^{\prime}} q_{a^{\prime}}\right)<\mathscr{V}_{a}$ for any $a^{\prime} \gg a$.

By (1.20) and (3)-(5),

(7) $H(\mathbf{q}): X \rightarrow H(\mathscr{Y})$ is an HANR-expansion.

By (1) and (5), each $q_{a a^{\prime}}: Y_{a^{\prime}}=P^{n}(\mathbb{C}) \rightarrow P^{n}(\mathbb{C})=Y_{a}$ for $a^{\prime} \gg a$ has degree $\operatorname{deg}\left[q_{a a^{\prime}}\right]$. We show that

(8) there exists an $a_{0} \in A$ such that $\operatorname{deg}\left[q_{a_{0} a^{\prime}}\right] \neq 0$ for any $a^{\prime} \gg a_{0}$.

We assume that $(8)$ is not true. Then by $(8)$

(9) for each $a \in A$ there exists an $a^{\prime} \gg a$ such that $\operatorname{deg}\left[q_{a a^{\prime}}\right]=0$.

We claim that

(10) $H(\mathscr{Y})$ is movable.

To show (10) take any $a \in A^{*}$. By (9) there exists an $a^{\prime} \gg a$ such that $\operatorname{deg}\left[q_{a a^{\prime}}\right]=0$. Take any $a^{\prime \prime} \gg a$ and let $h: Y_{a^{\prime}} \rightarrow Y_{a^{\prime \prime}}$ be a constant map. Since $\operatorname{deg}[h]=0, \operatorname{deg}\left[q_{a a^{\prime \prime}} h\right]=\operatorname{deg}\left[q_{a a^{\prime \prime}}\right] \operatorname{deg}[h]=0=\operatorname{deg}\left[q_{a a^{\prime}}\right]$. Since $\operatorname{deg}\left[q_{a a^{\prime \prime}} h\right]=$ $\operatorname{deg}\left[q_{a a^{\prime}}\right]$, by $(6.6)$ 
(11) $\left[q_{a a^{\prime \prime}}\right][h]=\left[q_{a a^{\prime}}\right]$.

This means that $H(\mathscr{Y})$ is movable. So we have (10).

By (10), $X$ is movable. Thus it contradicts the assumption. Hence we have (8). Since $0 \neq \operatorname{deg}\left[q_{a_{0} a^{\prime \prime}}\right]=\operatorname{deg}\left[q_{a_{0} a^{\prime}}\right] \operatorname{deg}\left[q_{a^{\prime} a^{\prime \prime}}\right]$ and $\operatorname{deg}\left[q_{a_{0} a^{\prime}}\right] \neq 0$ for $a^{\prime \prime} \gg a^{\prime} \gg a$ by $(8), \operatorname{deg}\left[q_{a^{\prime} a^{\prime \prime}}\right] \neq 0$. Thus by $(8)$

(12) there exists an $a_{0} \in A^{*}$ such that $\operatorname{deg}\left[q_{a^{\prime} a^{\prime \prime}}\right] \neq 0$ for any $a^{\prime \prime} \gg a^{\prime} \gg a_{0}$. Let $A^{*}\left(a_{0}\right)=\left\{a \in A: a \gg a_{0}\right\}$. Since $\mathbf{q}: X \rightarrow(\mathscr{Y}, \mathscr{W})$ is an approximate resolution and $A^{*}\left(a_{0}\right)$ is a cofinal subset of $A^{*}$, it is easy to show that

(13) $\mathbf{q}^{*}=\left\{q_{a}: a \in A^{*}\left(a_{0}\right)\right\}: X \rightarrow(\mathscr{Y}, \mathscr{W})^{*}=\left\{Y_{a}, \mathscr{W}_{a}, q_{a a^{\prime}}, A^{*}\left(a_{0}\right)\right\}$ is an approximate resolution.

Since $\operatorname{deg}\left[q_{a^{\prime} a^{\prime \prime}}\right] \neq 0$ for each $a^{\prime \prime} \gg a^{\prime} \gg a_{0}$ by (12), $q_{a^{\prime} a^{\prime \prime}}$ and any map $h$ : $Y_{a^{\prime \prime}} \rightarrow Y_{a^{\prime}}$ have a coincidence point. Thus, $(\mathscr{Y}, \mathscr{W})^{*}$ satisfies condition (AC). Hence, by (13), $X$ is cosmic.

(6.10) Corollary. Let $n$ be even. Let $X$ be a $P^{n}(\mathbb{C})$-like Hausdorff continuum. If $X$ is not movable, then it has the fixed point property.

Corollary (6.10) follows from (5.13) and (6.9).

(6.11) Lemma. Let $n$ be even. Let $X$ be a $P^{n}(\mathbb{C})$-like Hausdorff continuum. If $X$ has the shape of $P^{n}(\mathbb{C})$, then it satisfies $(\mathrm{AC})$ and hence has the fixed point property.

Proof. In the same way as above, we can obtain an approximate system $(\mathscr{X}, \mathscr{U})$ $=\left\{X_{a}, \mathscr{U}_{a}, f_{a a^{\prime}}, A\right\}$ satisfying (1), (2), (3), (4) in the proof of Lemma (6.9), and

$$
\operatorname{deg}\left[f_{a a^{\prime}}\right]=1 \text { for all } a \leq a^{\prime} .
$$

In the same way as in Lemma (6.9), we can show that $(\mathscr{X}, \mathscr{U})$ satisfies (AC). Hence $X$ has the fixed point property by (5.13).

Watanabe [W4] has shown that there is only one other shape class for a $P^{n}(\mathbb{C})$-like Hausdorff continuum $X$ to consider, namely the case when $X$ is of trivial shape.

(6.12) Question. Let $n$ be even. Let $X$ be a $P^{n}(\mathbb{C})$-like Hausdorff continuum. If $X$ has the trivial shape, does it have the fixed point property? (See (7.13) for some partial results for this question.)

(6.13) Remark. Note that for $n=1, P^{n}(\mathbb{C})=S^{2}$. So one can readily see that (6.9), (6.10), and (6.11) are all false in this case. For the first two cases consider $X$ to be the suspension of the Dyadic Solenoid and for the third consider $X$ to be $S^{2}$.

\section{NEARLY EXTENDABLE MAPS AND APPROXIMATE RESOLUTIONS}

In [Bo2] Borsuk introduced the notion of a nearly extendable map between compact metric spaces. This notion was extended to a map between arbitrary spaces by Watanabe [W3]. He used approximate resolutions in his sense, i.e., commutative approximate resolutions in our sense. Thus, we shall give a definition of a nearly extendable map by using approximate resolutions in our sense, i.e., by using noncommutative approximate resolutions.

Let $\mathbf{f}=\left\{f, f_{b}: b \in B\right\}:(\mathscr{X}, \mathscr{U})=\left\{X_{a}, \mathscr{U}_{a}, p_{a a^{\prime}}, A\right\} \rightarrow(\mathscr{Y}, \mathscr{V})=$ $\left\{Y_{b}, \mathscr{V}_{b}, q_{b b^{\prime}}, B\right\}$ be an approximate map. We consider the following condition: 
(NE)* For each $b \in B$ there exists an $a_{0}>f(b)$ with two properties:

(i) $\left(p_{f(b) a_{0}} p_{a_{0} a^{\prime}}, p_{f(b) a^{\prime}}\right)<f_{b}^{-1} \mathscr{V}_{b}$ for any $a^{\prime}>a_{0}$.

(ii) There exists a map $h: X_{a_{0}} \rightarrow Y_{b^{\prime}}$ such that $\left(f_{b} p_{f(b) a_{0}}, q_{b b^{\prime}} h\right)<\mathbf{s t}^{2} \mathscr{V}_{b}$ for any $b^{\prime}>b$.

(7.1) Lemma. If $\mathbf{f}$ is a uniform approximate map, then it satisfies property (i) in $(\mathrm{NE}) *$.

Proof. By (A2) for $(\mathscr{X}, \mathscr{U})$, for each $b \in B$, there exists an $a_{0}>f(b)$ such that

$$
\left(p_{f(b) a_{0}} p_{a_{0} a^{\prime}}, p_{f(b) a^{\prime}}\right)<\mathscr{U}_{f(b)} \text { for any } a^{\prime}>a_{0}>f(b) .
$$

Since $\mathbf{f}$ is uniform,

$$
f_{b}^{-1} \mathscr{V}_{b}>\mathscr{U}_{f(b)} .
$$

By (1) and (2), we have property (i).

(7.2) Lemma. Let $(\mathscr{X}, \mathscr{U})$ and $(\mathscr{Y}, \mathscr{V})$ be commutative approximate systems. If $\mathbf{f}:(\mathscr{X}, \mathscr{U}) \rightarrow(\mathscr{Y}, \mathscr{V})$ is uniform, then the condition $(\mathrm{NE}) *$ is equivalent to condition (NE) in [W3].

Proof. By Lemma (7.1), we need only property (ii) in (NE)*. Thus, clearly the condition (NE) implies the condition (NE)*.

We show that (NE)* implies (NE). Take any $b \in B$. By (AI3) (see [W1]) there exists a $b_{1}>b$ such that

$$
q_{b b_{1}}^{-1} \mathscr{V}_{b}>\mathrm{st}^{2} \mathscr{V}_{b_{1}} \text {. }
$$

By the assumption, there exists an $a_{0}>f\left(b_{1}\right)$ satisfying (NE)* for $b_{1}$. Since $b_{1}>b$, by (AM2) of [W1] there exists an $a_{1}>a_{0}, f(b)$ such that

$$
\left(f_{b} p_{f(b) a_{1}}, q_{b b_{1}} f_{b_{1}} p_{f\left(b_{1}\right) a_{1}}\right)<\mathscr{V}_{b} .
$$

Take any $b^{\prime}>b$. Take any $b^{\prime \prime}>b^{\prime}, b_{1}$. By the choice of $a_{0}$, there exists a map $h: X_{a_{0}} \rightarrow Y_{b^{\prime \prime}}$ such that

$$
\left(q_{b_{1} b^{\prime \prime}} h, f_{b_{1}} p_{f\left(b_{1}\right) a_{0}}\right)<\mathbf{s t}^{2} \mathscr{V}_{b_{1}}
$$

Since $(\mathscr{Y}, \mathscr{V})$ is commutative, by (1) and (3),

$$
\left(q_{b b^{\prime \prime}} h, q_{b b_{1}} f_{b_{1}} p_{f\left(b_{1}\right) a_{0}}\right)<\mathscr{V}_{b} .
$$

Since $(\mathscr{X}, \mathscr{U})$ is commutative, by $(4)$,

$$
\left(q_{b b^{\prime}} q_{b^{\prime} b^{\prime \prime}} h p_{a_{0} a_{1}}, q_{b b_{1}} f_{b_{1}} p_{f\left(b_{1}\right) a_{1}}\right)<\mathscr{V}_{b} .
$$

We put $\tilde{h}=q_{b^{\prime} b^{\prime \prime}} h p_{a_{0} a_{1}}: X_{a_{1}} \rightarrow Y_{b^{\prime}}$. By (2) and (5),

$$
\left(q_{b b}, \tilde{h}, f_{b} p_{f(b) a_{1}}\right)<\mathrm{st} \mathscr{V}_{b} \text {. }
$$

Thus we have the condition (NE).

(7.3) Corollary. Let $\mathbf{f}:(\mathscr{X}, \mathscr{U}) \rightarrow(\mathscr{Y}, \mathscr{V})$ be an approximate system map in the sense of [W1]. Then $\mathbf{f}$ satisfies the condition (NE) if and only if it satisfies condition (NE)*.

We can prove the following lemma by a slight modification of the proof of (1.1) in [W3]. 
(7.4) Lemma. Let $\mathbf{f}, \mathbf{g}:(\mathscr{X}, \mathscr{U}) \rightarrow(\mathscr{Y}, \mathscr{V})$ be approximate system maps. If $\mathbf{f} \sim \mathbf{g}$ and $\mathbf{f}$ satisfies condition $(\mathrm{NE}) *$, then so does $\mathbf{g}$.

By Lemma (7.4), we can say that [f] satisfies the condition (NE)* provided f satisfies condition $(\mathrm{NE})^{*}$. We can prove the following lemma by a slight modification of the proof of (1.2) in [W3].

(7.5) Lemma. Let $\mathbf{f}:(\mathscr{X}, \mathscr{U}) \rightarrow(\mathscr{Y}, \mathscr{V})$ and $\mathbf{g}:(\mathscr{Y}, \mathscr{V}) \rightarrow(\mathscr{Z}, \mathscr{W})$ be approximate system maps.

(i) If [f] satisfies $(\mathrm{NE})^{*}$, then so does [g][f].

(ii) If $[\mathbf{g}]$ satisfies $(\mathrm{NE}) *$, then so does $[\mathbf{g}][\mathbf{f}]$.

Let $f: X \rightarrow Y$ be a map. Let $\mathbf{p}: X \rightarrow(\mathscr{X}, \mathscr{U}), \mathbf{p}^{\prime}: X \rightarrow(\mathscr{X}, \mathscr{U})^{\prime}, \mathbf{q}:$ $Y \rightarrow(\mathscr{Y}, \mathscr{V})$, and $\mathbf{q}^{\prime}: Y \rightarrow(\mathscr{Y}, \mathscr{V})^{\prime}$ be approximate APOL-resolutions. Let $\mathbf{f}:(\mathscr{X}, \mathscr{U}) \rightarrow(\mathscr{Y}, \mathscr{V})$ and $\mathbf{f}^{\prime}:(\mathscr{X}, \mathscr{U})^{\prime} \rightarrow(\mathscr{Y}, \mathscr{V})^{\prime}$ be approximate resolutions of $f$.

(7.6) Lemma. $\mathbf{f}$ satisfies (NE)* if and only if $\mathbf{f}^{\prime}$ does.

Proof. Let i : $(\mathscr{X}, \mathscr{U}) \rightarrow(\mathscr{X}, \mathscr{U})^{\prime}$, and $\mathbf{i}^{\prime}:(\mathscr{X}, \mathscr{U})^{\prime} \rightarrow(\mathscr{X}, \mathscr{U})$ be approximate resolutions of $1_{X}: X \rightarrow X$. Let $\mathbf{j}:(\mathscr{Y}, \mathscr{V}) \rightarrow(\mathscr{Y}, \mathscr{V})^{\prime}$ and $\mathbf{j}^{\prime}:(\mathscr{Y}, \mathscr{V})^{\prime} \rightarrow(\mathscr{Y}, \mathscr{V})$ be approximate resolutions of $1_{Y}: Y \rightarrow Y$. Thus $\left[\mathbf{f}^{\prime}\right]=[\mathbf{j}][\mathbf{f}]\left[\mathbf{i}^{\prime}\right]$ and $[\mathbf{f}]=\left[\mathbf{j}^{\prime}\right]\left[\mathbf{f}^{\prime}\right][\mathbf{i}]$ by Corollary (1.17). So (7.6) follows from Lemma (7.5).

We say that a map $f: X \rightarrow Y$ is nearly extendable (or is an NE-map) provided any/some approximate resolution satisfies (NE)*. So (7.5) implies

(7.7) Lemma. Let $f: X \rightarrow Y, g: Y \rightarrow Z$ be maps.

(i) If $f$ is an NE-map, then so is $g f$.

(ii) If $g$ is an NE-map, then so is $g f$.

A space $X$ is an $N E$-set provided $1_{X}: X \rightarrow X$ is an NE-map. It is easy to show the following:

(7.8) Lemma. A space $X$ is an NE-set if and only if any/some approximate APOL-resolution $\mathbf{p}: X \rightarrow(\mathscr{X}, \mathscr{U})$ satisfies the condition:

(**) For each $a \in A$ there exists an $a_{0}>a$ such that for any $a^{\prime}>a$ there exists a map $h: X_{a_{0}} \rightarrow X_{a^{\prime}}$ with $\left(p_{a a_{0}}, p_{a a^{\prime}} h\right)<\mathrm{st}^{2} \mathscr{U}_{a}$.

Now, this condition $(* *)$ is equivalent to approximate movability.

(7.9) Lemma ((1.11) of [W3]). A space $X$ is an NE-set if and only if $X$ is approximately movable.

(7.10) Theorem. Let $\mathbf{p}: X \rightarrow(\mathscr{X}, \mathscr{U})$ be an approximate APOL-resolution of a compact Hausdorff space $X$. We assume that all terms $X_{a}$ have the fixed point property. If $f: X \rightarrow X$ is an NE-map, then $f$ has a fixed point.

Proof. Let $\mathbf{f}=\left\{f, f_{a}: a \in A\right\}:(\mathscr{X}, \mathscr{U}) \rightarrow(\mathscr{X}, \mathscr{U})$ be an approximate resolution of $f$. Take any $a \in A$. By (A3) there exists $a_{0}>a$ such that

$$
p_{a a^{\prime}}^{-1} \mathscr{U}_{a}>\mathbf{s t}^{3} \mathscr{U}_{a^{\prime}} \text { for any } a^{\prime}>a_{0} \text {. }
$$

By the assumption, there exists an $a_{1}>a_{0}, f\left(a_{0}\right)$ satisfying (i) and (ii) in $(\mathrm{NE})^{*}$ for $a_{0}$ and $\mathbf{f}$. 
Take any $a^{\prime}>a_{1}$. By the choice of $a_{1}$, there exists a map $h: X_{a_{1}} \rightarrow X_{a^{\prime}}$ such that

$$
\begin{gathered}
\left(p_{f\left(a_{0}\right) a_{1}} p_{a_{1} a^{\prime}}, \quad p_{f\left(a_{0}\right) a^{\prime}}\right)<f_{a_{0}}^{-1} \mathscr{U}_{a_{0}}, \\
\left(f_{a_{0}} p_{f\left(a_{0}\right) a_{1}}, \quad p_{a_{0} a^{\prime}} h\right)<\mathbf{s t}^{2} \mathscr{U}_{a_{0}} .
\end{gathered}
$$

Since $X_{a^{\prime}}$ has the fixed point property, there exists a point $x_{0} \in X_{a^{\prime}}$ such that

$$
h p_{a_{1} a^{\prime}}\left(x_{0}\right)=x_{0}
$$

By (2) and (3),

$$
\left(f_{a_{0}} p_{f\left(a_{0}\right) a^{\prime}}, p_{a_{0} a^{\prime}} h p_{a_{1} a^{\prime}}\right)<\mathbf{s t}^{3} \mathscr{U}_{a_{0}}
$$

By (1) and (5),

$$
\left(p_{a a_{0}} f_{a_{0}} p_{f\left(a_{0}\right) a^{\prime}}, \quad p_{a a_{0}} p_{a_{0} a^{\prime}} h p_{a_{1} a^{\prime}}\right)<\mathscr{U}_{a} .
$$

Since $p_{a a_{0}} p_{a_{0} a^{\prime}} h p_{a_{1} a^{\prime}}\left(x_{0}\right)=p_{a a_{0}} p_{a_{0} a^{\prime}}\left(x_{0}\right)$ by (4), by (6) there exists a $U \in \mathscr{U}_{a}$ such that

$$
p_{a a_{0}} f_{a_{0}} p_{f\left(a_{0}\right) a^{\prime}}\left(x_{0}\right), \quad p_{a a_{0}} p_{a_{0} a^{\prime}}\left(x_{0}\right) \in U .
$$

This means that f satisfies (AFP). Then, by Corollary (3.2), $f: X \rightarrow X$ has a $\mathscr{U}$-fixed point for any $\mathscr{U} \in \operatorname{Cov}(X)$. Hence, by Lemma (5.10), $f$ has a fixed point.

(7.11) Corollary. Let $X$ be an approximately movable Hausdorff compactum. If $X$ has an approximate resolution of polyhedra having the fixed point property, then $X$ has the fixed point property.

Corollary (7.11) follows from (7.9), (7.7), and (7.10).

(7.12) Remark. Without approximate movability, (7.11) does not hold. For example, the continua mentioned in Remark (5.18) have approximate resolutions with polyhedra each having the fixed point property, but do not have the fixed point property.

(7.13) Remark. In [W3] we proved a Lefschetz-type fixed point theorem for NE-maps. So under the conditions of Question (6.12) any NE-map has a fixed point. In the special case when $X$ is approximately movable, $X$ has the fixed point property by Lemma (7.9).

\section{REFINABLE MAPS AND COSMICALITY}

Let $(\mathscr{X}, \mathscr{U})=\left\{X_{a}, \mathscr{U}_{a}, p_{a a^{\prime}}, A\right\},(\mathscr{Y}, \mathscr{V})=\left\{Y_{b}, \mathscr{V}_{b}, q_{b b^{\prime}}, B\right\}$, and $(\mathscr{Z}, \mathscr{W})=\left\{Z_{c}, \mathscr{W}_{c}, r_{c c^{\prime}}, C\right\}$ be approximate inverse systems over cofinite directed sets. Let $\mathbf{f}=\left\{f, f_{b}: b \in B\right\}:(\mathscr{X}, \mathscr{U}) \rightarrow(\mathscr{Y}, \mathscr{V})$ be a system map. We say that $\mathbf{f}$ is an approximate pseudo-isomorphism provided it satisfies the following condition:

$$
\begin{aligned}
& (\forall b \in B)\left(\exists a_{0}>f(b)\right)\left(\forall a^{\prime}>a_{0}\right) \\
& \left(\exists b^{\prime}>b\right)\left(\exists g: Y_{b^{\prime}} \rightarrow X_{a^{\prime}}\right)\left(f_{b} p_{f(b) a^{\prime}} g, q_{b b^{\prime}}\right)<\mathrm{st}^{2} \mathscr{V}_{b} \\
& \left(\forall b^{\prime \prime}>b^{\prime}\right)\left(\exists a^{\prime \prime}>a^{\prime}\right)\left(\exists h: X_{a^{\prime \prime}} \rightarrow Y_{b^{\prime \prime}}\right) \\
& \left(g q_{b^{\prime} b^{\prime \prime}} h, p_{a^{\prime} a^{\prime \prime}}\right)<\mathrm{st} \mathscr{U}_{a^{\prime}} .
\end{aligned}
$$


(8.1) Lemma. Let $\mathbf{f}, \mathbf{f}^{\prime}=\left\{f^{\prime}, f_{b}^{\prime}: b \in B\right\}:(\mathscr{X}, \mathscr{U}) \rightarrow(\mathscr{Y}, \mathscr{V})$ be approximate system maps. Let $\mathbf{f} \sim \mathbf{f}^{\prime}$. If $\mathbf{f}$ is an approximate pseudo-isomorphism, then so is $\mathbf{f}^{\prime}$.

Proof. Without loss of generality we may assume that $\mathbf{f} \equiv \mathbf{f}^{\prime}$. Take any $b_{1} \in B$. By (A3) there exists a $b>b_{1}$ such that

$$
q_{b_{1} b}^{-1} \mathscr{V}_{b_{1}}>\mathrm{st}^{2} \mathscr{V}_{b}
$$

There exists an $a_{0}>f(b)$ satisfying (API) for $\mathbf{f}$ and $b$. Since $\mathbf{f} \equiv \mathbf{f}^{\prime}$, by (AM) for $\mathbf{f}^{\prime}$ and (EM) of [MW] there exists an $a_{1}>f^{\prime}\left(b_{1}\right), f^{\prime}(b), a_{0}$ such that

$$
\left(f_{b_{1}}^{\prime} p_{f^{\prime}\left(b_{1}\right) a^{\prime}}, q_{b_{1} b} f_{b}^{\prime} p_{f^{\prime}(b) a^{\prime}}\right)<\text { st } \mathscr{V}_{b_{1}}
$$

for any $a^{\prime}>a_{1}$ and

$$
\left(f_{b} p_{f(b) a^{\prime}}, f_{b}^{\prime} p_{f^{\prime}(b) a^{\prime}}\right)<\text { st } \mathscr{V}_{b}
$$

for any $a^{\prime}>a_{1}$.

Take any $a^{\prime}>a_{1}$. By the choice of $a_{0}$ there exist a $b^{\prime}>b$ and a map $g: Y_{b^{\prime}} \rightarrow X_{a^{\prime}}$ satisfying (API). Then

$$
\left(f_{b} p_{f(b) a^{\prime}} g, q_{b b^{\prime}}\right)<\mathrm{st}^{2} \mathscr{V}_{b} \text {. }
$$

Take any $b^{\prime \prime}>b^{\prime}$. By (API) and the choices of $b^{\prime}$ and $g$, there exist an $a^{\prime \prime}>a^{\prime}$ and a map $h: X_{a^{\prime \prime}} \rightarrow Y_{b^{\prime \prime}}$ such that

$$
\left(g q_{b^{\prime} b^{\prime \prime}} h, p_{a^{\prime} a^{\prime \prime}}\right)<\mathrm{st} \mathscr{U}_{a^{\prime}} \text {. }
$$

By (1) and (4),

$$
\left(q_{b_{1} b} f_{b} p_{f(b) a^{\prime}} g, q_{b_{1} b} q_{b b^{\prime}}\right)<\mathscr{V}_{b_{1}} .
$$

By (1) and (3),

$$
\left(q_{b_{1} b} f_{b} p_{f(b) a^{\prime}} g, q_{b_{1} b} f_{b}^{\prime} p_{f^{\prime}(b) a^{\prime}} g\right)<\mathscr{V}_{b_{1}}
$$

By (2),

$$
\left(f_{b_{1}}^{\prime} p_{f^{\prime}\left(b_{1}\right) a^{\prime}} g, q_{b_{1} b} f_{b}^{\prime} p_{f^{\prime}(b) a^{\prime}} g\right)<\mathrm{st} \mathscr{V}_{b_{1}} .
$$

Since $\left(q_{b_{1} b} q_{b b^{\prime}}, q_{b_{1} b^{\prime}}\right)<\mathscr{V}_{b_{1}}$ by (A1), by (6), (7), and (8),

$$
\left(q_{b_{1} b^{\prime}}, f_{b_{1}}^{\prime} p_{f^{\prime}\left(b_{1}\right) a^{\prime}} g\right)<\mathrm{st}^{2} \mathscr{V}_{b_{1}} \text {. }
$$

Inequalities (9) and (5) mean that $\mathbf{f}^{\prime}$ satisfies (API).

By (8.1) we can say that [f] is an approximate pseudo-isomorphism provided $f$ is an approximate pseudo-isomorphism.

(8.2) Lemma. Let $\mathbf{f}:(\mathscr{X}, \mathscr{U}) \rightarrow(\mathscr{Y}, \mathscr{V})$ and $\mathbf{g}:(\mathscr{Y}, \mathscr{V}) \rightarrow(\mathscr{Z}, \mathscr{W})$ be approximate system maps. If [f] and [g] are approximate pseudo-isomorphisms, then so is $[\mathbf{g}][\mathbf{f}]$.

Proof. By Lemma (8.1) and (8.6) of [MW] we may assume that $\mathbf{f}$ and $\mathbf{g}$ are uniform. Moreover, by (8.2) of [MW] we may assume that

$$
\text { gf : }(\mathscr{X}, \mathscr{U}) \rightarrow(\mathscr{Z}, \mathscr{W}) \text { is an approximate map }
$$

and

$$
[\mathbf{g f}]=[\mathbf{g}][\mathbf{f}]
$$


We show that gf satisfies (API). Take any $c_{1} \in C$. By (A3) there exists a $c_{2}>c_{1}$ such that

$$
r_{c_{1} c^{\prime}}^{-1} \mathscr{W}_{c_{1}}>\mathrm{st}^{4} \mathscr{W}_{c^{\prime}} \quad \text { for any } c^{\prime}>c_{2} \text {. }
$$

By (1) and (AM), there exists an $a_{1}>f g\left(c_{1}\right), f g\left(c_{2}\right)$ such that

(4) $\quad\left(g_{c_{1}} f_{g\left(c_{1}\right)} p_{f g\left(c_{1}\right) a^{\prime}}, r_{c_{1} c_{2}} g_{c_{2}} f_{g\left(c_{2}\right)} p_{f g\left(c_{2}\right) a^{\prime}}\right)<$ st $\mathscr{W}_{c_{1}}$ for any $a^{\prime}>a_{1}$.

Since $\mathbf{f}$ and $\mathbf{g}$ satisfy (API), there exist a $b_{1}>g\left(c_{2}\right)$ and an $a_{2}>f g\left(c_{2}\right)$ satisfying (API) for $\mathbf{g}, c_{2}$, and for $\mathbf{f}, g\left(c_{2}\right)$.

There exists an $a_{3}>a_{1}, a_{2}$. Take any $a^{\prime}>a_{3}$. By (A3) there exists an $a_{4}>a^{\prime}$ such that

$$
p_{a^{\prime} a^{\prime \prime}}^{-1} \mathscr{U}_{a^{\prime}}>\mathrm{st}^{2} \mathscr{U}_{a^{\prime \prime}} \text { for any } a^{\prime \prime}>a_{4} .
$$

Then $a_{4}>a_{2}$, so by the choice of $a_{2}$, there exist a $b_{2}>g\left(c_{2}\right)$ and a map $\alpha: Y_{b_{2}} \rightarrow X_{a_{4}}$ satisfying (API). Then

$$
\left(f_{g\left(c_{2}\right)} p_{f g\left(c_{2}\right) a_{4}} \alpha, q_{g\left(c_{2}\right) b_{2}}\right)<\operatorname{st}^{2} \mathscr{V}_{g\left(c_{2}\right)} .
$$

By (A2) and (A3) there exists a $b_{3}>b_{1}, b_{2}$ such that

$$
q_{b_{2} b^{\prime}}^{-1}\left(\alpha^{-1} \mathscr{U}_{a_{4}}\right)>\text { st } \mathscr{V}_{b^{\prime}} \text { for any } b^{\prime}>b_{3},
$$

$$
\left(q_{b_{2} b_{3}} q_{b_{3} b^{\prime \prime}}, q_{b_{2} b^{\prime \prime}}\right)<\alpha^{-1} \mathscr{U}_{a_{4}} \text { for any } b^{\prime \prime}>b_{3} .
$$

By (API) for $\mathbf{g}$ and the choice of $b_{1}$, there exist a $c_{3}>c_{2}$ and a map $\beta$ : $Z_{c_{3}} \rightarrow Y_{b_{3}}$ satisfying (API). Thus

$$
\left(g_{c_{2}} q_{g\left(c_{2}\right) b_{3}} \beta, r_{c_{2} c_{3}}\right)<\mathrm{st}^{2} \mathscr{W}_{c_{2}} .
$$

Take any $c^{\prime \prime}>c_{3}$. By (API) for $\mathbf{f}$ and $\mathbf{g}$, there exist a $b^{\prime \prime}>b_{3}$, an $a^{\prime \prime}>a_{4}$, and maps $\gamma: Y_{b^{\prime \prime}} \rightarrow Z_{c^{\prime \prime}}, \delta: X_{a^{\prime \prime}} \rightarrow Y_{b^{\prime \prime}}$ such that

$$
\begin{aligned}
& \left(\beta r_{c_{3} c^{\prime \prime}} \gamma, q_{b_{3} b^{\prime \prime}}\right)<\text { st } \mathscr{V}_{b_{3}}, \\
& \left(\alpha q_{b_{2} b^{\prime \prime}} \delta, p_{a_{4} a^{\prime \prime}}\right)<\text { st } \mathscr{U}_{a_{4}} .
\end{aligned}
$$

By (6),

$$
\left(f_{g\left(c_{2}\right)} p_{f g\left(c_{2}\right) a_{4}} \alpha q_{b_{2} b_{3}} \beta, q_{g\left(c_{2}\right) b_{2}} q_{b_{2} b_{3}} \beta\right)<\mathrm{st}^{2} \mathscr{V}_{g\left(c_{2}\right)}
$$

By (A1),

$$
\left(q_{g\left(c_{2}\right) b_{2}} q_{b_{2} b_{3}} \beta, q_{g\left(c_{2}\right) b_{3}} \beta\right)<\mathscr{V}_{g\left(c_{2}\right)} .
$$

Since $\mathbf{g}$ is uniform,

$$
g_{c_{2}}^{-1} \mathscr{W}_{c_{2}}>\mathscr{V}_{g\left(c_{2}\right)}
$$

By (12), (13), and (14),

$$
\left(g_{c_{2}} f_{g\left(c_{2}\right)} p_{f g\left(c_{2}\right) a_{4}} \alpha q_{b_{2} b_{3}} \beta, g_{c_{2}} q_{g\left(c_{2}\right) b_{3}} \beta\right)<\mathrm{st}^{3} \mathscr{W}_{c_{2}} .
$$

By (9), (15), (3), and (A1),

$$
\left(r_{c_{1} c_{2}} g_{c_{2}} f_{g\left(c_{2}\right)} p_{f g\left(c_{2}\right) a_{4}} \alpha q_{b_{2} b_{3}} \beta, r_{c_{1} c_{3}}\right)<\text { st } \mathscr{W}_{c_{1}} .
$$

By (4),

$$
\left(g_{c_{1}} f_{g\left(c_{1}\right)} p_{f g\left(c_{1}\right) a_{4}} \alpha q_{b_{2} b_{3}} \beta, r_{c_{1} c_{2}} g_{c_{2}} f_{g\left(c_{2}\right)} p_{f g\left(c_{2}\right) a_{4}} \alpha q_{b_{2} b_{3}} \beta\right)<\text { st } \mathscr{W}_{c_{1}} .
$$


Since $\left(p_{f g\left(c_{1}\right) a^{\prime}} p_{a^{\prime} a_{4}}, p_{f g\left(c_{1}\right) a_{4}}\right)<\mathscr{U}_{f g\left(c_{1}\right)}$ by $(\mathrm{A} 1)$ and $\left(g_{c_{1}} f_{g\left(c_{1}\right)}\right)^{-1} \mathscr{W}_{c_{1}}>\mathscr{U}_{f g\left(c_{1}\right)}$ by uniformities of $\mathbf{f}$ and $\mathbf{g}$,

$$
\left(g_{c_{1}} f_{g\left(c_{1}\right)} p_{f g\left(c_{1}\right) a^{\prime}} p_{a^{\prime} a_{4}} \alpha q_{b_{2} b_{3}} \beta, g_{c_{1}} f_{g\left(c_{1}\right)} p_{f g\left(c_{1}\right) a_{4}} \alpha q_{b_{2} b_{3}} \beta\right)<\mathscr{W}_{c_{1}} .
$$

By (16), (17), and (18),

$$
\left(g_{c_{1}} f_{g\left(c_{1}\right)} p_{f g\left(c_{1}\right) a^{\prime}} p_{a^{\prime} a_{4}} \alpha q_{b_{2} b_{3}} \beta, r_{c_{1} c_{3}}\right)<\mathrm{st}^{2} \mathscr{W}_{c_{1}} .
$$

By (10) and (7),

$$
\left(\alpha q_{b_{2} b_{3}} \beta r_{c_{3} c^{\prime \prime}} \gamma \delta, \alpha q_{b_{2} b_{3}} q_{b_{3} b^{\prime \prime}} \delta\right)<\mathscr{U}_{a_{4}}
$$

By (8),

$$
\left(\alpha q_{b_{2} b_{3}} q_{b_{3} b^{\prime \prime}} \delta, \alpha q_{b_{2} b^{\prime \prime}} \delta\right)<\mathscr{U}_{a_{4}} .
$$

By (20), (21), (11), and (5),

$$
\left(p_{a^{\prime} a_{4}} \alpha q_{b_{2} b_{3}} \beta r_{c_{3} c^{\prime \prime}} \gamma \delta, p_{a^{\prime} a_{4}} p_{a^{\prime} a^{\prime \prime}}\right)<\mathscr{U}_{a^{\prime}} .
$$

Since $\left(p_{a^{\prime} a_{4}} p_{a_{4} a^{\prime \prime}}, p_{a^{\prime} a^{\prime \prime}}\right)<\mathscr{U}_{a^{\prime}}$ by (A1), by (22) we have

$$
\left(p_{a^{\prime} a_{4}} \alpha q_{b_{2} b_{3}} \beta r_{c_{3} c^{\prime \prime}} \gamma \delta, p_{a^{\prime} a^{\prime \prime}}\right)<\text { st } \mathscr{U}_{a^{\prime}} \text {. }
$$

Inequalities (19) and (23) mean that $m=p_{a^{\prime} a_{4}} \alpha q_{b_{2} b_{3}} \beta: Z_{c_{3}} \rightarrow X_{a^{\prime}}$ and $n=$ $\gamma \delta: X_{a^{\prime \prime}} \rightarrow Z_{c^{\prime \prime}}$ satisfy the condition (API) for $\mathbf{g f}$.

(8.3) Lemma. If [f] : $(\mathscr{X}, \mathscr{U}) \rightarrow(\mathscr{Y}, \mathscr{V})$ is an isomorphism in APRO-TOP, then [f] is an approximate pseudo-isomorphism.

Proof. Since [f] is an isomorphism, there exists a morphism [g]: $(\mathscr{Y}, \mathscr{V}) \rightarrow$ $(\mathscr{X}, \mathscr{U})$ such that

$$
[\mathbf{g}][\mathbf{f}]=\left[1_{(\mathscr{Z}, \mathscr{U})}\right] \text { and }[\mathbf{f}][\mathbf{g}]=\left[1_{(\mathscr{Y}, \mathscr{V})}\right] \text {. }
$$

By (8.6) of [MW] and (8.1) we may assume that $f$ and $g$ are uniform. Then by (8.2) of [MW] there are 2-refinement functions $s: A \rightarrow A$ and $s^{\prime}: B \rightarrow B$ such that

$$
[\mathbf{g}][\mathbf{f}]=[s(\mathbf{g}) \mathbf{f}] \text { and }[\mathbf{f}][\mathbf{g}]=\left[s^{\prime}(\mathbf{f}) \mathbf{g}\right] \text {. }
$$

By (7.8) of [MW] there exist refinement functions $w: A \rightarrow A$ and $w^{\prime}: B \rightarrow B$ such that

$$
w(s(\mathbf{g}) \mathbf{f}) \equiv w\left(1_{(\mathscr{C}, \mathscr{C})}\right) \quad \text { and } \quad w^{\prime}\left(s^{\prime}(\mathbf{f}) \mathbf{g}\right) \equiv w^{\prime}\left(1_{(\mathscr{Y}, \mathscr{V})}\right)
$$

Take any $b \in B$. By (A3) there exists a $b_{0}>b$ such that

$$
q_{b b_{0}}^{-1} \mathscr{V}_{b}>\mathrm{st}^{2} \mathscr{V}_{b_{0}} \text {. }
$$

Let $\phi=q_{b_{0} w^{\prime}\left(b_{0}\right)} q_{w^{\prime}\left(b_{0}\right) s^{\prime} w^{\prime}\left(b_{0}\right)} f_{s^{\prime} w^{\prime}\left(b_{0}\right)}: X_{f s^{\prime} w^{\prime}\left(b_{0}\right)} \rightarrow Y_{b_{0}}$. By the second formula in (3) there exists a $b_{1}>w^{\prime}\left(b_{0}\right), g f s^{\prime} w^{\prime}\left(b_{0}\right)$ such that

(5) $\left(\phi g_{f s^{\prime} w^{\prime}\left(b_{0}\right)} q_{g f s^{\prime} w^{\prime}\left(b_{0}\right) b^{\prime}}, q_{b_{0} w^{\prime}\left(b_{0}\right)} q_{w^{\prime}\left(b_{0}\right) b^{\prime}}\right)<$ st $\mathscr{V}_{b_{0}}$ for any $b^{\prime}>b_{1}$.

By (AM) for $\mathbf{f}$ there exists an $a_{0}>f(b), f s^{\prime} w^{\prime}\left(b_{0}\right)$ such that

(6) $\quad\left(q_{b s^{\prime} w^{\prime}\left(b_{0}\right)} f_{s^{\prime} w^{\prime}\left(b_{0}\right)} p_{f s^{\prime} w^{\prime}\left(b_{0}\right) a^{\prime}}, f_{b} p_{f(b) a^{\prime}}\right)<$ st $\mathscr{V}_{b}$ for any $a^{\prime}>a_{0}$

Take any $a^{\prime}>a_{0}$. By (A3) there exists an $a_{1}>a^{\prime}$ such that

$$
p_{a^{\prime} a_{1}}^{-1} \mathscr{U}_{a^{\prime}}>\mathbf{s t}^{2} \mathscr{U}_{a_{1}} \text {. }
$$


Let $\psi=p_{a_{1} w\left(a_{1}\right)} p_{w\left(a_{1}\right) s w\left(a_{1}\right)} g_{s w\left(a_{1}\right)}: Y_{g s w\left(a_{1}\right)} \rightarrow X_{a_{1}}$. By (3) there exists an $a_{2}>$ $w\left(a_{1}\right), f g s w\left(a_{1}\right)$ such that

(8) $\quad\left(\psi f_{g s w\left(a_{1}\right)} p_{f g s w\left(a_{1}\right) a^{\prime \prime}}, p_{a_{1} w\left(a_{1}\right)} p_{w\left(a_{1}\right) a^{\prime \prime}}\right)<\mathrm{st} \mathscr{U}_{a_{1}}$ for any $a^{\prime \prime}>a_{2}$.

By (AM) for $\mathrm{g}$ there exists a $b_{2}>b_{1}, g s w\left(a_{1}\right)$ such that

(9) $\left(g_{f s^{\prime} w^{\prime}\left(b_{0}\right)} q_{g f s^{\prime} w^{\prime}\left(b_{0}\right) b^{\prime}}, p_{f s^{\prime} w^{\prime}\left(b_{0}\right) s w\left(a_{1}\right)} g_{s w\left(a_{1}\right)} q_{g s w\left(a_{1}\right) b^{\prime}}\right)<s t \mathscr{V}_{f s^{\prime} w^{\prime}\left(b_{0}\right)}$

for any $b^{\prime}>b_{2}$.

Take any $b^{\prime}>b_{2}$. By (AM) for $\mathbf{f}$ there exists an $a_{3}>a_{2}, f\left(b^{\prime}\right)$ such that

$\left(f_{g s w\left(a_{1}\right)} p_{f g s w\left(a_{1}\right) a_{3}}, q_{g s w\left(a_{1}\right) b^{\prime}} f_{b^{\prime}} p_{f\left(b^{\prime}\right) a_{3}}\right)<\operatorname{st} \mathscr{V}_{g s w\left(a_{1}\right)}$.

Let $\alpha=p_{a^{\prime} s w\left(a_{1}\right)} g_{s w\left(a_{1}\right)} q_{g s w\left(a_{1}\right) b_{2}}: Y_{b_{2}} \rightarrow X_{a^{\prime}}$ and $\beta=f_{b^{\prime}} p_{f\left(b^{\prime}\right) a_{3}}: X_{a_{3}} \rightarrow Y_{b^{\prime}}$. Since $\mathbf{f}$ is uniform, $w^{\prime}, s^{\prime}$ are refinement functions, and $\left(p_{f s^{\prime} w^{\prime}\left(b_{0}\right) a^{\prime}} p_{a^{\prime} s w\left(a_{1}\right)}\right.$, $\left.p_{f s^{\prime} w^{\prime}\left(b_{0}\right), s w\left(a_{1}\right)}\right)<\mathscr{U}_{f s^{\prime} w^{\prime}\left(b_{0}\right)}$ by $(\mathrm{A} 1)$,

$$
\left(\phi p_{f s^{\prime} w^{\prime}\left(b_{0}\right) a^{\prime}} \alpha, \phi p_{f s^{\prime} w^{\prime}\left(b_{0}\right) s w\left(a_{1}\right)} g_{s w\left(a_{1}\right)} q_{g s w\left(a_{1}\right) b_{2}}\right)<\mathscr{V}_{b_{0}} .
$$

Since $\mathbf{f}$ is uniform and $s^{\prime}, w^{\prime}$ are refinement functions, by (9)

$$
\left(\phi p_{f s^{\prime} w^{\prime}\left(b_{0}\right) s w\left(a_{1}\right)} g_{s w\left(a_{1}\right)} q_{g s w\left(a_{1}\right) b_{2}}, \phi g_{f s^{\prime} w^{\prime}\left(b_{0}\right)} q_{g f s^{\prime} w^{\prime}\left(b_{0}\right) b_{2}}\right)<\mathscr{V}_{b_{0}} .
$$

Let $\lambda=q_{b_{0} s^{\prime} w^{\prime}\left(b_{0}\right)} f_{s^{\prime} w^{\prime}\left(b_{0}\right)} p_{f s^{\prime} w^{\prime}\left(b_{0}\right) a^{\prime}}: X_{a^{\prime}} \rightarrow Y_{b_{0}}$. By (A1) for $(\mathscr{Y}, \mathscr{V})$

$$
\begin{gathered}
\left(\phi p_{f s^{\prime} w^{\prime}\left(b_{0}\right) a^{\prime}} \alpha, \lambda \alpha\right)<\mathscr{V}_{b_{0}}, \\
\left(q_{b_{0} w^{\prime}\left(b_{0}\right)} q_{w^{\prime}\left(b_{0}\right) b_{2}}, \quad q_{b_{0} b_{2}}\right)<\mathscr{V}_{b_{0}} .
\end{gathered}
$$

By (14), (5), (12), (11), and (13),

$$
\left(q_{b_{0} b_{2}}, \lambda \alpha\right)<\mathbf{s t}^{2} \mathscr{V}_{b_{0}}
$$

By (4) and (15),

$$
\left(q_{b b_{0}} q_{b_{0} b_{2}}, q_{b b_{0}} \lambda \alpha\right)<\mathscr{V}_{b}
$$

By (A1),

$$
\begin{gathered}
\left(q_{b b_{0}} q_{b_{0} b_{2}}, q_{b b_{2}}\right)<\mathscr{V}_{b}, \\
\left(q_{b b_{0}} \lambda \alpha, q_{b s^{\prime} w^{\prime}\left(b_{0}\right)} f_{s^{\prime} w^{\prime}\left(b_{0}\right)} p_{f s^{\prime} w^{\prime}\left(b_{0}\right) a^{\prime}} \alpha\right)<\mathscr{V}_{b} .
\end{gathered}
$$

By (6),

$$
\left(q_{b s^{\prime} w^{\prime}\left(b_{0}\right)} f_{s^{\prime} w^{\prime}\left(b_{0}\right)} p_{f s^{\prime} w^{\prime}\left(b_{0}\right) a^{\prime}} \alpha, f_{b} p_{f(b) a^{\prime}} \alpha\right)<s t \mathscr{V}_{b} .
$$

By (17), (16), (18), and (19),

$$
\left(q_{b b_{2}}, f_{b} p_{f(b) a^{\prime}} \alpha\right)<\mathrm{st}^{2} \mathscr{V}_{b}
$$

Since $s, w$ are refinement functions and $\mathbf{g}$ is uniform, by (10) and (A1) for $(\mathscr{Y}, \mathscr{V})$

$$
\begin{gathered}
\left(\psi f_{g s w\left(a_{1}\right)} p_{f g s w\left(a_{1}\right) a_{3}}, \psi \psi q_{g s w\left(a_{1}\right) b^{\prime}} f_{b^{\prime}} p_{f\left(b^{\prime}\right) a_{3}}\right)<\mathscr{U}_{a_{1}}, \\
\left(\psi \omega, \quad \psi q_{g s w\left(a_{1}\right) b^{\prime}} f_{b^{\prime}} p_{f\left(b^{\prime}\right) a_{3}}\right)<\mathscr{U}_{a_{1}},
\end{gathered}
$$

where $\omega=q_{g s w\left(a_{1}\right) b_{2}} q_{b_{2} b^{\prime}} f_{b^{\prime}} p_{f\left(b^{\prime}\right) a_{3}}: X_{a_{3}} \rightarrow Y_{g s w\left(a_{1}\right)}$. By (A1) for $(\mathscr{X}, \mathscr{U})$

$$
\left(\psi \omega, p_{a_{1} s w\left(a_{1}\right)} g_{s w\left(a_{1}\right)} \omega\right)<\mathscr{U}_{a_{1}} .
$$


Since $\left(p_{a_{1} w\left(a_{1}\right)} p_{w\left(a_{1}\right) a_{3}}, p_{a_{1} a_{3}}\right)<\mathscr{U}_{a_{1}}$, by (8) for $a^{\prime \prime}=a_{3},(21),(22)$, and (23)

$$
\left(p_{a_{1} a_{3}}, p_{a_{1} s w\left(a_{1}\right)} g_{s w\left(a_{1}\right)} \omega\right)<\mathrm{st}^{2} \mathscr{U}_{a_{1}} \text {. }
$$

By (7) and (24),

$$
\left(p_{a^{\prime} a_{1}} p_{a_{1} a_{3}}, p_{a^{\prime} a_{1}} p_{a_{1} s w\left(a_{1}\right)} g_{s w\left(a_{1}\right)} \omega\right)<\mathscr{U}_{a}^{\prime} .
$$

By (A1) for $(\mathscr{X}, \mathscr{U})$,

$$
\left(p_{a^{\prime} a_{1}} p_{a_{1} s w\left(a_{1}\right)} g_{s w\left(a_{1}\right)} \omega, \alpha q_{b_{2} b^{\prime}} \beta\right)<\mathscr{U}_{a}^{\prime} .
$$

Since $\left(p_{a^{\prime} a_{1}} p_{a_{1} a_{3}}, p_{a^{\prime} a_{3}}\right)<\mathscr{U}_{a}^{\prime}$ by $(\mathrm{A} 1)$, by (25) and (26)

$$
\left(p_{a^{\prime} a_{3}}, \alpha q_{b_{2} b^{\prime}} \beta\right)<\text { st } \mathscr{U}_{a}^{\prime} \text {. }
$$

Inequalities (20) and (27) mean that $f$ satisfies (API).

Let $f: X \rightarrow Y$ be a map. Let $\mathbf{p}: X \rightarrow(\mathscr{X}, \mathscr{U}), \mathbf{p}^{\prime}: X \rightarrow(\mathscr{X}, \mathscr{U})^{\prime}$, $\mathbf{q}: Y \rightarrow(\mathscr{Y}, \mathscr{V})$, and $\mathbf{q}^{\prime}: Y \rightarrow(\mathscr{Y}, \mathscr{V})^{\prime}$ be approximate APOL-resolutions, respectively. Then we have the following lemma.

(8.4) Lemma. Let ( $\mathbf{p}, \mathbf{q}, \mathbf{f})$ and $\left(\mathbf{p}^{\prime}, \mathbf{q}^{\prime}, \mathbf{f}^{\prime}\right)$ be approximate APOL-resolutions of $f$. Then $\mathbf{f}$ is an approximate pseudo-isomorphism if and only if $\mathbf{f}^{\prime}$ is.

Proof. By (1.15) there exist approximate resolutions $\left(\mathbf{p}, \mathbf{p}^{\prime}, \mathbf{u}\right),\left(\mathbf{q}, \mathbf{q}^{\prime}, \mathbf{v}\right)$, and $\left(\mathbf{p}, \mathbf{q}^{\prime}, \mathbf{h}\right)$ of $1_{X}, 1_{Y}$, and $\mathbf{f}$, respectively. Thus by $(1.17)\left[\mathbf{f}^{\prime}\right][\mathbf{u}]=[\mathbf{h}]=$ $[\mathbf{v}]\left[\mathbf{f}^{\prime}\right]$. By (1.18) [u] and [v] are isomorphisms. So if [f] is an approximate pseudo-isomorphism, then $\left[\mathbf{f}^{\prime}\right]=[\mathbf{v}][\mathbf{f}][\mathbf{u}]^{-1}$ is also an approximate pseudoisomorphism by (8.2) and (8.3). The converse follows in the same way.

By (8.4) we may say a map $f: X \rightarrow Y$ is an approximate pseudo-isomorphism provided some/any of its approximate APOL-resolutions is an approximate pseudo-isomorphism.

(8.5) Lemma. Let $(\mathscr{X}, \mathscr{U}),(\mathscr{Y}, \mathscr{V})$ and $\mathbf{f}:(\mathscr{X}, \mathscr{U}) \rightarrow(\mathscr{Y}, \mathscr{V})$ be approximate inverse systems and an approximate system map in the sense of [W1], respectively. Then $\mathbf{f}$ satisfies (API) if and only if it satisfies the following condition:

(API1)

$$
\begin{aligned}
& (\forall b \in B)\left(\forall a^{\prime}>f(b)\right)\left(\exists b^{\prime}>b\right)\left(\exists g: Y_{b^{\prime}} \rightarrow X_{a^{\prime}}\right) \\
& \left(f_{b} p_{f(b) a^{\prime}} g, q_{b b^{\prime}}\right)<\mathrm{st} \mathscr{V}_{b} \\
& \left(\forall b^{\prime \prime}>b_{1}\right)\left(\exists a^{\prime \prime}>a\right)\left(\exists h: X_{a^{\prime \prime}} \rightarrow Y_{b^{\prime \prime}}\right) \\
& \left(g q_{b^{\prime} b^{\prime \prime}} h, p_{a^{\prime} a^{\prime \prime}}\right)<\mathscr{U}_{a^{\prime}} .
\end{aligned}
$$

Proof. Note that $(\mathscr{X}, \mathscr{U}),(\mathscr{Y}, \mathscr{V})$ are approximate commutative inverse systems and $\mathbf{f}$ is uniform by (AM2) of [W1].

We show that (API) implies (API1). Take any $b \in B$ and $a \in A$ with $a>f(b)$. By (A3) there exists a $b_{0}>b$ such that

$$
q_{b b_{0}}^{-1} \mathscr{V}_{b}>\mathrm{st}^{2} \mathscr{V}_{b_{0}} \text {. }
$$

By (AM2) of [W1] there exists an $a_{1}>f(b), f\left(b_{0}\right)$ such that

$$
\left(f_{b} p_{f(b) a_{1}}, q_{b b_{0}} f_{b_{0}} p_{f\left(b_{0}\right) a_{1}}\right)<\mathscr{V}_{b} .
$$

By the assumption, there exists an $a_{0}>f\left(b_{0}\right)$ satisfying (API) for $b_{0}$. By (A3) there exists an $a_{2}>a_{0}, a_{1}, a$ such that

$$
p_{a a_{2}}^{-1} \mathscr{U}_{a}>\text { st } \mathscr{U}_{a_{2}} .
$$


By the choice of $a_{0}$, there exist a $b_{1}>b_{0}$ and a map $g: Y_{b_{1}} \rightarrow X_{a_{2}}$ such that

$$
\left(f_{b_{0}} p_{f\left(b_{0}\right) a_{2}} g, q_{b_{0} b_{1}}\right)<\mathbf{s t}^{2} \mathscr{V}_{b_{0}},
$$

and satisfying (API) for $a_{0}, b_{1}$, and $g$. Take any $b^{\prime}>b_{1}$. By the choices of $a_{0}, b_{1}$, and $g$, there exists an $a_{3}>a_{2}$ and a map $h: X_{a_{3}} \rightarrow Y_{b^{\prime}}$ such that

$$
\left(g q_{b_{1} b^{\prime}} h, p_{a_{2} a_{3}}\right)<\mathrm{st} \mathscr{U}_{a_{2}} .
$$

Let $\alpha=p_{a a_{2}} g: Y_{b_{1}} \rightarrow X_{a}$. Since $(\mathscr{Y}, \mathscr{V})$ is commutative, by (1) and (4) we have

$$
\left(q_{b b_{0}} f_{b_{0}} p_{f\left(b_{0}\right) a_{2}} g, q_{b b_{1}}\right)<\mathscr{V}_{b} .
$$

Since $(\mathscr{X}, \mathscr{U})$ is commutative, by $(2)$

$$
\left(f_{b} p_{f(b) a} \alpha, q_{b b_{0}} f_{b_{0}} p_{f\left(b_{0}\right) a_{2}} g\right)<\mathscr{V}_{b} .
$$

By (6) and (7) we have

$$
\left(f_{b} p_{f(b) a} \alpha, q_{b b_{1}}\right)<\mathrm{st} \mathscr{V}_{b} .
$$

Since $(\mathscr{X}, \mathscr{U})$ is commutative, by $(3)$ and (5) we have

$$
\left(\alpha q_{b_{1} b^{\prime}} h, p_{a a_{3}}\right)<\mathscr{U}_{a} \text {. }
$$

Thus (8) and (9) mean that $f$ satisfies (API1).

Finally, we show that (API1) implies (API). This is done by taking $a_{0}=f(b)$ for any $b \in B$ in (API).

Let $f: X \rightarrow Y$ be a map. Let $\mathscr{U} \in \operatorname{Cov}(X)$. We say that $f$ is a $\mathscr{U}$-map provided for each $y \in Y, f^{-1}(y) \subset U$ for some $U \in \mathscr{U}$. We say that $f$ is a refinable map provided $f$ is onto and it satisfies the following condition: For any $\mathscr{U} \in \operatorname{Cov}(X)$ and $\mathscr{V} \in \operatorname{Cov}(Y)$ there exists a map $g: X \rightarrow Y$ such that $g$ is a $\mathscr{U}$-map and $(f, g)<\mathscr{V}$.

Clearly, the above definition of refinability for a map between compact metric spaces is equivalent to the original one of Ford and Rogers [FR].

(8.6) Theorem. If $f: X \rightarrow Y$ is a refinable map between compact Hausdorff spaces, then $f$ is an approximate pseudo-isomorphism.

In Chapters 21 and 27 of [W] we investigated property (API1) for approximate system maps in the sense of [W1]. Also we showed that any approximate resolution in the sense of [W1] of a refinable map between compact Hausdorff spaces satisfies (API1). Thus by (8.5) we have (8.6). Koyama $[\mathrm{K}]$ generalized (8.6) to locally compact paracompact spaces in the commutative case. So (8.5) and his proof also yield a proof of (8.6). Since his proof has been published, we omit our proof.

(8.7) Theorem. Let $\mathbf{f}:(\mathscr{X}, \mathscr{U}) \rightarrow(\mathscr{Y}, \mathscr{V})$ be an approximate pseudo-isomorphism. If $(\mathscr{Z}, \mathscr{U})$ satisfies $(\mathrm{AC})$, then so does $(\mathscr{Y}, \mathscr{V})$.

Proof. Take any $b \in B$ and a $\mathscr{V} \in \operatorname{Cov}\left(Y_{b}\right)$ such that st $\mathscr{V}<\mathscr{V}_{b}$. There exists a $b_{1}>b$ such that

$$
\begin{gathered}
q_{b b^{\prime}}^{-1}(\mathscr{V})>\mathrm{st}^{3} \mathscr{V}_{b^{\prime}} \text { for any } b^{\prime}>b_{1}, \\
\left(q_{b b^{\prime}} q_{b^{\prime} b^{\prime \prime}}, q_{b b^{\prime \prime}}\right)<\mathscr{V} \text { for any } b^{\prime \prime}>b^{\prime}>b_{1} .
\end{gathered}
$$


There exists an $a_{2}>f\left(b_{1}\right)$ such that

$$
p_{f\left(b_{1}\right) a^{\prime}}^{-1}\left(f_{b_{1}}^{-1} \mathscr{V}_{b_{1}}\right)>\mathscr{U}_{a^{\prime}} \text { for any } a^{\prime}>a_{2},
$$

$$
\left(p_{f\left(b_{1}\right) a^{\prime}} p_{a^{\prime} a^{\prime \prime}}, p_{f\left(b_{1}\right) a^{\prime \prime}}\right)<f_{b_{1}}^{-1} \mathscr{V}_{b_{1}} \text { for } a^{\prime \prime}>a^{\prime}>a_{2} \text {. }
$$

By the assumption, there exists an $a_{3}>a_{2}$ satisfying condition (AC) for $(\mathscr{X}, \mathscr{U})$ and $a_{2}$. Since $\mathbf{f}$ is an approximate pseudo-isomorphism, applying Lemma (8.5), there exist a $b_{2}>b_{1}$ and a map $g: Y_{b_{2}} \rightarrow X_{a_{3}}$ and for each $b_{3}>b_{2}$ there exist an $a_{4}>a_{3}$ and a map $h: X_{a_{4}} \rightarrow Y_{b_{3}}$ such that

$$
\begin{gathered}
\left(f_{b_{1}} p_{f\left(b_{1}\right) a_{3}} g, q_{b_{1} b_{2}}\right)<\mathrm{st}^{2} \mathscr{V}_{b_{1}}, \\
\left(g q_{b_{2} b_{3}} h, p_{a_{3} a_{4}}\right)<\mathrm{st} \mathscr{U}_{a_{3}} .
\end{gathered}
$$

Take any map $\alpha: Y_{b_{3}} \rightarrow Y_{b_{2}}$. We may consider $\alpha^{\prime}=g \alpha h: X_{a_{4}} \rightarrow X_{a_{3}}$. By the choice of $a_{3}$, there exist a point $x_{0} \in X_{a_{4}}$ and a $U_{0} \in \mathscr{U}_{a_{2}}$ such that

$$
p_{a_{2} a_{3}} p_{a_{3} a_{4}}\left(x_{0}\right), p_{a_{2} a_{3}} \alpha^{\prime}\left(x_{0}\right) \in U_{0} \text {. }
$$

By (3) and (7), there exists a $V_{0} \in \mathscr{V}_{b_{1}}$ such that

$$
f_{b_{1}} p_{f\left(b_{1}\right) a_{2}} p_{a_{2} a_{3}} p_{a_{3} a_{4}}\left(x_{0}\right), f_{b_{1}} p_{f\left(b_{1}\right) a_{2}} p_{a_{2} a_{3}} \alpha^{\prime}\left(x_{0}\right) \in V_{0}
$$

By (4),

$$
\left(f_{b_{1}} p_{f\left(b_{1}\right) a_{2}} p_{a_{2} a_{3}}, f_{b_{1}} p_{f\left(b_{1}\right) a_{3}}\right)<\mathscr{V}_{b_{1}} \text {. }
$$

Thus, by (9), there exist $V_{1}, V_{2} \in \mathscr{V}_{b_{1}}$ such that

$$
\begin{gathered}
f_{b_{1}} p_{f\left(b_{1}\right) a_{2}} p_{a_{2} a_{3}} p_{a_{3} a_{4}}\left(x_{0}\right), f_{b_{1}} p_{f\left(b_{1}\right) a_{3}} p_{a_{3} a_{4}}\left(x_{0}\right) \in V_{1}, \\
f_{b_{1}} p_{f\left(b_{1}\right) a_{2}} p_{a_{2} a_{3}} \alpha^{\prime}\left(x_{0}\right), f_{b_{1}} p_{f\left(b_{1}\right) a_{3}} \alpha^{\prime}\left(x_{0}\right) \in V_{2} .
\end{gathered}
$$

By (3) and (6),

$$
\left(f_{b_{1}} p_{f\left(b_{1}\right) a_{3}} g q_{b_{2} b_{3}} h, f_{b_{1}} p_{f\left(b_{1}\right) a_{3}} p_{a_{3} a_{4}}\right)<\mathscr{V}_{b_{1}}
$$

By (5),

$$
\left(f_{b_{1}} p_{f\left(b_{1}\right) a_{3}} g \alpha h, q_{b_{1} b_{2}} \alpha h\right)<\mathbf{s t}^{2} \mathscr{V}_{b_{1}},
$$

By (12), (13), and (14), there exist $V_{3} \in \mathscr{V}_{b_{1}}, V_{4}, V_{5} \in \mathrm{st}^{2} \mathscr{V}_{b_{1}}$ such that

$$
\begin{gathered}
f_{b_{1}} p_{f\left(b_{1}\right) a_{3}} g q_{b_{2} b_{3}} h\left(x_{0}\right), f_{b_{1}} p_{f\left(b_{1}\right) a_{3}} p_{a_{3} a_{4}}\left(x_{0}\right) \in V_{3}, \\
f_{b_{1}} p_{f\left(b_{1}\right) a_{3}} \alpha^{\prime}\left(x_{0}\right), q_{b_{1} b_{2}} \alpha h\left(x_{0}\right) \in V_{4}, \\
f_{b_{1}} p_{f\left(b_{1}\right) a_{3}} g q_{b_{2} b_{3}} h\left(x_{0}\right), q_{b_{1} b_{2}} q_{b_{2} b_{3}} h\left(x_{0}\right) \in V_{5} .
\end{gathered}
$$

By (16), (11), (8), (10), (15), and (17), there exists a $V_{6} \in \mathbf{s t}^{3} \mathscr{V}_{b_{1}}$ such that

$$
q_{b_{1} b_{2}} \alpha h\left(x_{0}\right), q_{b_{1} b_{2}} q_{b_{2} b_{3}} h\left(x_{0}\right) \in V_{6}
$$

By (1) and (18), there exists a $V_{7} \in \mathscr{V}$ such that

$$
q_{b b_{1}} q_{b_{1} b_{2}} \alpha h\left(x_{0}\right), q_{b b_{1}} q_{b_{1} b_{2}} q_{b_{2} b_{3}} h\left(x_{0}\right) \in V_{7} \text {. }
$$


By (2), there exist $V_{8}, V_{9} \in \mathscr{V}_{b}$ such that

$$
\begin{gathered}
q_{b b_{1}} q_{b_{1} b_{2}} \alpha h\left(x_{0}\right), q_{b b_{2}} \alpha h\left(x_{0}\right) \in V_{8}, \\
q_{b b_{1}} q_{b_{1} b_{2}} q_{b_{2} b_{3}} h\left(x_{0}\right), q_{b b_{2}} q_{b_{2} b_{3}} h\left(x_{0}\right) \in V_{9} .
\end{gathered}
$$

By (20), (19), (21), and the choice of $\mathscr{V}$, there exists a $V \in \mathscr{V}_{b}$ such that

$$
q_{b b_{2}} \alpha h\left(x_{0}\right), q_{b b_{2}} q_{b_{2} b_{3}} h\left(x_{0}\right) \in \operatorname{st}\left(V_{7}, \mathscr{V}_{b}\right) \subset V .
$$

Therefore, we put $y_{0}=h\left(x_{0}\right)$; then (22) means that $b_{2}$ satisfies condition (AC) for $b$. Hence, $(\mathscr{Y}, \mathscr{V})$ satisfies $(\mathrm{AC})$.

(8.8) Corollary. Let $f: X \rightarrow Y$ be a refinable map between compact Hausdorff spaces. If $X$ is cosmic, then so is $Y$.

We consider the question: Does a refinable map preserve the fixed point property? The next example gives a negative answer to this question.

(8.9) Example. There exists a refinable map $f: X \rightarrow Y$ between compacta such that $X$ has the fixed point property, but $Y$ does not have it.

Proof. We shall use the same notation as in Example (5.19). Let $X=\overline{b_{0} a_{0}} \cup$ $\overline{a_{0} a_{1}} \cup \overline{a_{1} b_{1}} \cup \bigcup_{i=1}^{\infty} \overline{b_{i} b_{i+1}}$ be a continuum in $R^{2}$. Let $Y=\overline{c_{0} a_{0}} \cup \overline{a_{0} a_{1}} \cup \overline{a_{1} c_{1}} \cup \overline{c_{0} c_{1}}$ be a continuum in $R^{2}$. $X$ and $Y$ are the Warsaw circle and a circle, respectively.

We define a map $\pi: R^{2} \rightarrow R^{2}$ as follows:

$$
\pi((x, y))= \begin{cases}(x, 0) & \text { if } y \geq 0 \\ (x, y) & \text { if } y \leq 0\end{cases}
$$

Clearly $\pi$ is continuous and $\pi(X)=Y$. Let $f=\pi \mid X: X \rightarrow Y$. We show that Claim. $f$ is a refinable map.

Take any $\varepsilon>0$ and then there is an integer $n$ with $\frac{1}{n}<\varepsilon$. We put $b_{1}^{\prime}=$ $\left(1-\frac{1}{2 n+1}, 1\right) \in R^{2}$. We put

$$
Z_{n+1}=\overline{b_{2 n+1} c_{0}} \cup \overline{c_{0} a_{0}} \cup \overline{a_{0} a_{1}} \cup \overline{a_{1} c_{1}} \cup \overline{c_{1} b_{1}^{\prime}} \cup \overline{b_{1}^{\prime} b_{2}} \cup \bigcup_{j=2}^{2 n} \overline{b_{j} b_{j+1}} \subset R^{2} .
$$

We define a map $r_{2 n+1}: X \rightarrow Z_{2 n+1}$ as follows:

$$
r_{2 n+1}((x, y))= \begin{cases}\left(\frac{y}{2 n+1}, y\right) & \text { for } 0 \leq x \leq \frac{1}{2 n+1}, 0 \leq y \leq 1, \\ \left(\frac{2 n-1}{2 n+1} y+\frac{1}{2}, y\right) & \text { for } \frac{1}{2} \leq x<1,0 \leq y \leq 1, \\ \left(1-\frac{y}{2 n+1}, y\right) & \text { for } x=1,0 \leq y \leq 1, \\ (x, y) & \text { otherwise. }\end{cases}
$$

Finally, we put $f_{\varepsilon}=\pi r_{2 n+1}: X \rightarrow Z_{2 n+1} \rightarrow Y$. By definition, $d\left(f, f_{\varepsilon}\right)<\varepsilon$ and $f_{\varepsilon}$ is an $\varepsilon$-mapping. Thus $f$ is a refinable map.

It is well known that $X$ has the fixed point property (Theorem 13 of [B]), but $Y$ does not. Hence, the refinable map $f$ does not preserve the fixed point property.

\section{HYPERSPACES AND THE FIXED POINT PROPERTY}

In 1952, Knaster raised the question of when does the hyperspace $C(X)$ have the fixed point property (see Chapter VII of $[\mathrm{N}]$ ). There have been a number 
of positive and negative results given for this question in the compact metric case. Here we extend some of these results to the compact Hausdorff case as an application of the theorems obtained in $\S 5$.

Let $X$ be a compact Hausdorff space. Let $2^{X}$ be the set of all nonempty closed subsets of $X$. Let $C(X)$ be the set of all nonempty closed connected subsets of $X$.

Let $U_{1}, U_{2}, \ldots, U_{n}$ be open subsets of $X$. Let $\left\langle U_{1}, U_{2}, \ldots, U_{n}\right\rangle=\{K \in$ $2^{X}: K \subset \bigcup_{i=1}^{n} U_{i}$ and $U_{i} \cap K \neq \varnothing$ for each $\left.i, 1 \leq i \leq n\right\}$. Then, $\mathscr{B}=$ $\left\{\left\langle U_{1}, U_{2}, \ldots, U_{n}\right\rangle: U_{1}, \ldots, U_{n}\right.$ are open subsets of $X$ and $\left.n=1,2, \ldots\right\}$ is a basis of the Vietoris topology of $2^{X}$. Let $C(X)$ be a subspace of $2^{X} \cdot 2^{X}$ and $C(X)$ are hyperspaces of $X$.

Let $\mathscr{U}$ be an open covering of $X$. Let $\langle\mathscr{U}\rangle=\left\{\left\langle U_{1}, U_{2}, \ldots, U_{n}\right\rangle: U_{1}, U_{2}\right.$, $\ldots, U_{n} \in \mathscr{U}$ and $\left.n=1,2, \ldots\right\}$. Morita [Mo] has proved that $\{\langle\mathscr{U}\rangle: \mathscr{U} \in$ $\operatorname{Cov}(X)\}$ is a uniformity of $2^{X}$. By the uniqueness of the uniformity on a compact Hausdorff space, we have

(9.1) Lemma (see (3.1) of [W3]). The uniformities $\{\langle\mathscr{U}\rangle: \mathscr{U} \in \operatorname{Cov}(X)\}$ and $\operatorname{Cov}\left(2^{X}\right)$ are equivalent, that is, for each $\mathscr{W} \in \operatorname{Cov}\left(2^{X}\right)$ there exists a $\mathscr{U} \in$ $\operatorname{Cov}(X)$ such that $\langle\mathscr{U}\rangle<\mathscr{W}$.

Let $f: X \rightarrow Y$ be a map between compact Hausdorff spaces. Then $f$ induces maps $\bar{f}: 2^{X} \rightarrow 2^{Y}$ and $\bar{f}: C(X) \rightarrow C(Y)$.

(9.2) Lemma (see (3.2) of [W3]). Let $\mathscr{U} \in \operatorname{Cov}(X)$ and $\mathscr{V} \in \operatorname{Cov}(Y)$. If $f^{-1 \mathscr{V}}>\mathscr{U}$, then $(\bar{f})^{-1}(\langle\mathscr{V}\rangle)>\langle\mathscr{U}\rangle$ for $\bar{f}: 2^{X} \rightarrow 2^{Y}$. Similarly, this holds for $\bar{f}: C(X) \rightarrow C(Y)$.

(9.3) Lemma (see (3.4) of [W3]). Let $f, g: X \rightarrow Y$ be maps and $\mathscr{V} \in$ $\operatorname{Cov}(Y)$. If $(f, g)<\mathscr{V}$, then $(\bar{f}, \bar{g})<\langle\mathscr{V}\rangle$ for $\bar{f}, \bar{g}: 2^{X} \rightarrow 2^{Y}$. Similarly, this holds for $\bar{f}, \bar{g}: C(X) \rightarrow C(Y)$.

Let $(\mathscr{X}, \mathscr{U})=\left\{X_{a}, \mathscr{U}_{a}, p_{a a^{\prime}}, A\right\}$ be an approximate inverse system of compact Hausdorff spaces $X_{a}$. Thus, we define $2^{(\mathscr{X}, \mathscr{U})}=\left\{2^{X_{a}},\left\langle\mathscr{U}_{a}\right\rangle, \bar{p}_{a a^{\prime}}, A\right\}$ and $C(\mathscr{Z}, \mathscr{U})=\left\{C\left(X_{a}\right),\left\langle\mathscr{U}_{a}\right\rangle \mid C\left(X_{a}\right), \bar{p}_{a a^{\prime}}, A\right\}$.

(9.4) Lemma. If $(\mathscr{X}, \mathscr{U})$ is an approximate inverse system, then so are $2^{(\mathscr{X}, \mathscr{U})}$ and $C(\mathscr{X}, \mathscr{U})$.

Proof. We must show the conditions (A1)-(A3) for $2^{(\mathscr{X}, \mathscr{U})}$.

Since $(\mathscr{X}, \mathscr{U})$ is an approximate inverse system, it satisfies (A1)-(A3). By (A1) for $(\mathscr{X}, \mathscr{U})$, for $a<a^{\prime}<a^{\prime \prime},\left(p_{a a^{\prime}} p_{a^{\prime} a^{\prime \prime}}, p_{a a^{\prime \prime}}\right)<\mathscr{U}_{a}$. Since $\overline{(f g)}=\bar{f} \cdot \bar{g}$ for $f: X \rightarrow Y, g: Y \rightarrow Z$, by Lemma (9.3), $\left(\bar{p}_{a a^{\prime}} \bar{p}_{a^{\prime} a^{\prime \prime}}, \bar{p}_{a a^{\prime \prime}}\right)<\left\langle\mathscr{U}_{a}\right\rangle$. Thus $2^{(\mathscr{Z}, \mathscr{U})}$ satisfies $(\mathrm{A} 1)$.

To show (A2) for $2^{(\mathscr{X}, \mathscr{U})}$, take any $a \in A$ and any $\mathscr{U} \in \operatorname{Cov}\left(2^{X_{a}}\right)$. By Lemma (9.1), there exists a $\mathscr{V} \in \operatorname{Cov}\left(X_{a}\right)$ such that

$$
\langle\mathscr{V}\rangle<\mathscr{U} \text {. }
$$

By (A2) for $(\mathscr{X}, \mathscr{U})$, there exists an $a^{\prime}>a$ such that

$$
\left(p_{a a_{1}} p_{a_{1} a_{2}}, p_{a a_{2}}\right)<\mathscr{V} \text { for any } a_{2}>a_{1}>a^{\prime} \text {. }
$$

By Lemma (9.3), (1), and (2),

$$
\left(\bar{p}_{a a_{1}} \bar{p}_{a_{1} a_{2}}, \bar{p}_{a a_{2}}\right)<\langle\mathscr{V}\rangle<\mathscr{U} \text { for any } a_{2}>a_{1}>a^{\prime} .
$$

Hence, by (3), $2^{(\mathscr{X}, \mathscr{U})}$ satisfies (A2). 
To show (A3) for $2^{(\mathscr{X}, \mathscr{U})}$, take any $a \in A$ and any $\mathscr{U} \in \operatorname{Cov}\left(2^{X_{a}}\right)$. By Lemma (9.1), there exists a $\mathscr{V} \in \operatorname{Cov}\left(X_{a}\right)$ satisfying (1). By (A3) for $(\mathscr{X}, \mathscr{U})$, there exists an $a^{\prime}>a$ such that

$$
\mathscr{U}_{a^{\prime \prime}}<p_{a a^{\prime \prime}}^{-1} \mathscr{V} \text { for any } a^{\prime \prime}>a^{\prime} .
$$

By Lemma (9.2), (4) and (1),

$$
\left\langle\mathscr{U}_{a^{\prime \prime}}\right\rangle<\left(\bar{p}_{a a^{\prime \prime}}\right)^{-1}(\langle\mathscr{V}\rangle)<\left(\bar{p}_{a a^{\prime \prime}}\right)^{-1}(\mathscr{U}) \text { for any } a^{\prime \prime}>a^{\prime} .
$$

Hence, by $(5), 2^{(\mathscr{X}, \mathscr{U})}$ satisfies (A3). Therefore, $2^{(\mathscr{X}, \mathscr{U})}$ is an approximate inverse system. Similarly, we can show that $C(\mathscr{Z}, \mathscr{U})$ is an approximate inverse system.

Let $\mathbf{p}=\left\{p_{a}: a \in A\right\}: X \rightarrow(\mathscr{X}, \mathscr{U})$ be an approximate map. p induces the collection of maps $\overline{\mathbf{p}}=\left\{\bar{p}_{a}: a \in A\right\}: 2^{X} \rightarrow 2^{(\mathscr{L}, \mathscr{U})}$ and also $\overline{\mathbf{p}}=\left\{\bar{p}_{a}: a \in A\right\}:$ $C(X) \rightarrow C(\mathscr{X}, \mathscr{U})$.

(9.5) Lemma. If $\mathbf{p}: X \rightarrow(\mathscr{Z}, \mathscr{U})$ is an approximate map, then so are $\overline{\mathbf{p}}$ : $2^{X} \rightarrow 2^{(\mathscr{L}, \mathscr{U})}$ and $\overline{\mathbf{p}}: C(X) \rightarrow C(\mathscr{X}, \mathscr{U})$.

Proof. We show that condition (AS) of (1.7) is satisfied. To do so, take any $a \in A$ and any $\mathscr{U} \in \operatorname{Cov}\left(2^{X_{a}}\right)$. By Lemma (9.1), there exists a $\mathscr{V} \in \operatorname{Cov}\left(X_{a}\right)$ such that

$$
\langle\mathscr{V}\rangle<\mathscr{U} .
$$

Since $\mathbf{p}: X \rightarrow(\mathscr{X}, \mathscr{U})$ is an approximate map, it satisfies condition (AS). Thus, there exists an $a^{\prime}>a$ such that

$$
\left(p_{a a^{\prime \prime}} p_{a^{\prime \prime}}, p_{a}\right)<\mathscr{V} \text { for any } a^{\prime \prime}>a^{\prime} .
$$

By Lemma (9.3), (2) and (1),

$$
\left(\bar{p}_{a a^{\prime \prime}} \bar{p}_{a^{\prime \prime}}, \bar{p}_{a}\right)<\langle\mathscr{V}\rangle<\mathscr{U} \text { for any } a^{\prime \prime}>a^{\prime} .
$$

By (3), $\overline{\mathbf{p}}: 2^{X} \rightarrow 2^{(\mathscr{X}, \mathscr{U})}$ satisfies (AS). Similarly, $\overline{\mathbf{p}}: C(X) \rightarrow C(\mathscr{X}, \mathscr{U})$ satisfies (AS).

(9.6) Lemma. Let $f: X \rightarrow Y$ be a map.

(i) If $\mathscr{V} \in \operatorname{Cov}(Y)$, then $(\bar{f})^{-1}(\langle\mathscr{V}\rangle)<\left\langle f^{-1 \mathscr{V}\rangle}\right.$ for $\bar{f}: 2^{X} \rightarrow 2^{Y}$ and $\bar{f}: C(X) \rightarrow C(Y)$.

(ii) Let $\mathscr{U} \in \operatorname{Cov}(X)$ and $\mathscr{V} \in \operatorname{Cov}(Y)$. If $f^{-1} \mathscr{V}<\mathscr{U}$, then $(\bar{f})^{-1}(\langle\mathscr{V}\rangle)$ $<\langle\mathscr{U}\rangle$ for $\bar{f}: 2^{X} \rightarrow 2^{Y}$ and $\bar{f}: C(X) \rightarrow C(Y)$.

Proof. We show (i) and (ii) for only $\bar{f}: 2^{X} \rightarrow 2^{Y}$. We first show (i).

Take any $K_{1}, \ldots, V_{n} \in \mathscr{V}$ and any $K \in(\bar{f})^{-1}\left(\left\langle V_{1}, V_{2}, \ldots, V_{m}\right\rangle\right)$. Since $\bar{f}(K)=f(K), f(K) \in\left\langle V_{1}, V_{2}, \ldots, V_{n}\right\rangle$. Thus,

$$
\begin{gathered}
f(K) \subset V_{1} \cup V_{2} \cup \cdots \cup V_{n}, \\
f(K) \cap V_{j} \neq \varnothing \quad \text { for any } j, 1 \leq j \leq n .
\end{gathered}
$$

By (1),

$$
K \subset f^{-1} V_{1} \cup f^{-1} V_{2} \cup \cdots \cup f^{-1} V_{n} .
$$


By (2),

$$
K \cap f^{-1} V_{j} \neq \varnothing \quad \text { for any } j, 1 \leq j \leq n .
$$

Equations (3) and (4) mean that

$$
K \in\left\langle f^{-1} V_{1}, f^{-1} V_{2}, \ldots, f^{-1} V_{n}\right\rangle
$$

Thus, by (5), we have

$$
(\bar{f})^{-1}\left(\left\langle V_{1}, V_{2}, \ldots, V_{n}\right\rangle\right) \subset\left\langle f^{-1} V_{1}, f^{-1} V_{2}, \ldots, f^{-1} V_{n}\right\rangle .
$$

Since $\left\langle f^{-1} V_{1}, f^{-1} V_{2}, \ldots, f^{-1} V_{n}\right\rangle \in\left\langle f^{-1} \mathscr{V}\right\rangle,(6)$ means that $(\bar{f})^{-1}(\langle\mathscr{V}\rangle)<$ $\left\langle f^{-1 \mathscr{V}\rangle}\right\rangle$. Thus we have (i).

We now show (ii). Since $f^{-1 \mathscr{V}}<\mathscr{U}$,

$$
\left\langle f^{-1 \mathscr{V}\rangle}<\langle\mathscr{U}\rangle\right. \text {. }
$$

By (i) and (7), $(\bar{f})^{-1}(\langle\mathscr{V}\rangle)<\left\langle f^{-1} \mathscr{V}\right\rangle\left\langle\langle\mathscr{U}\rangle\right.$. Hence $(\bar{f})^{-1}(\langle\mathscr{V}\rangle)<\langle\mathscr{U}\rangle$. Hence we have (ii). Similarly, (i) and (ii) hold for $\bar{f}: C(X) \rightarrow C(Y)$.

(9.7) Lemma. Let $f: X \rightarrow Y$ be a map. Let $S \subset X$ and $T \subset Y$ be subspaces. Let $\mathscr{U} \in \operatorname{Cov}(X)$ and $\mathscr{V} \in \operatorname{Cov}(Y)$. If $f(S) \subset \operatorname{st}(T, \mathscr{V})$ and $f^{-1} \mathscr{V}>$ st $\mathscr{U}$, then $f(\overline{\operatorname{st}(S, \mathrm{st} \mathscr{U})}) \subset \mathrm{st}(T, \mathrm{st} \mathscr{V})$.

Proof. Take any $x \in \overline{\mathrm{st}(S, \mathrm{st} \mathscr{U})}$. Since $\mathscr{U}$ is an open covering of $X$, there exists a $U_{1} \in \mathscr{U}$ such that

$$
x \in U_{1} .
$$

Since $x \in \overline{\operatorname{st}(S, \text { st } \mathscr{U})}$, then $U_{1} \cap \mathbf{s t}(S$, st $\mathscr{U}) \neq \varnothing$, i.e., there exists an $x_{1} \in$ $U_{1} \cap \operatorname{st}(S$, st $\mathscr{U})$. Then, there exists a $U_{2} \in \mathscr{U}$ such that

$$
x_{1} \in \mathbf{s t}\left(U_{2}, \mathscr{U}\right)
$$

and

$$
S \cap \operatorname{st}\left(U_{2}, \mathscr{U}\right) \neq \varnothing .
$$

By (3), there exists an $x_{2} \in S \cap \operatorname{st}\left(U_{2}, \mathscr{U}\right)$. Since $f^{-1 \mathscr{V}}>$ st $\mathscr{U}>\mathscr{U}$, there exist $V_{1}, V_{2} \in \mathscr{V}$ such that $f^{-1} V_{1} \supset U_{1}$ and $f^{-1} V_{2} \supset \operatorname{st}\left(U_{2}, \mathscr{U}\right)$. Then,

$$
V_{1} \supset f\left(U_{1}\right) \text { and } V_{2} \supset f\left(\operatorname{st}\left(U_{2}, \mathscr{U}\right)\right) \text {. }
$$

Since $f(S) \subset \operatorname{st}(T, \mathscr{V})$, then $f\left(x_{2}\right) \in \operatorname{st}(T, \mathscr{V})$. Thus, there exists a $V_{3} \in \mathscr{V}$ such that

$$
f\left(x_{2}\right) \in V_{3} \text { and } \quad V_{3} \cap T \neq \varnothing .
$$

By (1), (4), (2), and (5),

$$
f(x) \in \operatorname{st}\left(V_{2}, \mathscr{V}\right) \text { and } \operatorname{st}\left(V_{2}, \mathscr{V}\right) \cap T \neq \varnothing .
$$

This means that $f(x) \in \mathbf{s t}(T$, st $\mathscr{V})$. Hence, $f(\overline{\operatorname{st}(S, \text { st } \mathscr{U})}) \subset \operatorname{st}(T$, st $\mathscr{V})$.

(9.8) Lemma. Let $\mathscr{U}, \mathscr{V} \in \operatorname{Cov}(X)$.

(i) $\operatorname{st}(\langle\mathscr{V}\rangle)<\langle$ st $\mathscr{V}\rangle$.

(ii) If st $\mathscr{V}<\mathscr{U}$, then $\operatorname{st}(\langle\mathscr{V}\rangle)<\langle\mathscr{U}\rangle$. 
Proof. We show (i). Take any $\left\langle V_{1}, \ldots, V_{n}\right\rangle \in\langle\mathscr{V}\rangle$. We show that

$$
\operatorname{st}\left(\left\langle V_{1}, \ldots, V_{n}\right\rangle,\langle\mathscr{V}\rangle\right) \subset\left\langle W_{1}, \ldots, W_{n}\right\rangle .
$$

Here $W_{i}=\operatorname{st}\left(V_{i}, \mathscr{V}\right), i=1,2, \ldots, n$. Take any $K \in \operatorname{st}\left(\left\langle V_{1}, \ldots, V_{n}\right\rangle,\langle\mathscr{V}\rangle\right)$. Thus, there exists an $\left\langle V_{1}^{\prime}, \ldots, V_{m}^{\prime}\right\rangle \in\langle\mathscr{V}\rangle$ such that

$$
K \in\left\langle V_{1}^{\prime}, \ldots, V_{m}^{\prime}\right\rangle
$$

and

$$
\left\langle V_{1}, V_{2}, \ldots, V_{n}\right\rangle \cap\left\langle V_{1}^{\prime}, V_{2}^{\prime}, \ldots, V_{m}^{\prime}\right\rangle \neq \varnothing .
$$

By (3), there exists an $L \in\left\langle V_{1}, V_{2}, \ldots, V_{n}\right\rangle \cap\left\langle V_{1}^{\prime}, V_{2}^{\prime}, \ldots, V_{m}^{\prime}\right\rangle$. Thus,

$$
L \subset V_{1}^{\prime} \cup \cdots \cup V_{m}^{\prime}
$$

$$
L \cap V_{j}^{\prime} \neq \varnothing \text { for } j, 1 \leq j \leq m
$$

and

$$
L \subset V_{1} \cup \cdots \cup V_{n}
$$

$$
L \cap V_{i} \neq \varnothing \text { for } i, 1 \leq i \leq n \text {. }
$$

Take any $i, 1 \leq i \leq n$. By (4) and (7), there exists a $z_{i} \in L \cap V_{i} \subset L \subset V_{1}^{\prime} \cup$ $\cdots \cup V_{m}^{\prime}$. There is a $j(i), 1 \leq j(i) \leq m$, such that $z_{i} \in V_{j(i)}^{\prime}$, i.e., $V_{i} \cap V_{j(i)}^{\prime} \neq \varnothing$, in other words,

$$
V_{j(i)}^{\prime} \subset \operatorname{st}\left(V_{i}, \mathscr{V}\right)=W_{i} \text { for each } i, 1 \leq i \leq n .
$$

By (2),

$$
K \subset V_{1}^{\prime} \cup V_{2}^{\prime} \cup \cdots \cup V_{m}^{\prime}
$$

$$
K \cap V_{j}^{\prime} \neq \varnothing \text { for } j=1,2, \ldots, m \text {. }
$$

By (8) and (10), $\varnothing \neq K \cap V_{j(i)}^{\prime} \subset K \cap W_{i}$, i.e.,

$$
K \cap W_{i} \neq \varnothing \text { for each } i, 1 \leq i \leq n .
$$

Take any $j, 1 \leq j \leq m$. By (5) and (6), there exist $x_{j} \in L \cap V_{j}^{\prime} \subset L \subset$ $V_{1} \cup V_{2} \cup \cdots \cup V_{n}$. There exists an $i(j), 1 \leq i(j) \leq n$, such that $x_{j} \in V_{i(j)}$, i.e., $V_{j}^{\prime} \cap V_{i(j)} \neq \varnothing$. Thus,

$$
V_{j}^{\prime} \subset \operatorname{st}\left(V_{i(j)}, \mathscr{V}\right)=W_{i(j)} \quad \text { for each } j, 1 \leq j \leq m .
$$

By (9) and (12),

$$
K \subset W_{1} \cup \cdots \cup W_{n} .
$$

Thus, by (11) and (13), $K \in\left\langle W_{1}, W_{2}, \ldots, W_{n}\right\rangle$. Hence, we have (1).

Since $W_{i}=\operatorname{st}\left(V_{i}, \mathscr{V}\right) \in \operatorname{st} \mathscr{V},(1)$ means that $\operatorname{st}(\langle\mathscr{V}\rangle)<\langle$ st $\mathscr{V}\rangle$. Hence we have (i).

We show (ii). By (i), we have $\operatorname{st}(\langle\mathscr{V}\rangle)<\langle$ st $\mathscr{V}\rangle\langle\langle\mathscr{U}\rangle$. Hence we have (ii). 
(9.9) Lemma. If $\mathbf{p}: X \rightarrow(\mathscr{X}, \mathscr{U})$ is an approximate POL/ANR-resolution, then $\overline{\mathbf{p}}: 2^{X} \rightarrow 2^{(\mathscr{X}, \mathscr{U})}$ and $\overline{\mathbf{p}}: C(X) \rightarrow C(\mathscr{X}, \mathscr{U})$ are ANR-resolutions.

Proof. First, we consider $\overline{\mathbf{p}}: 2^{X} \rightarrow 2^{(\mathscr{X}, \mathscr{U})}$. Since $\mathbf{p}: X \rightarrow(\mathscr{X}, \mathscr{U})$ is an approximate resolution, it is an approximate map. By Lemma $(9.5), \overline{\mathbf{p}}: 2^{X} \rightarrow$ $2^{(\mathscr{X}, \mathscr{U})}$ is an approximate map. Thus, by $(1.12)$, it is sufficient to show conditions (B1) and (B2) hold for $\overline{\mathbf{p}}$.

We first show that $(\mathrm{B} 1)$ holds for $\overline{\mathbf{p}}$. Since $\mathbf{p}: X \rightarrow(\mathscr{X}, \mathscr{U})$ is an approximate resolution, by (1.12), p satisfies (B1) and (B2). Take any $\mathscr{U} \in \operatorname{Cov}\left(2^{X}\right)$. By Lemma (9.1), there exists a $\mathscr{V} \in \operatorname{Cov}(X)$ such that

$$
\langle\mathscr{V}\rangle<\mathscr{U} \text {. }
$$

By $(\mathrm{B} 1)$ for $\mathbf{p}: X \rightarrow(\mathscr{X}, \mathscr{U})$, there exists an $a \in A$ such that

$$
p_{a^{\prime}}^{-1} \mathscr{U}_{a^{\prime}}<\mathscr{V} \text { for any } a^{\prime}>a \text {. }
$$

By (2), Lemma (9.6), and (1),

$$
\left(\bar{p}_{a^{\prime}}\right)^{-1}\left(\left\langle\mathscr{U}_{a^{\prime}}\right\rangle\right)<\langle\mathscr{V}\rangle<\mathscr{U} \text { for any } a^{\prime}>a .
$$

This means that $\overline{\mathbf{p}}: 2^{X} \rightarrow 2^{(\mathscr{L}, \mathscr{U})}$ satisfies $(\mathrm{B} 1)$.

We now show that (B2) holds for $\overline{\mathbf{p}}$. We assume that $\overline{\mathbf{p}}$ does not satisfy $(\mathrm{B} 2)^{*}$ (since by (4.10) of [MW], (B2) and (B2)* are equivalent). Thus, there exists an $a_{0} \in A$ satisfying the following condition: For any $a^{\prime}>a_{0}$, there is a $\beta\left(a^{\prime}\right)>a^{\prime}$ such that

$$
\bar{p}_{a_{0} \beta\left(a^{\prime}\right)}\left(2^{X_{\beta\left(a^{\prime}\right)}}\right) \not \subset \mathrm{st}\left(\bar{p}_{a_{0}}\left(2^{X}\right), \mathrm{st}^{3}\left\langle\mathscr{U}_{a_{0}}\right\rangle\right) .
$$

By (4), there exists a $K_{\beta\left(a^{\prime}\right)} \in 2^{X_{\beta\left(a^{\prime}\right)}}$ such that

$$
\bar{p}_{a_{0} \beta\left(a^{\prime}\right)}\left(K_{\beta\left(a^{\prime}\right)}\right)=p_{a_{0} \beta\left(a^{\prime}\right)}\left(K_{\beta\left(a^{\prime}\right)}\right) \notin \operatorname{st}\left(\bar{p}_{a_{0}}\left(2^{X}\right), \mathrm{st}^{3}\left\langle\mathscr{U}_{a_{0}}\right\rangle\right) .
$$

We put $S_{a^{\prime}}=\left\{\bar{p}_{a^{\prime} \beta\left(a^{\prime \prime}\right)}\left(K_{\beta\left(a^{\prime \prime}\right)}\right): a^{\prime \prime} \in A\right.$ with $\left.a^{\prime \prime}>a^{\prime}\right\}$ for $a^{\prime}>a_{0}$. We show that

$$
\bar{p}_{a_{1} a_{2}}\left(S_{a_{2}}\right) \subset \operatorname{st}\left(S_{a_{1}},\left\langle\mathscr{U}_{a_{1}}\right\rangle\right) \text { for } a_{2}>a_{1}>a_{0} .
$$

To do so, take any $a^{\prime \prime}>a_{2}$. Thus, $\beta\left(a^{\prime \prime}\right)>a^{\prime \prime}>a_{2}>a_{1}$. Since $2^{(\mathscr{X}, \mathscr{C})}$ satisfies (A1) by Lemma (9.4), $\left(\bar{p}_{a_{1} a_{2}} \bar{p}_{a_{2} \beta\left(a^{\prime \prime}\right)}, \bar{p}_{a_{1} \beta\left(a^{\prime \prime}\right)}\right)<\left\langle\mathscr{U}_{a_{1}}\right\rangle$. Thus

$$
\bar{p}_{a_{1} a_{2}}\left(\bar{p}_{a_{2} \beta\left(a^{\prime \prime}\right)}\left(K_{\beta\left(a^{\prime \prime}\right)}\right)\right), \bar{p}_{a_{1} \beta\left(a^{\prime \prime}\right)}\left(K_{\beta\left(a^{\prime \prime}\right)}\right) \in\left\langle U_{1}, U_{2}, \ldots, U_{n}\right\rangle
$$

for some $\left\langle U_{1}, U_{2}, \ldots, U_{n}\right\rangle \in\left\langle\mathscr{U}_{a_{1}}\right\rangle$. Therefore,

$$
\bar{p}_{a_{1} a_{2}}\left(\bar{p}_{a_{2} \beta\left(a^{\prime \prime}\right)}\left(K_{\beta\left(a^{\prime \prime}\right)}\right)\right) \in \operatorname{st}\left(\bar{p}_{a_{1} \beta\left(a^{\prime \prime}\right)}\left(K_{\beta\left(a^{\prime \prime}\right)}\right),\left\langle\mathscr{U}_{a_{1}}\right\rangle\right) \subset \mathbf{s t}\left(S_{a_{1}},\left\langle\mathscr{U}_{a_{1}}\right\rangle\right) .
$$

Hence, we have (6).

Let $A^{\prime}=\left\{a \in A: a>a_{0}\right\}$. Since $A^{\prime}$ is cofinal in $A, \mathbf{p}^{\prime}=\left\{p_{a}: a \in A^{\prime}\right\}$ : $X \rightarrow(\mathscr{X}, \mathscr{U})^{\prime}=\left\{X_{a}, \mathscr{U}_{a}, p_{a a^{\prime}}, A^{\prime}\right\}$ is an approximate resolution of $X$. Thus we may assume that $A=A^{\prime}$, i.e., $A$ has a minimum index $a_{0}$ satisfying (4).

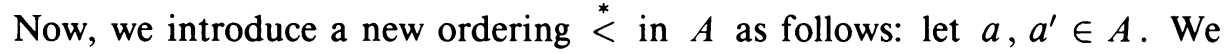
put $a \stackrel{*}{<} a^{\prime}$ provided (i) $a=a^{\prime}$ or (ii) $a<a^{\prime}, a \neq a^{\prime}$, and

$$
p_{a a^{\prime \prime}}^{-1} \mathscr{U}_{a}>\text { st } \mathscr{U}_{a^{\prime \prime}} \text { for any } a^{\prime \prime}>a^{\prime} .
$$


Note by the definition of $\stackrel{*}{<}^{*}$

$$
a \stackrel{*}{<} a^{\prime}, a \neq a^{\prime} \text {, and } a^{\prime}<a^{\prime \prime} \text { imply } a \stackrel{*}{<} a^{\prime \prime},
$$

and by $(\mathrm{A} 3)$

for each $a \in A$, there exists an $a^{\prime} \in A$ such that $a \stackrel{*}{<} a^{\prime}$.

Put $A^{*}=(A, \stackrel{*}{<})$. By $(8)$, it is easy to show that

$$
\mathbf{p}^{*}=\left\{p_{a}: a \in A\right\}: X \rightarrow(\mathscr{X}, \mathscr{U})^{*}=\left\{X_{a}, \mathscr{U}_{a}, p_{a a^{\prime}}, A^{*}\right\}
$$

is an approximate resolution.

Let $a \stackrel{*}{<} a^{\prime}$. Since $p_{a a^{\prime \prime}}^{-1} \mathscr{U}_{a}>$ st $\mathscr{U}_{a^{\prime \prime}}$ by (7), Lemmas (9.2) and (9.8) imply that $\left(\bar{p}_{a a^{\prime \prime}}\right)^{-1}\left\langle\mathscr{U}_{a}\right\rangle>\left\langle\right.$ st $\left.\mathscr{U}_{a^{\prime \prime}}\right\rangle>\mathbf{s t}\left\langle\mathscr{U}_{a^{\prime \prime}}\right\rangle$, i.e.,

$$
\left(\bar{p}_{a a^{\prime \prime}}\right)^{-1}\left(\left\langle\mathscr{U}_{a}\right\rangle\right)>\operatorname{st}\left\langle\mathscr{U}_{a^{\prime \prime}}\right\rangle \text { for any } a^{\prime \prime}>^{*} a^{\prime} .
$$

By (6), (11), and Lemma (9.7),

$$
\bar{p}_{a a^{\prime}}\left(\overline{\mathbf{s t}\left(S_{a^{\prime}}, \mathbf{s t}\left(\left\langle\mathscr{U}_{a^{\prime}}\right\rangle\right)\right)}\right) \subset \overline{\mathbf{s t}\left(S_{a}, \mathbf{s t}\left(\left\langle\mathscr{U}_{a}\right\rangle\right)\right)} \text { for } a^{*}<a^{\prime} .
$$

Since $S_{a} \neq \varnothing, T_{a}=\overline{\operatorname{st}\left(S_{a}, \text { st }\left\langle\mathscr{U}_{a}\right\rangle\right)}$ is a nonempty compact Hausdorff space for each $a \in A^{*}$. We show that

$$
\mathscr{T}=\left\{T_{a},\left\langle\mathscr{U}_{a}\right\rangle\left|T_{a}, p_{a a^{\prime}}\right| T_{a^{\prime}}, A^{*}\right\}
$$

is an approximate inverse system of nonempty compact Hausdorff spaces.

By (12), $\bar{p}_{a a^{\prime}}\left(T_{a^{\prime}}\right) \subset T_{a}$ for any $a \stackrel{*}{*} a^{\prime}$. Thus $\mathscr{T}$ is well defined. We need to show (A1)-(A3) for $\mathscr{T}$.

Since $(\mathscr{Z}, \mathscr{U})^{*}$ is an approximate inverse system, so is $2^{(\mathscr{X}, \mathscr{U})^{*}}$. Then $2^{(\mathscr{C}, \mathscr{C})^{*}}$ satisfies $(\mathrm{A} 1)-(\mathrm{A} 3)$. (A1) for $\mathscr{T}$ follows from $(\mathrm{A} 1)$ for $2^{(\mathscr{C}, \mathscr{C})^{*}}$.

To prove (A2) for $\mathscr{T}$, take any $\mathscr{V} \in \operatorname{Cov}\left(T_{a}\right)$ for any $a \in A$. Let $\mathscr{V}=$ $\left\{V_{1}, \ldots, V_{n}\right\}$. Then there are open subsets $U_{i} \subset 2^{X_{a}}$ such that

$$
U_{i} \cap T_{a}=V_{i} \text { for } i=1,2, \ldots, n .
$$

Since $T_{a}$ is closed in $2^{X_{a}}, \mathscr{U}=\left\{U_{1}, U_{2}, \ldots, U_{n}\right\} \cup\left\{2^{X_{a}}-T_{a}\right\}$ is an open covering of $2^{X_{a}}$. By (A2) for $2^{(\mathscr{X}, \mathscr{U})^{*}}$, there exists an $a^{\prime}>^{*} a$ such that

$$
\left(\bar{p}_{a a_{1}}\left|T_{a_{1}} \bar{p}_{a_{1} a_{2}}\right| T_{a_{2}}, \bar{p}_{a a_{2}} \mid T_{a_{2}}\right)<\mathscr{U} \text { for } a_{2} \stackrel{*}{>} a_{1} \stackrel{*}{>} a^{\prime} .
$$

Since $\bar{p}_{a a^{\prime}}\left(T_{a^{\prime}}\right) \subset T_{a}$ for $a^{\prime}{ }^{*} a,(15)$ means that $\left(\bar{p}_{a a_{1}}\left|T_{a_{1}} \bar{p}_{a_{1} a_{2}}\right| T_{a_{2}}, \bar{p}_{a a_{2}} \mid T_{a_{2}}\right)<$ $\mathscr{V}$ for $a_{2} \stackrel{*}{*}^{*} a_{1}>^{*} a^{\prime}$ in $\mathscr{T}$. Thus, we have (A2) for $\mathscr{T}$.

To prove (A3) for $\mathscr{T}$, take any $\mathscr{V} \in \operatorname{Cov}\left(T_{a}\right)$ for any $a \in A$. By the same way as in (A2) for $\mathscr{T}$, we define an open covering $\mathscr{U} \in \operatorname{Cov}\left(2^{X_{a}}\right)$. By (A3) for $2^{(\mathscr{X}, \mathscr{U})^{*}}$, there exists an $a^{\prime}>a$ such that

$$
\left(\bar{p}_{a a^{\prime \prime}}\right)^{-1} \mathscr{U}>\left\langle\mathscr{U}_{a^{\prime \prime}}\right\rangle \text { for } a^{\prime \prime}>^{*} a^{\prime} .
$$

Since $\bar{p}_{a a^{\prime \prime}}\left(T_{a^{\prime \prime}}\right) \subset T_{a}$ for $a^{\prime \prime}>^{*} a^{\prime}$ and $\mathscr{U} \mid T_{a}=\left\{U \cap T_{a}: U \in \mathscr{U}\right\}=\mathscr{V},(16)$ implies that

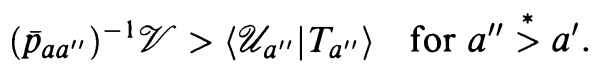


This means that $\mathscr{T}$ satisfies (A3). Hence $\mathscr{T}$ is an approximate inverse system, i.e., we have (13).

Since $\mathscr{T}$ is an approximate inverse system of nonempty compact Hausdorff spaces $T_{a}$, by (4.1) of [MW] $\lim \mathscr{T} \neq \varnothing$. Let $\mathbf{t}=\left\{t_{a}: a \in A^{*}\right\}: \lim \mathscr{T} \rightarrow \mathscr{T}$ be an approximate limit. Take any $M \in \lim \mathscr{T}$ and put $M_{a}=t_{a}(M)$ for $a \in A^{*}$. Thus,

$$
M_{a}=\lim \left\{\bar{p}_{a a^{\prime}}\left(M_{a^{\prime}}\right): a^{\prime} \in A^{*} \text { with } a^{\prime}>^{*} a\right\}
$$

in $T_{a}$ for each $a \in A^{*}$. That is, for each $a \in A^{*}$ and each $\mathscr{U} \in \operatorname{Cov}\left(2^{X_{a}}\right)$ there exists a $\gamma(a, \mathscr{U}) \in A^{*}$ such that $\gamma(a, \mathscr{U}) \stackrel{*}{>} a$ and

$$
\bar{p}_{a a^{\prime}}\left(M_{a^{\prime}}\right) \in \operatorname{st}\left(M_{a}, \mathscr{U}\right) \text { for } a^{\prime} \stackrel{>}{*}^{\prime} \gamma(a, \mathscr{U}) .
$$

Let $\beta(a)=\gamma\left(a,\left\langle\mathscr{U}_{a}\right\rangle\right)$ for each $a \in A^{*}$. We show that

$$
p_{a a^{\prime}}\left(M_{a^{\prime}}\right) \subset \operatorname{st}\left(M_{a}, \mathscr{U}_{a}\right) \text { for } a^{\prime} \stackrel{>}{>}^{*} \beta(a) .
$$

By the choice of $a^{\prime}, \bar{p}_{a a^{\prime}}\left(M_{a^{\prime}}\right) \in \operatorname{st}\left(M_{a},\left\langle\mathscr{U}_{a}\right\rangle\right)$. Thus, there exists $\left\langle U_{1}, U_{2}\right.$, $\left.\ldots, U_{n}\right\rangle \in\left\langle\mathscr{U}_{a}\right\rangle$ such that

$$
\bar{p}_{a a^{\prime}}\left(M_{a^{\prime}}\right), M_{a} \in\left\langle U_{1}, U_{2}, \ldots, U_{n}\right\rangle .
$$

By (21),

$$
\begin{gathered}
p_{a a^{\prime}}\left(M_{a^{\prime}}\right) \cup M_{a} \subset U_{1} \cup U_{2} \cup \cdots \cup U_{n}, \\
M_{a} \cap U_{i} \neq \varnothing \quad \text { for } i, 1 \leq i \leq n, \\
p_{a a^{\prime}}\left(M_{a^{\prime}}\right) \cap U_{i} \neq \varnothing \quad \text { for } i, 1 \leq i \leq n .
\end{gathered}
$$

By (23), $U_{1} \cup \cdots \cup U_{n} \subset \operatorname{st}\left(M_{a}, \mathscr{U}\right)$. Thus by (22), $p_{a a^{\prime}}\left(M_{a^{\prime}}\right) \subset U_{1} \cup \cdots \cup U_{n} \subset$ st $\left(M_{a}, \mathscr{U}\right)$, i.e., we have (20).

We introduce an order $\stackrel{* *}{<}$ in $A$ as follows: $a \stackrel{* *}{<} a^{\prime}$ provided (i) $a=a^{\prime}$ or (ii) $a \neq a^{\prime}, \beta(a)<a^{\prime}$.

Note that

$$
a \stackrel{* *}{<} a^{\prime}, a \neq a^{\prime}, \text { and } a^{\prime}<^{*} a^{\prime \prime} \text { imply } \quad a \stackrel{* *}{<} a^{\prime \prime},
$$

(26) for each $a \in A$, there exists an $a^{\prime} \in A$ such that $a \stackrel{* *}{<} a^{\prime}, a \neq a^{\prime}$.

Put $A^{* *}=(A, \stackrel{* *}{<})$. By $(25)$ it is easy to show that

$$
\mathbf{p}^{* *}=\left\{p_{a}: a \in A\right\}: X \rightarrow(\mathscr{X}, \mathscr{U})^{* *}=\left\{X_{a}, \mathscr{U}_{a}, p_{a a^{\prime}}, A^{* *}\right\}
$$

is an approximate resolution.

Since $a \stackrel{* *}{<} a^{\prime}$ implies $a \stackrel{*}{<} a^{\prime}$ by the definition,

$$
p_{a a^{\prime \prime}}^{-1} \mathscr{U}_{a}>\mathrm{st} \mathscr{U}_{a^{\prime \prime}} \text { for } a^{\prime \prime}{ }^{* *} a^{\prime} .
$$

By (20), (28), and Lemma (9.7),

$$
p_{a a^{\prime}}\left(\overline{\mathbf{s t}\left(M_{a^{\prime}}, \mathbf{s t} \mathscr{U}_{a^{\prime}}\right)}\right) \subset \overline{\mathbf{s t}\left(M_{a}, \mathbf{s t} \mathscr{U}_{a}\right)} \text { for } a^{\prime} \stackrel{* *}{>} a .
$$

By $(29),(\mathscr{L}, \mathscr{U} \mid L)=\left\{L_{a}, \mathscr{U}_{a}\left|L_{a}, p_{a a^{\prime}}\right| L_{a^{\prime}}, A^{* *}\right\}$ is an approximate inverse system. Here $L_{a}=\overline{\operatorname{st}\left(M_{a}, \text { st } \mathscr{U}_{a}\right)}$. 
Let $\pi=\left\{\pi_{a}: a \in A\right\}: \lim \mathscr{X} \rightarrow \mathscr{X}$ and $\pi^{\prime}=\left\{\pi_{a}^{\prime}: a \in A\right\}: \lim \mathscr{L} \rightarrow \mathscr{L}$ be the canonical approximate inverse limits. By definition, $\Pi\left\{L_{a}: a \in A\right\} \supset$ $\lim \mathscr{L} \subset \lim \mathscr{X} \subset \Pi\left\{X_{a}: a \in A\right\}$ and $\pi_{a}^{\prime}=\pi_{a} \mid \lim \mathscr{L} . \pi_{a}$ is induced by the projections $\pi_{a}: \Pi X_{a} \rightarrow X_{a}$.

Since $\mathbf{p}: X \rightarrow(\mathscr{X}, \mathscr{U})$ is a resolution of compact spaces, by (9.1) of [MW] it is a limit. Thus there exists a homeomorphism $f: \lim \mathscr{X} \rightarrow X$ such that

$$
p_{a} f=\pi_{a} \text { for each } a \in A .
$$

Now let $L=f(\lim \mathscr{L}) \subset X$. Since $\lim \mathscr{L}$ is compact by $(5.1)$ of [MW], $L$ is also compact, i.e.,

$$
L \in 2^{X} \text {. }
$$

By (30), $\mathbf{p} \mid L=\left\{p_{a} \mid L: a \in A\right\}: L \rightarrow(\mathscr{L}, \mathscr{U} \mid \mathscr{L})$ is also an approximate inverse limit. By (4.2) of [MW],

$$
\mathbf{p} \mid L: L \rightarrow(\mathscr{L}, \mathscr{U} \mid \mathscr{L}) \text { is an approximate resolution. }
$$

By (B2) for $\mathbf{p} \mid L$, there exists an $a_{1}>a_{0}$ such that

$$
p_{a_{0} a^{\prime \prime}}\left(L_{a^{\prime \prime}}\right) \subset \operatorname{st}\left(p_{a_{0}}(L), \mathscr{U}_{a_{0}}\right) \text { for any } a^{\prime \prime}>a_{1} \text {. }
$$

Let $\mathscr{U}=\left\{U_{1}, \ldots, U_{m}\right\} \in \operatorname{Cov}\left(X_{a_{0}}\right)$ and put $\left\{U_{1}, \ldots, U_{n}\right\}=\left\{U_{i} \in \mathscr{U}\right.$ : $\left.U_{i} \cap p_{a_{0}} L \neq \varnothing\right\}$. We show that

$$
\text { there exists } a_{2}>a_{1} \text { such that }
$$

$$
p_{a_{0}}(L), p_{a_{0} a^{\prime \prime}}\left(L_{a^{\prime \prime}}\right) \in\left\langle U_{1}, \ldots, U_{n}\right\rangle \text { for any } a^{\prime \prime}>a_{2} \text {. }
$$

Take any $i, 1 \leq i \leq n$. Since $U_{i} \cap p_{a_{0}}(L) \neq \varnothing$, there exists an $x=$ $\left(x_{a^{\prime \prime}}\right) \in L$ such that $p_{a_{0}}(x)=x_{a_{0}} \in U_{i}$. Since $x \in L=\lim \mathscr{L}, x_{a}=$ $\lim _{a^{\prime \prime}>a_{0}} p_{a_{0} a^{\prime \prime}}\left(x_{a^{\prime \prime}}\right)$. Thus, there exists an $a_{2}^{i}>a_{1}$ such that

$$
p_{a a^{\prime \prime}}\left(x_{a^{\prime \prime}}\right) \in U_{i} \quad \text { for any } a^{\prime \prime}>a_{2}^{i} \text {. }
$$

Take any $a_{2}>a_{2}^{1}, a_{2}^{2}, \ldots, a_{2}^{n}$. Take any $a^{\prime \prime}>a_{2}$. By (35),

$$
U_{i} \cap p_{a_{0} a^{\prime \prime}}\left(L_{a^{\prime \prime}}\right) \neq \varnothing \quad \text { for any } i=1,2, \ldots, n \text {. }
$$

Since $p_{a_{0} a^{\prime \prime}}\left(L_{a^{\prime \prime}}\right) \subset \operatorname{st}\left(p_{a_{0}}(L), \mathscr{U}_{a_{0}}\right)=U_{1} \cup \cdots \cup U_{n}$ by (33), (36) means that

$$
p_{a_{0} a^{\prime \prime}}\left(L_{a^{\prime \prime}}\right), p_{a_{0}}(L) \in\left\langle U_{1}, \ldots, U_{n}\right\rangle \text { for any } a^{\prime \prime}>a_{2} .
$$

Thus (37) implies (34). Hence, (34) means that

$$
\lim _{a^{\prime \prime}>a_{0}} p_{a_{0} a^{\prime \prime}}\left(L_{a^{\prime \prime}}\right)=p_{a_{0}}(L) \text { in } 2^{X_{a_{0}}}
$$

Next, we show that

$$
\lim _{a^{\prime \prime}>a_{0}} p_{a_{0} a^{\prime \prime}}\left(L_{a^{\prime \prime}}\right)=M_{a_{0}} \text { in } 2^{X_{a_{0}}} .
$$

Take any $\mathscr{U}, \mathscr{V} \in \operatorname{Cov}\left(X_{a_{0}}\right)$ with st $\mathscr{V}<\mathscr{U}$. By Lemma (9.8), st $\langle\mathscr{V}\rangle<$ $\langle\mathscr{U}\rangle$. Take any $\left\langle V_{1}, \ldots, V_{n}\right\rangle \in\langle\mathscr{V}\rangle$ such that $M_{a_{0}} \in\left\langle V_{1}, \ldots, V_{n}\right\rangle$. By the definition of $M_{a_{0}}, \lim _{a^{\prime \prime}>a_{0}} p_{a_{0} a^{\prime \prime}}\left(M_{a^{\prime \prime}}\right)=M_{a_{0}}$. Thus, there exist an $a_{1}>a_{0}$ such that for any $a^{\prime \prime}>a_{1}$

$$
p_{a_{0} a^{\prime \prime}}\left(M_{a^{\prime \prime}}\right) \in\left\langle V_{1}, \ldots, V_{n}\right\rangle
$$


By (A1), there exists an $a_{2}>a_{1}$ such that

$$
p_{a_{0} a^{\prime \prime}}^{-1} \mathscr{V}>\mathrm{st}^{2} \mathscr{U}_{a^{\prime \prime}} \text { for any } a^{\prime \prime}>a_{2} .
$$

Take any $a^{\prime \prime}>a_{2}$ and put $\operatorname{st}\left(M_{a^{\prime \prime}}\right.$, st $\left.\mathscr{U}_{a^{\prime \prime}}\right)=U_{1} \cup \cdots \cup U_{s}$, i.e., $U_{j} \in$ st $\mathscr{U}_{a^{\prime \prime}}, U_{j} \cap$ $M_{a^{\prime \prime}} \neq \varnothing$. Thus

$$
M_{a^{\prime \prime}} \subset \overline{\operatorname{st}\left(M_{a^{\prime \prime}}, \text { st } \mathscr{U}_{a^{\prime \prime}}\right)}=\bar{U}_{1} \cup \cdots \cup \bar{U}_{s}=L_{a^{\prime \prime}} .
$$

Since $\bar{U}_{j} \subset \operatorname{st}\left(U_{j}, \mathscr{U}_{a^{\prime \prime}}\right)$, by (42)

$$
\begin{aligned}
M_{a^{\prime \prime}}, L_{a^{\prime \prime}} & \in\left\langle\operatorname{st}\left(U_{1}, \text { st } \mathscr{U}_{a^{\prime \prime}}\right), \operatorname{st}\left(U_{2}, \text { st } \mathscr{U}_{a^{\prime \prime}}\right), \ldots, \operatorname{st}\left(U_{s}, \text { st } \mathscr{U}_{a^{\prime \prime}}\right)\right\rangle \\
& \in\left\langle\mathbf{s t}^{2} \mathscr{U}_{a^{\prime \prime}}\right\rangle .
\end{aligned}
$$

By (41) there exist $V_{1}^{\prime}, \ldots, V_{s}^{\prime} \in \mathscr{V}$ such that $p_{a_{0} a^{\prime \prime}}\left(\operatorname{st}\left(U_{j}\right.\right.$, st $\left.\left.\mathscr{U}_{a^{\prime \prime}}\right)\right) \subset V_{j}^{\prime}$ for $j=1,2, \ldots, s$. Then

$$
p_{a_{0} a^{\prime \prime}}\left(M_{a^{\prime \prime}}\right), p_{a_{0} a^{\prime \prime}}\left(L_{a^{\prime \prime}}\right) \in\left\langle V_{1}^{\prime}, \ldots, V_{s}^{\prime}\right\rangle \in\langle\mathscr{V}\rangle .
$$

By (40) and (44), $\left\langle V_{1}, \ldots, V_{n}\right\rangle \cap\left\langle V_{1}^{\prime}, \ldots, V_{s}^{\prime}\right\rangle \neq \varnothing$. Thus, since st $\langle\mathscr{V}\rangle\langle\langle\mathscr{U}\rangle$

$$
p_{a_{0} a^{\prime \prime}}\left(L_{a^{\prime \prime}}\right) \in \operatorname{st}\left(M_{a_{0}}, \operatorname{st}\langle\mathscr{V}\rangle\right) \subset \operatorname{st}\left(M_{a_{0}},\langle\mathscr{U}\rangle\right) .
$$

This means (39).

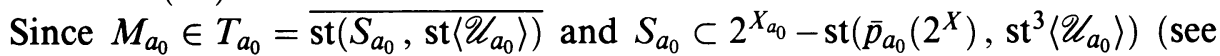
(5)), then $M_{a_{0}} \in 2^{X_{a_{0}}}-\operatorname{st}\left(\bar{p}_{a_{0}}\left(2^{X}\right),\left\langle\mathscr{U}_{a_{0}}\right\rangle\right)$, i.e.,

$$
M_{a_{0}} \notin \bar{p}_{a_{0}}\left(2^{X}\right) \text {. }
$$

By (38) and (39) $M_{a_{0}}=\lim p_{a_{0} a^{\prime \prime}}\left(L_{a^{\prime \prime}}\right)=p_{a_{0}}(L) \in \bar{p}_{a_{0}}\left(2^{X}\right)$, i.e., $M_{a_{0}} \in \bar{p}_{a_{0}}\left(2^{X}\right)$. This contradicts (45). Hence $\overline{\mathbf{p}}$ satisfies (B2). Hence $\overline{\mathbf{p}}: 2^{X} \rightarrow 2^{(\mathscr{X}, \mathscr{U})}$ is an approximate resolution. Since $X_{a}$ is a locally connected metric compactum, $2^{X_{a}}$ is an ANR and so $\overline{\mathbf{p}}: 2^{X} \rightarrow 2^{(\mathscr{X}, \mathscr{U})}$ is an ANR-resolution.

Next we must show that $\overline{\mathbf{p}}: C(X) \rightarrow C(\mathscr{X}, \mathscr{U})$ is an approximate resolution. When, in the previous proof, we replace $2^{X}$ and $2^{(\mathscr{Z}, \mathscr{U})}$ by $C(X)$ and $C(\mathscr{X}, \mathscr{U})$, respectively, we can use our previous proof for this purpose. We must show that conditions (B1) and (B2) hold. We can easily show (B1) as before. To show (B2) we must pay attention to connectedness in the previous proof of (B2). Since the $X_{a}$ are polyhedra or ANR's, they are also locally connected. Making use of the cofiniteness of the index set, we may assume that all coverings $\mathscr{U}_{a}$ consist of connected open sets. Therefore, in the proof of the condition (B2), since $M_{a} \in C\left(X_{a}\right)$, we have $L_{a}=\overline{\operatorname{st}\left(M_{a}, \text { st } \mathscr{U}_{a}\right)}$ are continua. Thus, by the following Lemma (9.10), $L=\lim \mathscr{L}$ is a continuum. In the same way as before, we obtain a contradiction. So we have (B2). Thus, $\overline{\mathbf{p}}: C(X) \rightarrow C(\mathscr{X}, \mathscr{U})$ is an approximate resolution. Hence we have completed the proof of (9.9).

(9.10) Lemma. Let $\mathbf{p}: X \rightarrow(\mathscr{X}, \mathscr{U})$ be an approximate resolution. Let $X$ and all $X_{a}$ be compact Hausdorff spaces. If all the $X_{a}$ are connected, so is $X$. Proof. We assume that $X$ is not connected. Then $X$ has at least two components, $X_{1}$ and $X_{2}$. Since $X$ is compact, components are quasi-components. Thus there is an open-closed subset $U$ of $X$ such that $X_{1} \subset U, X_{2} \subset X-U$. Let $\mathscr{U}=\{U, X-U\} \in \operatorname{Cov}(X)$. By (B1), there exists an $a_{0} \in A$ such that

$$
p_{a_{0}}^{-1} \mathscr{U}_{a_{0}}<\mathscr{U} \text {. }
$$


Put $\mathscr{U}_{a_{0}}=\left\{U_{\beta}: \beta \in B\right\}$. Put $B_{0}=\left\{\beta \in B: p_{a_{0}}^{-1} U_{\beta} \subset U\right\}$ and $B_{1}=\{\beta \in$ $\left.B, p_{a_{0}}^{-1} U_{\beta} \subset X-U\right\}$. Since the elements of $\mathscr{U}$ are disjoint, by (1) $B=B_{0} \cup B_{1}$ and $B_{0} \cap B_{1}=\varnothing$. Moreover, $W_{0}=\bigcup\left\{U_{\beta}: \beta \in B_{0}\right\}$ and $W_{1}=\bigcup\left\{U_{\beta}: \beta \in B_{1}\right\}$ are open in $X_{a_{0}}$ and $W_{0} \cup W_{1}=X_{a_{0}}, W_{0} \cap W_{1}=\varnothing$. Since $X_{1} \neq \varnothing$ and $X_{2} \neq \varnothing$, then $W_{0} \neq \varnothing$ and $W_{1} \neq \varnothing$. This is a contradiction, since we have obtained the disconnectedness of $X_{a_{0}}$. Hence $X$ must be connected.

By Theorem (5.13) we have

(9.11) Theorem. If $X$ is a Hausdorff continuum with an approximate resolution $\mathbf{p}: X \rightarrow(\mathscr{X}, \mathscr{U})$ such that the induced resolution $\overline{\mathbf{p}}: 2^{X} \rightarrow\left(2^{(\mathscr{X}, \mathscr{U})}\right)$ (or $\overline{\mathbf{p}}: C(X) \rightarrow C(\mathscr{X}, \mathscr{U}))$ is a cosmic approximate ANR-resolution, then $2^{X}(C(X))$ has the fixed point property.

(9.12) Corollary. If $X$ is an arc-like (or circle-like) Hausdorff continuum, then $C(X)$ has the fixed point property.

Proof. Since $X$ is arc-like (circle-like) there is an approximate resolution $\mathbf{p}$ : $X \rightarrow(\mathscr{X}, \mathscr{U})$ where all the coordinate spaces are arcs (circles) and the bonding maps are surjective. Then by an argument due to Rogers [R, Theorem 4] $\bar{p}_{a a^{\prime}}$ : $C\left(X_{a^{\prime}}\right) \rightarrow C\left(X_{a}\right)$ is a cosmic map between 2-cells. So $\overline{\mathbf{p}}: C(X) \rightarrow C(\mathscr{X}, \mathscr{U})$ is a cosmic approximate ANR-resolution. Therefore by $(9.11) C(X)$ has the fixed point property.

(9.13) Corollary. If $X$ is a locally connected Hausdorff continuum, then $2^{X}$ and $C(X)$ have the fixed point property.

Proof. We consider only $2^{X}$, the case of $C(X)$ is similar. By Theorem 2 of [M2] $X$ is the (inverse) limit of a monotone system of locally connected metric continua $\mathbb{X}$. We consider $\mathbb{X}$ as an approximate system

$$
\mathscr{X}=\left\{X_{a}, \mathscr{U}_{a}, p_{a a^{\prime}}, A\right\},
$$

where $p_{a a^{\prime}}: X_{a^{\prime}} \rightarrow X_{a}$ is a monotone surjective map of locally connected metric continua.

Then we have $\bar{p}_{a a^{\prime}}: 2^{X_{a^{\prime}}} \rightarrow 2^{X_{a}}$ is a cosmic map of ANR's by Theorem 2.3 of [N2]. Therefore $\overline{\mathbf{p}}: 2^{X} \rightarrow 2^{(\mathscr{X}, \mathscr{U})}$ is a cosmic approximate ANR-resolution and by (9.11), $2^{X}$ has the fixed point property.

Remark. The above corollary was known in the metric case since the hyperspaces are AR's in that case.

The following was obtained by Nadler in [N2] for the metric case.

(9.14) Corollary. Let $X$ have an approximate resolution $(\mathscr{X}, \mathscr{U})$ where each $X_{a}$ is a Peano continuum and for each $a \in A$ there is a subcontinuum $Y_{a^{\prime}}$ of $X_{a^{\prime}}$ for all $a^{\prime}>a$ such that $p_{a a^{\prime}} \mid Y_{a^{\prime}}: Y_{a^{\prime}} \rightarrow X_{a}$ maps $Y_{a^{\prime}}$ monotonically onto $X_{a}$. Then $2^{X}$ and $C(X)$ have the fixed point property.

Proof. We consider only $2^{X}$, since $C(X)$ is similar. By Theorem 2.3 of [N2] $\overline{\left(p_{a a^{\prime}} \mid Y_{a^{\prime}}\right)}: 2^{Y_{a}^{\prime}} \rightarrow 2^{X_{a}}$ is cosmic. Since $\overline{\left(p_{a a^{\prime}} \mid Y_{a^{\prime}}\right)}=\overline{p_{a a^{\prime}}} \mid 2^{Y_{a^{\prime}}}$, each $\bar{p}_{a a^{\prime}} \mid 2^{Y_{a^{\prime}}}:$ $2^{Y_{a^{\prime}}} \rightarrow 2^{X_{a}}$ is cosmic, hence so is $\overline{p_{a a^{\prime}}}: 2^{X_{a^{\prime}}} \rightarrow 2^{X_{a}}$ (see Proposition 5 of $[\mathrm{H}]$ ). Since each $X_{a}$ is a Peano continuum, each $2^{X_{a}}$ is an AR, so by Theorem (9.11) we have $2^{X}$ has the fixed point property. 


\section{REFERENCES}

[B] R. H. Bing, The elusive fixed property, Amer. Math. Monthly 76 (1969), 119-132.

[Bo1] K. Borsuk, Sur un continu acyclique qui se laisse transformer topologiquement en lui meme sans points invariants, Fund. Math. 24 (1935), 51-58.

[Bo2] _-, On nearly extendable maps, Bull. Acad. Polon. Sci. 23 (1975), 753-760.

[Bu] C. E. Burgess, Chainable continua and indecomposability, Pacific J. Math. 9 (1959), 653659.

[FR] J. Ford and J. W. Rogers, Refinable maps, Colloq. Math. 39 (1978), 263-269.

[H] W. Holsztynski, Universal mappings and fixed point theorems, Bull. Acad. Polon. Sci. 15 (1967), 433-438.

[HU] S. T. Hu, Theory of retracts, Wayne State Univ. Press, Detroit, Mich. 1965.

[K] A. Koyama, Proper refinable maps on locally compact paracompact spaces, Topology Proc. 8 (1983), 55-69.

[L] S. Lefschetz, Algebraic topology, Amer. Math. Soc. Colloq. Publ., vol. 27, Amer. Math. Soc., Providence, R.I., 1942.

[M] S. Mardešić, Chainable continua and inverse limits, Glasnik Math. 14 (1959), 219-232.

[M1] _ Approximate polyhedra, resolutions of maps and shape fibrations, Fund. Math. 114 (1981), 53-78.

[M2] _ Locally connected ordered and chainable continua, Rad. Jugoslav Akad. Znan. Umjet. Odjel Mat. Fiz. Tekn. Nauke 319 (1961), 147-166.

[MM] S. Mardešić and V. Matijević, $\mathscr{P}$-like spaces are limits of approximate $\mathscr{P}$-resolutions, Topology Appl. (to appear)

[MR] S. Mardešić and L. Rubin, Approximate inverse systems of compacta and covering dimension, Pacific J. Math. 138 (1989), 129-144.

[MS] S. Mardešić and J. Segal, Shape theory, North-Holland, Amsterdam, 1982.

[MS1] _, $\mathscr{P}$-like continua and approximate inverse limits, Math. Japon. 33 (1988), 895-908.

[MS2] _ Stability of almost commutative inverse systems of compacta, Topology Appl. 31 (1989), 285-299.

[MS3] _ Mapping approximate inverse systems, Fund. Math. 134 (1990), 73-91.

[MW] S. Mardešić and T. Watanabe, Approximate resolutions of spaces and mappings, Glasnik Math. 24 (1989), 587-637.

[Ma] M. M. Marsh, u-mappings on trees, Pacific J. Math. 127 (1987), 373-387.

[MiR] J. Mioduszewski and M. Rochowski, Remarks on fixed point theorem for inverse limit spaces, Colloq. Math. 9 (1962), 67-71.

[Mo] K. Morita, Completion of hyperspaces of compact subsets and topological completion of openclosed maps, General Topology Appl. 4 (1974), 217-233.

[N1] S. Nadler, Hyperspaces of sets, Marcel Dekker, New York, 1978.

[N2] _ Induced universal maps and some hyperspaces with the fixed point property, Proc. Amer. Math. Soc. 100 (1987), 749-754.

[R] J. T. Rogers, Hyperspaces of arc-like and circle-like continua, Topology Conf. (V.P.I., 1973), (R. F. Dickman and P. Fletcher, eds.), Lecture Notes in Math., vol. 375, Springer-Verlag, New York, 1974, pp. 231-235.

[S1] J. Segal, Hyperspaces of the inverse limit space, Proc. Amer. Math. Soc. 10 (1959), 706-709.

[S2] _ A fixed point theorem for the hyperspace of a snake-like continuum, Fund. Math. $\mathbf{5 0}$ (1961), 237-248.

[Sp] E. H. Spanier, Algebraic topology, McGraw-Hill, New York, 1966.

[W] T. Watanabe, Approximative shape theory, mimeographed notes, University of Yamaguchi, Yamaguchi, 1981-1984.

[W1] —, Approximate shape. I, Tsukuba J. Math. 11 (1987), 17-59.

[W2] _ Approximate shape. II, Tsukuba J. Math. 11 (1987), 303-339. 
[W3] _ Approximate shape. III, Tsukuba J. Math. 12 (1988), 1-41.

[W4] _ Shape classifications for complex projective space-like and wedges of $n$-spheres-like continua, Sci. Rep. Tokyo Kyoiku Daigaku Sect. A 12 (1974), 233-245.

Department of Mathematics, The University of Washington, Seattle, Washington 98195

E-mail address: segal@math.washington.edu

Department of Mathematics, University of Yamaguchi, Yamaguchi City, 753, Japan

E-mail address: F00300@sinet.ad.jp 